\title{
Recent Developments in Combinatorial Aspects of Normal Ordering
}

\author{
Matthias Schork \\ Im Haindell 99, 65843 Sulzbach, Germany \\ Email: MSchork1@gmx.de \\ Received: November 1, 2020, Accepted: February 7, 2021, Published: February 12, 2021 \\ The authors: Released under the CC BY-ND license (International 4.0)
}

ABSTRACT: In this paper, we report on recent progress concerning combinatorial aspects of normal ordering. After giving a short introduction to the history and motivation of normal ordering, we present some recent developments. Many of them are related to the quantum plane or the Weyl algebra or close relatives thereof. Along the way, a few open problems are mentioned.

2020 Mathematics Subject Classification: 05A05; 05A10; 05A15; 05A19; 05C20; 05C30; 11B73, 11B75; 13N15; 16S15; 16S32; 16T30

Keywords: Weyl algebra; Normal ordering; Stirling numbers; Bell numbers; Lah numbers; Whitney numbers; Dowling numbers; Dyck paths; Directed graphs; Rook numbers; Partitions; Quantum plane; Spivey identity; Binomial formula

\section{Introduction}

Maybe the first explicit results concerning normal ordering were derived by Scherk [120] in his dissertation from 1823. There he considered (in our terminology, see [22, Appendix A]) the two operators $X$ and $D$ acting on functions of a real variable by

$$
(X f)(x)=x f(x), \quad(D f)(x)=\frac{d f}{d x}(x) .
$$

These two operators do not commute, but have the commutation relation

$$
D X-X D=I,
$$

where $I$ denotes the identity. An arbitrary word in $X$ and $D$ can be written as $\omega=X^{r_{1}} D^{s_{1}} X^{r_{2}} D^{s_{2}} \cdots X^{r_{n}} D^{s_{n}}$ for some $r_{k}, s_{k} \in \mathbb{N}_{0}$. Since the operators do not commute one has to be careful to respect the order of the operators when acting on a function $f$. A word $\omega$ in the letters $X$ and $D$ is said to be in normal ordered form if $\omega=a_{r, s} X^{r} D^{s}$ for $r, s \in \mathbb{N}_{0}$ (and arbitrary coefficients $a_{r, s} \in \mathbb{C}$ ). An expression consisting of a sum of words is called normal ordered if each of the summands is normal ordered. The process of bringing a word - or a sum of words - into a normal ordered form (by using the commutation relation) is called normal ordering. For example, normal ordering the word $D^{2} X D$ yields $D X D^{2}+D^{2}$. Writing a word $\omega$ in its normal ordered form,

$$
\omega=\sum_{r, s \in \mathbb{N}_{0}} S_{r, s}(\omega) X^{r} D^{s},
$$

the uniquely determined coefficients $S_{r, s}(\omega)$ are called normal ordering coefficients. In our terminology, Scherk [120] determined the normal ordered form of the words $(X D)^{n}$ and $\left(X^{p} D\right)^{n}$, for $n \in \mathbb{N}$, and discussed combinatorial properties of the corresponding normal ordering coefficients. For example,

$$
(X D)^{n}=\sum_{k=1}^{n} S_{1,1}(n, k) X^{k} D^{k},
$$


where the coefficients satisfy the recurrence relation $S_{1,1}(n+1, k)=S_{1,1}(n, k-1)+k S_{1,1}(n, k)$. Thus, the normal ordering coefficients are precisely the Stirling numbers of the second kind $S(n, k)$, A008277 in [127], (which Scherk did not recognize) and one has a connection to set partitions. Presumably, (3) is the single most well-known result concerning normal ordering and has been discovered in different contexts again and again. As mentioned above, Scherk also discussed the analogous normal ordering coefficients $S_{p, 1}(n, k)$ - which are nowadays considered as a particular example of generalized Stirling numbers (for example, $S_{2,1}(n, k)=L(n, k)$, the unsigned Lah numbers, A105278 in [127]). In the same fashion, one may introduce the generalized Stirling numbers $S_{p, q}(n, k)$ by normal ordering $\left(X^{p} D^{q}\right)^{n}$, and again, this has been done several times in literature (e.g., by Carlitz, McCoy, Toscano - to mention just a few classical authors), see Mansour and Schork [98] for a comprehensive survey. Early normal ordering results were derived (and used) in an implicit fashion when special polynomials were considered with the methods of "operational calculus".

Recall that the (first) Weyl algebra $A_{1}$ can be defined by the two operators $X$ and $D$ satisfying (1). Thus, from this perspective, Scherk considered normal ordering in the Weyl algebra. More abstractly, we define the (first) Weyl algebra as the algebra generated by two symbols $U$ and $V$ satisfying the commutation relation $U V-V U=I$, where $I$ denotes the identity and we let $U^{0}=V^{0}=I$. Thus, $A_{1}=\mathbb{C}\langle U, V\rangle /(U V-V U-I)$.

A basic object in physical models of quantum theory is the creation operator $\hat{a}^{\dagger}$ which, roughly speaking, creates one quantum of a bosonic degree of freedom (in the single-mode case). One also has an analogous annihilation operator $\hat{a}$, and these two operators satisfy the bosonic commutation relation $\hat{a} \hat{a}^{\dagger}-\hat{a}^{\dagger} \hat{a}=I$, briefly $\left[\hat{a}, \hat{a}^{\dagger}\right]=I$. Thus, the algebra generated by $\hat{a}, \hat{a}^{\dagger}$ is $A_{1}$, and all algebraic relations carry over (in fact, in the physical application one has in addition an involution mapping $\hat{a}$ to $\hat{a}^{\dagger}$, and vice versa). In physical applications it is useful to write a word in these operators in such a fashion that all operators $\hat{a}$ stand to the right, i.e., in normal ordered form. Hence, the normal ordering problem was rediscovered in physics, and an explicit combinatorial connection was drawn by Katriel [77] in 1974 when he obtained the Stirling numbers of the second kind as normal ordering coefficients for $\left(\hat{a}^{\dagger} \hat{a}\right)^{n}$, see (3). This motivated physicists to consider the normal ordering problem for particular words in $\hat{a}, \hat{a}^{\dagger}$ and to introduce generalized Stirling numbers as normal ordering coefficients, thereby rediscovering many of the results mentioned above. When evaluating $\left(\hat{a}^{\dagger} \hat{a}\right)^{n}$ with respect to coherent states, Katriel [78] observed in 2000 that the Bell numbers $B_{n}$ (A000110 in [127]) and the Dobinski-formula appear. Again, this result has been generalized in different directions in the physical context, see [98]. The corresponding normal ordering problem for the creation and annihilation operators of a fermionic degree of freedom is not interesting: The corresponding operators $\hat{f}^{\dagger}$ and $\hat{f}$ satisfy the fermionic anti-commutation relation $\hat{f} \hat{f}^{\dagger}+\hat{f}^{\dagger} \hat{f}=I$, briefly $\left\{\hat{f}, \hat{f}^{\dagger}\right\}=I$, as well as $\hat{f}^{2}=0=\left(\hat{f}^{\dagger}\right)^{2}$. However, Navon [113] discovered in 1973 that the normal ordering coefficients for arbitrary words in the fermionic multi-mode case can be expressed as rook numbers. Let us mention that in addition to normal ordering other orderings are considered, e.g., anti-normal ordering (where all operators $\hat{a}$ stand to the left of all operators $\hat{a}^{\dagger}$ ) and symmetric ordering (Weyl ordering). Thus, the expression "reordering" can also be found in the literature to bring a word in non-commutative symbols (operators) into a particular ordered form.

Above, we described the Weyl algebra $A_{1}$ abstractly as being generated by symbols $U$ and $V$ satisfying the commutation relation $U V-V U=I$. By considering other relations for $U$ and $V$, one obtains other algebras for which one may consider the normal ordering problem. Let us give some examples.

Example 1.1 (Commuting symbols). If we let $U$ and $V$ commute, i.e., $U V=V U$, then the resulting structure is just $\mathbb{C}[U, V]$, the ring of polynomials in $U$ and $V$. The normal ordered form of a word $\omega$ containing $r$ letters $V$ and $s$ letters $U$ is just $\omega=V^{r} U^{s}$. More interesting is the binomial formula $(U+V)^{n}=\sum_{k=0}^{n}\left(\begin{array}{c}n \\ k\end{array}\right) V^{n-k} U^{k}$.

Example 1.2 (Quantum plane). Let $q$ be a variable commuting with all powers of $U$ and $V$. We will always assume that $q \in \mathbb{C}$ and to simplify computations we will further assume that $q$ is not a root of unity. If we let $U$ and $V$ q-commute, i.e., $U V=q V U$, then the resulting structure is called quantum plane. If we define for an arbitrary word $\omega=U^{r_{1}} V^{s_{1}} \cdots U^{r_{n}} V^{s_{n}}$ the quantities $R=r_{1}+\cdots+r_{n}, S=s_{1}+\cdots+s_{n}$, and $\mathcal{J}(\omega)=\sum_{k=1}^{n}\left(r_{k} \sum_{l=k}^{n} s_{l}\right)$, then the normal ordered form of $\omega$ is given by $\omega=q^{\mathcal{J}(\omega)} V^{S} U^{R}$. Note that $\mathcal{J}(\omega)$ counts the number of inversions. A famous result due to Potter (1950) and Schützenberger (1953) is the 
$q$-binomial formula,

$$
(U+V)^{n}=\sum_{k=0}^{n}\left(\begin{array}{l}
n \\
k
\end{array}\right)_{q} V^{n-k} U^{k},
$$

where the $q$-deformed binomial coefficient (or Gaussian coefficient) is given by $\left(\begin{array}{l}n \\ k\end{array}\right)_{q}=\frac{[n]_{q} !}{[k]_{q} ![n-k]_{q} !}$ and where the quantum numbers $[n]_{q}$ and their factorials are given by (see Cheung and Kac [38])

$$
[n]_{q}=1+q+q^{2}+\cdots+q^{n-1},[n]_{q} !=[n]_{q}[n-1]_{q}[n-2]_{q} \cdots[2]_{q}[1]_{q} .
$$

Recall that we assumed that $q$ is not a root of unity. However, one may consider what happens when $q$ approaches a root of unity. Clearly, for $q \rightarrow 1$, one returns to Example 1.1, while $q \rightarrow-1$ yields anti-commuting variables. Jackson [70] considered in 1910 the polynomials $(a+x)^{[n]}=(a+x)(a+x q) \cdots\left(a+x q^{n-1}\right)$ and showed

$$
(a+x)^{[n]}=\sum_{k=0}^{n}\left(\begin{array}{l}
n \\
k
\end{array}\right)_{q} q^{\left(\begin{array}{c}
k \\
2
\end{array}\right)} x^{k} a^{n-k}
$$

for the commuting variables a and $x$. Johnson [71] showed - following an argument of Andrews - that (4) and (6) are, in fact, equivalent.

Example 1.3 (q-deformed Weyl algebra). Similar as Example 1.2 provided a q-deformation of Example 1.1, one can consider a q-deformation of the Weyl algebra $A_{1}$ by letting the variables $U$ and $V$ satisfy a $q$-deformed variant of $U V-V U=I$. Thus, the $q$-deformed Weyl algebra $A_{1}(q)$ is generated by $U$ and $V$ satisfying

$$
U V-q V U=I
$$

Recall that $U \mapsto D$ and $V \mapsto X$ gives a concrete representation of the undeformed Weyl algebra. In a similar fashion, $U \mapsto D_{q}$ and $V \mapsto X$ gives a concrete representation of (7), where $D_{q}$ denotes the Jackson derivative

$$
\left(D_{q} f\right)(x)=\frac{f(x)-f(q x)}{(1-q) x},
$$

see [38]. Maybe the first explicit discussion of (7) in the physical context was by Frisch and Bourret [58] in 1970 who also considered normal ordering aspects. The commutation relation of the creation and annihilation operators of a $q$-deformed bosonic degree of freedom is given by $\hat{a}_{q} \hat{a}_{q}^{\dagger}-q \hat{a}_{q}^{\dagger} \hat{a}_{q}=I$, that is, exactly by (7). Katriel and Kibler [80] considered in 1992 normal ordering $\left(\hat{a}_{q}^{\dagger} \hat{a}_{q}\right)^{n}$ and discovered that the normal ordering coefficients are given by the q-deformed Stirling numbers of the second kind $S_{q}(n, k)$. From the mathematical side, Cigler [40] began in 1979 a deep study of the consequences of (7) and showed that the normal ordering coefficients of $(V U)^{n}$ are given by $S_{q}(n, k)$, thereby extending (3) to the q-deformed situation. Normal ordering other words in $U, V$ satisfying (7) was considered extensively in the mathematical and physical literature, see [65] and [98] for more results and references.

Example 1.4 (Other generalizations of the Weyl algebra). A generalization of the Weyl algebra was introduced by Varvak [131] in 2005 by considering the commutation relation

$$
U V-V U=h V^{s}
$$

where $s \in \mathbb{N}_{0}$ and $h \in \mathbb{C}$. She interpreted the normal ordering coefficients in the Weyl algebra $A_{1}$ as rook numbers, and gave a similar interpretation for variables satisfying (9). The particular case $s=2$, corresponding to the Jordan plane or meromorphic Weyl algebra had been considered in detail before (by Benaoum, Berry, Diaz and Pariguan, Shirikov and Viskov, see the extensive discussion in [98]). One particular result we would like to mention is the following result due to Benaoum [14] and Viskov [132]: Let $U$ and $V$ satisfy $U V-V U=h V^{2}$, then one has

$$
(U+V)^{n}=\sum_{k=0}^{n}\left(\begin{array}{l}
n \\
k
\end{array}\right)(1 \mid-h)_{k} V^{k} U^{n-k},
$$

where $(1 \mid-h)_{k}=\prod_{j=0}^{k-1}(1+h j)$. Benaoum [15] also considered the $q$-deformed situation and showed a $q$ analogue of (10). Returning to arbitrary s, the q-deformed variant $U V-q V U=h V^{s}$ of (9) was also studied and generalized Stirling numbers $\mathfrak{S}_{s ; h}(n, k \mid q)$ were defined by Mansour et al. [100, 101] as normal ordering coefficients of $(V U)^{n}$. Generalizing further, the relation $U V-q V U=h f(V)$ was considered by Mansour and Schork [97] as well as Benkart et al. [16, 17]. 
Above, we introduced those algebras which have been examined in most detail with respect to normal ordering (or to some other ordering scheme, like anti-normal ordering). Note that all structures considered above have only one nontrivial commutation relation. In this situation, one obtains nice combinatorial structures for the normal ordering coefficients. Clearly, one may similarly consider algebras having more generators as long as only two generators have a nontrivial commutation relation and the remaining generators are central. When considering algebras with three or more generators where none of the generators is central (hence, one has at least three nontrivial commutation relations) one can clearly define normal ordering in an analogous fashion but the considerations quickly become tedious. For example, motivated by the physical context discussed by Lohe and Thilagam [89], one may consider normal ordering words in $U, V, R$ satisfying

$$
U V-V U=I+\nu R, \quad U R=-R U, \quad R V=-V R, \quad R^{2}=I
$$

where $\nu$ is some real parameter. Other "mild extensions" of the commutation relation of the Weyl algebra have been considered in the physics literature. Another route to normal ordering in algebras having more than two generators is to consider the multi-mode case. Recall that although normal ordering in the single-mode case of the fermionic anti-commutation relation is not interesting from the combinatorial point of view, the multi-mode case involves rook numbers and is clearly interesting. In a similar fashion, one can consider the bosonic multi-mode case of the Weyl algebra, i.e., the $n$-th Weyl algebra $A_{n}$ (or a multi-mode generalization of some other structure mentioned above).

Before turning to describe the structure of the present article, we would like to give some comments on literature. A survey of the topics discussed here and a comprehensive bibliography up to the year 2015 can be found in Mansour and Schork [98]. The aim of the survey at hand is to present a guide to the mathematical literature for "recent" developments concerning normal ordering - which we interpret roughly as having appeared in 2015 or later. Thus, with great regret, we have chosen to mention from those papers having appeared before 2015 only the basic ones and refer for [98] for more references. For the recent developments, we will give complete references. The first survey concerning normal ordering from a combinatorial point of view is due to Blasiak and Flajolet [22] and appeared in 2011 - and it is still highly recommended. A host of reordering formulas (and a lot more) for the $q$-deformed Weyl algebra can be found in Hellström and Silvestrov [65]. In the context of quantization one meets the same operator ordering ambiguities, leading to different quantization/ordering schemes. A nice discussion can be found in Cohen [44] and de Gosson [62], where also reordering formulas are given. Operator ordering questions are also treated in "non-commutative analysis", see Maslov [102] or Nazaikinskii et al. [114]. From a more physical side, ordering results (for the first or $n$-th Weyl algebra) are, of course, given in many books or surveys; we only mention the classic book of Louisell [90] from 1964.

The structure of the paper is as follows. In Section 2, we present recent developments concerning normal ordering in the Weyl algebra $A_{1}$. In particular, combinatorial properties or interpretations for normal ordering coefficients of particular words are discussed, leading to new interpretations for generalized Stirling numbers of both kinds and the Whitney numbers. Furthermore, generalized geometric and Eulerian polynomials are considered. The connection of Spivey's identity (and a few generalizations) to normal ordering is discussed and the normal ordered expansion of the powers of a Lie derivative revisited. In Section 3, normal ordering in the $n$-th Weyl algebra is considered and a combinatorial interpretation for the normal ordering coefficients in terms of digraphs is described. Furthermore, a few other recent developments in the multi-mode case are mentioned. Section 4 presents several relatives of the quantum plane or the $q$-deformed Weyl algebra and some of the research concerning normal ordering for them. The non-commutative binomial formula is revisited in Section 5 where some general observations are made and a few particular instances are studied. In the final Section 6, diverse research related to normal ordering is briefly collected. 


\section{Normal ordering coefficients in the Weyl algebra (and slightly beyond)}

In the Introduction it was mentioned that (generalized) Stirling numbers $S_{r, s}(n, k)$ can be defined as normal ordering coefficients of $\left(V^{r} U^{s}\right)^{n}$, where $U, V$ satisfy the commutation relation of the Weyl algebra $A_{1}$, i.e.,

$$
U V-V U=I .
$$

The analogue holds true in the $q$-deformed Weyl algebra $A_{1}(q)$ where one has the commutation relation (7). Obviously, other words $\omega$ in $U, V$ can be considered and the respective normal ordering coefficients be studied. For a survey of results up to, roughly 2015, see [98]. In the following, some more recent developments are presented.

Remark 2.1 (On notation). In the following, we mostly follow the authors notations (where possible, harmonizing it a bit). Depending on the context, the generators of the Weyl algebra will sometimes be used in the abstract version (i.e., $U, V$ satisfying (11)), or in the concrete version (i.e., D, $X$ satisfying (1)), or in the physical version (i.e., $\hat{a}, \hat{a}^{\dagger}$ satisfying $\hat{a} \hat{a}^{\dagger}-\hat{a}^{\dagger} \hat{a}=I$ ). And at some places we will be a bit sloppy and follow custom to write, e.g., instead of $X^{m} D$ also $x^{m} D$ or $x^{m} \frac{d}{d x}$.

\subsection{Generalized Stirling numbers revisited}

The Stirling numbers and their generalizations have been studied by many authors, and many results were discovered several times. For the generalized Stirling numbers $S_{r, s}(n, k)$ mentioned in the Introduction, a lot of properties are known, see, e.g., $[22,63,98]$. In this section, we describe some recent work.

\subsubsection{The Mellin derivative I}

Let us start by considering powers of the Mellin derivative (or also Euler operator) $X D$, acting on functions of a real variable by $(X D f)(x)=x \frac{d f}{d x}(x)$. Using (3), one finds for the exponential polynomials (or Bell polynomials)

$$
B_{n}(x)=e^{-x}(X D)^{n} e^{x}=\sum_{k=0}^{n} S(n, k) x^{k},
$$

giving $B_{n}(1)=B_{n}$, the $n$-th Bell number. Let $\alpha, \beta$ and $r$ real or complex numbers with $(\alpha, \beta, r) \neq(0,0,0)$. The generalized Stirling numbers $S(n, k ; \alpha, \beta, r)$ of Hsu and Shiue [69] have the explicit formula

$$
S(n, k ; \alpha, \beta, r)=\frac{1}{\beta^{k} k !} \sum_{j=0}^{k}(-1)^{k-j}\left(\begin{array}{l}
k \\
j
\end{array}\right)(\beta j+r \mid \alpha)_{n},
$$

where $(t \mid \alpha)_{n}$ denotes the generalized factorial $(t \mid \alpha)_{n}=t(t-\alpha) \cdots(t-n \alpha+\alpha)$. A recent discussion of these numbers can be found in Maltenfort [91]. Introducing the associated generalized exponential polynomials by $S_{n}(x)=\sum_{k=0}^{n} S(n, k ; \alpha, \beta, r) x^{k}[69]$, Kargin and Corcino [76] showed the following analogue of (12).

Theorem 2.1. [76, Theorem 3] For $n \in \mathbb{N}_{0}$, one has

$$
S_{n}(x)=\left[\left(x^{n \alpha-r} e^{-x}\right)\right]^{1 / \beta}\left(\beta x^{1-\alpha / \beta} D\right)^{n}\left[\left(x^{r} e^{x}\right)^{1 / \beta}\right] .
$$

The operator $\left(\beta x^{1-\alpha / \beta} D\right)^{n}$ - which is a generalization of the Mellin derivative - was considered in detail in [76]. For example, the authors showed that [76, Eq. (2.5)]

$$
\left(\beta x^{1-\alpha / \beta} D\right)^{n}\left[x^{r / \beta} f(x)\right]=x^{(r-n \alpha) / \beta} \sum_{k=0}^{n} S(n, k ; \alpha, \beta, r) \beta^{k} x^{k} D^{k} f(x),
$$

which can be considered as a result in normal ordering. Note that due to the fractional exponents this is not a result in the Weyl algebra. 
Let us introduce the geometric polynomials by $\omega_{n}(x)=\sum_{k=0}^{n} S(n, k) k ! x^{k}$. The numbers $\omega_{n}(1)$ are called ordered Bell numbers (or also Fubini numbers) and are sequence A000670 in [127]. It is well-known that

$$
(X D)^{n}\left\{\frac{1}{1-x}\right\}=\omega_{n}\left(\frac{x}{1-x}\right)
$$

and that $\omega_{n}\left(\frac{x}{1-x}\right)=(1-x)^{-n} A_{n}(x)$, where $A_{n}(x)$ denotes the $n$-th Eulerian polynomial. Kargin and Corcino [76, Eq. (3.1)] introduced the generalized geometric polynomials $w_{n}(x ; \alpha, \beta, r)=\sum_{k=0}^{n} S(n, k ; \alpha, \beta, r) \beta^{k} k ! x^{k}$ and showed the following generalization of (15) in [76, Eq. (3.2)],

$$
\left(\beta x^{1-\alpha / \beta} D\right)^{n}\left\{\frac{x^{r / \beta}}{1-x}\right\}=\frac{x^{(r-n \alpha) / \beta}}{1-x} w_{n}\left(\frac{x}{1-x} ; \alpha, \beta, r\right) .
$$

Several further properties of $w_{n}(x ; \alpha, \beta, r)$ were studied in [76]. The same generalized Mellin derivative was used by Kargin and Çekim [75] to introduce higher order geometric polynomials. Generalizing (15) by letting

$$
\left(X^{r} D^{s}\right)^{n}\left\{\frac{1}{1-x}\right\}=\frac{K_{r, s, n} x^{r-1} A_{r, s, N_{r}(n)}(x)}{(1-x)^{n s+1}}
$$

Agapito [4, Definition 3.4] defined polynomials $A_{r, s, N_{r}(n)}(x)$ of degree $N_{r}(n)=r(n-1)+1$ which generalize the classical Eulerian $(r=1, s=1)$ and Narayana polynomials $(r=1, s=2)$. Normal ordering $\left(X^{r} D^{s}\right)^{n}$ was used to study several properties of these polynomials. In the same paper, the more general expansion $\left(X^{r} D^{s}\right)^{n}\left(\frac{a x+b}{c x+d}\right)$ was also considered. A combinatorial interpretation for $A_{r, s, N_{r}(n)}(x)$ in terms of a deformed excedance statistic was given recently by Agapito et al. [5]. They generalized (16) further by considering the action of an arbitrary word $\Pi D^{m-|\mathbf{r}|}=D^{r_{1}} X^{s_{1}} D^{r_{2}} X^{s_{2}} \cdots D^{r_{n}} X^{s_{n}} D^{m-|\mathbf{r}|}$ on $\left\{\frac{1}{1-x}\right\}$, where $|\mathbf{r}|=r_{1}+\cdots+r_{n}$ and $m$ is any positive integer with $m \geq \max (|\mathbf{r}|,|\mathbf{s}|)$ and showed [5, Theorem 5] that one has an expansion

$$
\Pi D^{m-|\mathbf{r}|}\left\{\frac{1}{1-x}\right\}=\frac{A_{m, \lambda^{(m)}}(x)}{(1-x)^{m+1}},
$$

where the $A_{m, \lambda(m)}$ are certain generalized Eulerian polynomials defined in [5]. By considering in $\Pi D^{m-|\mathbf{r}|}$ instead of $D$ the Jackson derivative $D_{q}$, see (8), a $q$-deformation of the above expansion was derived [5, Eq. (24)]. Xiong and Fang [135] considered closely related expansions. They studied the operators $\left(D e^{X} D\right)^{n},\left(e^{X} D^{2}\right)^{n}$ and $\left(D^{2} e^{X}\right)^{n}$ and their action on the functions $\frac{1}{1-e^{x}}$ and $\frac{e^{x}}{1-e^{x}}$. If $N(n, k)=\frac{1}{n}\left(\begin{array}{c}n \\ k+1\end{array}\right)\left(\begin{array}{c}n \\ k\end{array}\right)$ denote the Narayana numbers (A001263 in [127]), then the Narayana polynomials are given by $N_{n}(x)=\sum_{k=0}^{n-1} N(n, k) x^{k}$. Then one has, for example,

$$
\left(D e^{X} D\right)^{n}\left\{\frac{1}{1-e^{x}}\right\}=\frac{n !(n+1) ! e^{(n+1) x} N_{n}\left(e^{x}\right)}{\left(1-e^{x}\right)^{2 n+1}},
$$

see [135, Eq. (1)]. Changing the variable $Y=e^{X}$ and letting $\mathcal{D}=\frac{d}{d y}$, one obtains from (17) due to $D=Y \mathcal{D}$ the identity $\left(Y \mathcal{D} Y^{2} \mathcal{D}\right)^{n}\left\{\frac{1}{1-y}\right\}=\frac{n !(n+1) ! y^{n+1} N_{n}(y)}{(1-y)^{2 n+1}}[135$, Corollary 1]. Several similar formulas can be found in [135].

In fractional calculus, the generalized Mellin derivative $x^{\rho} D$ (with $\rho \in \mathbb{R}$ ) and its powers also plays a role, see [81] and the references given therein. Katugampola [81] considered the operators $\left(x^{r} D^{s}\right)^{n}$ for $r, s \in \mathbb{N}$, hence the generalized Stirling numbers $S_{r, s}(n, k)$. Letting $s=1$, but allowing $r \in\left\{\frac{1}{2}, \frac{1}{3}, \frac{1}{4}, \ldots\right\}$, he introduced new sequences of generalized Stirling numbers as normal ordering coefficients of powers of the generalized Mellin derivative $x^{r} D$.

Definition 2.1. [81, Definition 5.1] The generalized Stirling numbers of order $\frac{1}{2}, S^{\frac{1}{2}}(n, k)$, are defined as normal ordering coefficients of $2^{\frac{n-1}{2}}(\sqrt{x} D)^{n}$, when $n$ is odd, and of $2^{\frac{n}{2}}(\sqrt{x} D)^{n}$, when $n$ is even.

As mentioned in [81, Table 8], we have the first few expansions

$$
2(\sqrt{x} D)^{2}=D+2 x D^{2}, 2(\sqrt{x} D)^{3}=3 \sqrt{x} D^{2}+2 \sqrt{x}^{3} D^{3}, 2^{2}(\sqrt{x} D)^{4}=3 D^{2}+12 x D^{3}+4 x^{2} D^{4},
$$

giving, e.g., $S^{\frac{1}{2}}(4,1)=0, S^{\frac{1}{2}}(4,2)=3, S^{\frac{1}{2}}(4,3)=12, S^{\frac{1}{2}}(4,1)=4$, and a table with more values for $S^{\frac{1}{2}}(n, k)$ was given in [81, Table 7]. The sequence is A223168 in [127], and it was discussed in [81] that there exists a 
close connection to generalized Laguerre poynomials. Considering the corresponding Bell numbers $B_{n}^{\frac{1}{2}}$ of order $\frac{1}{2}$, the sequence starts with $1,3,5,19,39,173,407,2025, \ldots$ and can be found as A242818 in [127], where we also find the relation $B_{n}^{\frac{1}{2}}=2^{\left\lfloor\frac{n}{2}\right\rfloor} n !\left[x^{n}\right] e^{x+(x / 2)^{2}}$, where $\left[x^{n}\right] f(x)$ denotes the $n$-th coefficient in the series expansion of $f$. Thus, there is a close relation to the involution numbers (A000085 in [127]), whose exponential generating function is given by $e^{x+x^{2} / 2}$, see Mező [106]. In a similar fashion, the generalized Stirling numbers of order $\frac{1}{r}$, for $r=2,3,4, \ldots$, were defined [81, Definition 5.2] as normal ordering coefficients of $r^{\frac{n-1}{2}}\left(x^{\frac{1}{r}} D\right)^{n}$, when $n$ is odd, and of $r^{\frac{n}{2}}\left(x^{1-\frac{1}{r}} D\right)^{n}$, when $n$ is even. Tables of the first few values were given for $r=3,4,5,6$ in [81], and the corresponding sequences can be found as A223168 - A223172 in [127]. Turning to the associated Bell numbers, $B_{n}^{\frac{1}{r}}$, one obtains from the tables in [81] that the sequence of Bell numbers of order $\frac{1}{3}$ starts with $1,4,7,37,79,496, \ldots$. This sequence (and neither the ones for $r=4,5,6)$ is not contained in [127].

Problem 2.1. Give a combinatorial interpretation for the Stirling and Bell numbers of order $\frac{1}{r}$, with $r=$ $2,3,4, \ldots$ (see also Problem 4.1). In [81, Remark 7] this was posed as a problem and a relation to counting on Ferrer's boards was suggested. It was furthermore suggested to extend these considerations to arbitrary $r \in \mathbb{Q}$, see [81, Remark 8].

\subsubsection{The Mellin derivative II}

Recently, Kargin and Can [74] considered different generalizations of the Mellin derivative. Recall that the $r$-Stirling numbers of the second kind, $S^{[r]}(n, k)$, can be defined by

$$
(x+r)^{n}=\sum_{k=0}^{n} S^{[r]}(n, k) x^{\underline{k}},
$$

where $x^{\underline{k}}=x(x-1) \cdots(x-k+1)$ denotes the falling factorial, see the references given in [74] or [106]. For $r=0$, one recovers the conventional Stirling numbers. Replacing $x$ by $(x D)$, and using Boole's formula from 1844 (see [98]), $(x D)^{\underline{k}}=x D(x D-1) \cdots(x D-k+1)=x^{k} D^{k}$, one obtains the $r$-shifted version of (3),

$$
(x D+r)^{n}=\sum_{k=0}^{n} S^{[r]}(n, k) x^{k} D^{k} .
$$

Denoting by $x^{\bar{k}}=x(x+1) \cdots(x+k-1)$ the rising factorial, one has for the $r$-Lah numbers $L^{[r]}(n, k)$ a definition similar to (18), $(x+2 r)^{\bar{n}}=\sum_{k=0}^{n} L^{[r]}(n, k) x^{\underline{k}}$, see [74, Eq. (4)] and the references given there. Clearly, for $r=0$ it reduces to the conventional relation for the Lah numbers. Using the same argument as before, one obtains the following normal ordering result [74, Eq. (8)],

$$
(x D+2 r)^{\bar{n}}=\sum_{k=0}^{n} L^{[r]}(n, k) x^{k} D^{k} .
$$

For $r=0$, this gives another interpretation for the Lah numbers as normal ordering coefficients (recall from the Introduction that they also appear upon normal ordering $\left.\left(x^{2} D\right)^{n}\right)$. Applying (19) to the function $e^{x}$, one finds $(x D+2 r)^{\bar{n}} e^{x}=e^{x} L_{n}^{[r]}(x)$, where the exponential $r$-Lah polynomials are defined by $L_{n}^{[r]}(x)=\sum_{k=0}^{n} L^{[r]}(n, k) x^{k}$. Using these exponential $r$-Lah polynomials, many identities of the $r$-Lah numbers were rediscovered in [74]. There, geometric r-Lah polynomials $\mathfrak{L}_{n}^{[r]}(x)$ were introduced by $\mathfrak{L}_{n}^{[r]}(x)=\sum_{k=0}^{n} L^{[r]}(n, k) k ! x^{k}$ (compare with the definition of the geometric polynomials $\omega_{n}(x)$ in Section 2.1.1), and one has in analogy to (15)

$$
(x D+2 r)^{\bar{n}}\left\{\frac{1}{1-x}\right\}=\frac{1}{1-x} \mathfrak{L}_{n}^{[r]}\left(\frac{x}{1-x}\right),
$$

see [74, Section 7]. Using these polynomials, identities relating $r$-Lah numbers, $r$-Stirling numbers, Bernoulli numbers and (hyper-)harmonic numbers were derived in [74].

\subsubsection{Generalized Stirling numbers of the first kind}

Scherk [120] considered in 1823 also the expressions $\left(e^{x} D\right)^{n}$, and he found (in our terminology) that

$$
\left(e^{x} D\right)^{n}=e^{n x} \sum_{k=0}^{n}|s(n, k)| D^{k},
$$


i.e., the signless Stirling numbers of the first kind $(s(n, k)$ is A132393 in [127]) appear as normal ordering coefficients. This has been generalized in different directions, see [98]. Clearly, an immediate step is to consider the expansion of $\left(e^{r x} D^{s}\right)^{n}$, where $r \in \mathbb{R}$ and $s \in \mathbb{N}$. El-Desouky [53] considered a sequence $\mathbf{r}=\left(r_{1}, \ldots, r_{n}\right)$ of real numbers and a sequence $\mathbf{s}=\left(s_{1}, \ldots, s_{n}\right)$ of natural numbers and defined generalized Stirling numbers $s_{\mathbf{r}, \mathbf{s}}(k)$ as normal ordering coefficients,

$$
e^{r_{n} x} D^{s_{n}} \cdots e^{r_{2} x} D^{s_{2}} e^{r_{1} x} D^{s_{1}}=e^{|\mathbf{r}| x} \sum_{k=s_{1}}^{|\mathbf{s}|} s_{\mathbf{r}, \mathbf{s}}(k) D^{k},
$$

where $|\mathbf{r}|=r_{1}+r_{2}+\cdots+r_{n}$ (and similarly for $|\mathbf{s}|$ ), and where $s_{\mathbf{r}, \mathbf{s}}(k)=0$ for $s_{1}>k$ and $k>|\mathbf{s}|$. Choosing $\mathbf{r}=(1, \ldots, 1)$ and $\mathbf{s}=(1, \ldots, 1)$ and comparing with $(20)$, one finds that $s_{(1, \ldots, 1),(1, \ldots, 1)}(k)=|s(n, k)|$. Several properties of the numbers $s_{\mathbf{r}, \mathbf{s}}(k)$ were studied in [53]. More recently, El-Desouky et al. [54] defined certain $\beta$-numbers as follows. Let again $\mathbf{r}=\left(r_{1}, \ldots, r_{n}\right)$ be a sequence of real numbers and $\mathbf{s}=\left(s_{1}, \ldots, s_{n}\right)$ be a sequence of natural numbers. Then the $\beta$-numbers $\beta_{\mathbf{r}, \mathbf{s}}(n, k)$ are defined as normal ordering coefficients,

$$
\left(D^{s_{n}} e^{r_{n} x} D^{s_{n}}\right) \cdots\left(D^{s_{2}} e^{r_{2} x} D^{s_{2}}\right)\left(D^{s_{1}} e^{r_{1} x} D^{s_{1}}\right)=e^{|\mathbf{r}| x} \sum_{k=s_{1}}^{2|\mathbf{s}|} \beta_{\mathbf{r}, \mathbf{s}}(n, k) D^{k},
$$

where $\beta_{\mathbf{r}, \mathbf{s}}(n, 0)=1$ and $\beta_{\mathbf{r}, \mathbf{s}}(n, k)=0$ for $s_{1}>k>2|\mathbf{s}|$. In [54], the recurrence relation and explicit expression for $\beta_{\mathbf{r}, \mathbf{s}}(n, k)$ were derived and many special cases were discussed.

\subsubsection{Interpretations for generalized Stirling numbers of the second kind}

The generalized Stirling and Bell numbers have been interpreted in new combinatorial ways recently. First, we present an interpretation for the generalized Stirling and Bell numbers $S_{m, m}(n, k)$ and $B_{m, m}(n)$ according to Codara et al. [43]. Recall from the introduction that $S_{m, m}(n, k)$ is given as normal ordering coefficient of $\left(X^{m} D^{m}\right)^{n}$. We follow closely the presentation given in [43]. A $k$-colouring of a graph $G$ is a partitioning of the vertex set of $G$ into $k$ non-empty stable sets, i.e., sets not containing adjacent vertices. Each such stable set is called a colour class of the partition. Let us denote by $K_{m}$ the complete graph on $m$ vertices, and by $n K_{m}$ the disjoint union of $n$ copies of $K_{m}$, see Figure 1 for $2 K_{4}$ with a particular 5-colouring.
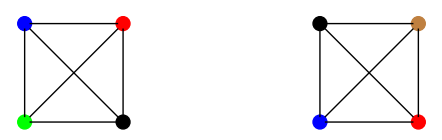

Figure 1: The graph $2 K_{4}$ with a particular 5-colouring.

Now, we can state the following result.

Theorem 2.2. [43, Proposition 2.2] The number of $k$-colourings of $n K_{m}$ is given by $S_{m, m}(n, k)$.

The proof consists in checking that the number of $k$-colourings of $n K_{m}$ satisfies the same recurrence relation as $S_{m, m}(n, k)$. As an example, $S_{2,2}(n, k)$ is A078739 in [127]. To present the interpretation of $B_{m, m}(n)$ according to [43], we consider digraphs (directed graphs) that allow loops and multiple edges in the same direction. A digraph $G$ is Eulerian if at every vertex the in-degree equals the out-degree. The edge set of an Eulerian digraph $G$ can be partitioned into directed cycles. An Eulerian digraph is called $(n, m)$-labelled if its edge set is partitioned into $n$ directed $m$-cycles, each with a distinguished first edge. See Figure 2 for an example.

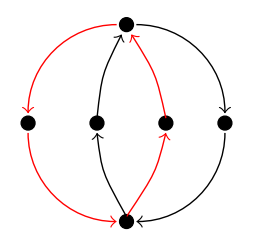

Figure 2: A $(2,4)$-labelled Eulerian digraph. 
Theorem 2.3. [43, Theorem 3.1] The number of $(n, m)$-labelled Eulerian digraphs is given by $B_{m, m}(n)$.

The proof consists in showing a bijection between the set of $(n, m)$-labelled Eulerian digraphs and the number of colourings of $n K_{m}$, and using Theorem 2.2. As an example, $B_{2,2}(n)$ is A020556 in [127].

The interpretation given in Theorem 2.2 was generalized by Engbers et al. [55] as follows. Let $\omega$ be a word in $X$ and $D$ having $m X^{\prime} s$ and $n D^{\prime} s(m \geq n)$. Then one has the unique expansion

$$
\omega=X^{m-n} \sum_{k \geq 0} S_{\omega}(k) X^{k} D^{k}
$$

where $S_{\omega}(k)$ are called Stirling numbers associated to $\omega$. A word $\omega$ in the alphabet $\{X, D\}$ is a Dyck word, if it has the same number of $X^{\prime} s$ and $D^{\prime} s$, and if, reading the word from left to right, every initial segment has at least as many $X^{\prime} s$ as $D^{\prime} s$. For a Dyck word of length at least four, we say that it is irreducible if, on deletion of a leading $X$ and a trailing $D$, the resulting word is a Dyck word, and say that it is reducible otherwise. Note that an irreducible Dyck word is of the form $X \omega^{\prime} D$, where $\omega^{\prime}$ is a Dyck word. For a Dyck word $\omega$, a graph $G_{\omega}$ is associated inductively as follows [55]:

(1) For $\omega=X D, G_{\omega}=K_{1}$ (the isolated vertex).

(2) If $\omega=X \omega^{\prime} D$ is irreducible, then $G_{\omega}=G_{\omega^{\prime}}+K_{1}$ (the graph obtained from $G_{\omega^{\prime}}$ by adding a dominating vertex).

(3) If $\omega=\omega_{1} \cdots \omega_{\ell}$ is reducible, then $G_{\omega}=G_{\omega_{1}} \cup \cdots \cup G_{\omega_{\ell}}$ (the disjoint union of the $G_{\omega_{j}}$ ).

Example 2.1. If $\omega=(X D)^{n}$, then $G_{(X D)^{n}}$ is the empty graph $G_{n}$ on $n$ vertices. Much more interesting is $\omega=\left(X^{r} D^{r}\right)^{n}$, where $G_{\left(X^{r} D^{r}\right)^{n}}=n K_{r}$, the disjoint union of $n$ copies of $K_{r}$.

Following Engbers et al. [55] (see also Duncan and Peele [49]), we introduce the following objects.

Definition 2.2. Given a graph $G$ and $k \in \mathbb{N}$, we denote by $S(G, k)$ the graphical Stirling number, that is, the number of ways of partitioning the vertex set of $G$ into $k$ nonempty classes, none of which contains both endvertices of an edge of $G$. The graphical Bell number $B_{G}$ is defined by $B_{G}=\sum_{k=0}^{|V(G)|} S(G, k)$.

Note that this definition is equivalent to the number of ways of colouring the vertices of $G$ using $k$ colours, where each colour is used for at least one vertex.

Example 2.2. If $G_{n}$ is the graph on $n$ vertices having no edges, then $S\left(G_{n}, k\right)=S(n, k)$. Combining this with Example 2.1, we find that $S(n, k)=S\left(G_{n}, k\right)=G_{(X D)^{n}}=S_{1,1}(n, k)$.

Combining Theorem 2.2 with Example 2.2, one obtains $S_{r, r}(n, k)=S\left(G_{\left(X^{r} D^{r}\right)^{n}}, k\right)$, that is, the generalized Stirling numbers $S_{r, r}(n, k)$ can be interpreted as graphical Stirling numbers of the graph $G_{\left(X^{r} D^{r}\right)^{n}}=n K_{r}$ associated to the word $\omega=\left(X^{r} D^{r}\right)^{n}$. This can be generalized to arbitrary Dyck words.

Theorem 2.4. [55, Theorem 2.3] Let $\omega$ be a Dyck word in the alphabet $\{X, D\}$. For all $k \in \mathbb{N}$ one has $S_{\omega}(k)=S\left(G_{\omega}, k\right)$.

To extend this result to arbitrary words $\omega$, we follow again [55] and let $a=a(\omega)$ be the least nonnegative integer such that all initial segments of $X^{a} \omega$ have at least as many $X^{\prime} s$ as $D^{\prime} s$, and let $b=b(\omega)$ be the unique nonnegative integer such that $X^{a} \omega D^{b}$ is a Dyck word. It is called Dyck word associated with $\omega$. Writing $\omega$ as in $(23)$, one finds

$$
X^{a} \omega D^{b}=X^{a+m-n} \sum_{k \geq 0} S_{\omega}(k) X^{k} D^{k+b}=\sum_{k \geq 0} S_{\omega}(k) X^{k+b} D^{k+b},
$$

where $a+m=b+n$ was used. Comparing this with $X^{a} \omega D^{b}=\sum_{k \geq 0} S_{X^{a} \omega D^{b}}(k) X^{k} D^{k}$, one finds $S_{\omega}(k)=$ $S_{X^{a} \omega D^{b}}(k+b)$. Since $X^{a} \omega D^{b}$ is a Dyck word, we can use Theorem 2.4 to obtain $S_{\omega}(k)=S\left(G_{X^{a} \omega D^{b}}, k+b\right)[55$, Corollary 2.5]. Let us point out that in [55] another interpretation for $S_{\omega}(k)$ in terms of graphs was given, as well as a combinatorial interpretation for the corresponding $q$-deformed generalized Stirling numbers $S_{\omega}^{q}(k)$ (appearing in the analogue of (23) but where $D$ is replaced by the Jackson derivative $D_{q}$ ). Thus, for a general 
Dyck word $\omega$ one has an interpretation for $S_{\omega}(k)$ in terms of the graphical Stirling numbers, while for the particular Dyck words $\omega=\left(X^{m} D^{m}\right)^{n}$ one has the additional interpretation in terms of $(n, m)$-labelled Eulerian digraphs. As a first step to extend the latter interpretation to arbitrary Dyck words, Codara and D'Antona [42, Theorem 3.1] constructed a bijection between the set of all Dyck words and a particular class of labelled Eulerian digraphs, the Dyck-Eulerian digraphs. For a vector $\mathbf{s}=\left(s_{1}, \ldots, s_{n}\right)$ of positive integers an Eulerian digraph is called s-labelled if its edge set is partitioned into $n$ directed cycles of length $s_{1}, \ldots, s_{n}$, each with distinguished first edge. In this terminology, the graph from Figure 2 is a $(4,4)$-labelled Eulerian digraph, and what we called above $(n, m)$-labelled Eulerian digraph is in this terminology an $(m, \ldots, m)$-labelled Eulerian digraph $(n$ times $m)$. An s-labelled Eulerian digraphs is a Dyck-Eulerian digraph if it satisfies some conditions on its cycles, see [42, Definition 2.1].

Problem 2.2. a) Given a Dyck word $\omega$ in $X$ and $D$, the generalized Stirling numbers $S_{\omega}(k)$ from (23) should have a combinatorial interpretation in terms of the Dyck-Eulerian digraph $\mathcal{E}_{\omega}$ associated to $\omega$ (according to [42, Theorem 3.1]). This problem was also mentioned by Codara and D'Antona [42]. b) Recall that the number of Dyck paths (hence, Dyck words) of length $2 n$ is given by the Catalan number $C_{n}=\frac{1}{n+1}\left(\begin{array}{c}2 n \\ n\end{array}\right)$, see A000108 in [127]. One may refine the consideration by considering Dyck paths with $\ell$ "peaks". A peak corresponds in our representation in a Dyck word to a letter $D$ which has as a left neighbour a letter X. For example, the Dyck word (and path) of Figure 3 has 3 peaks. The number of Dyck paths of length $2 n$ with exactly $\ell$ peaks is given by the Narayana number $N(n, \ell)$, see A001263 in [127]. Clearly, $C_{n}=\sum_{\ell=0}^{n} N(n, \ell)$. Can one say more in the above interpretations for $S_{\omega}(k)$ if $\omega$ has exactly $\ell$ peaks? c) In the opposite direction: As mentioned after Theorem 2.4, the interpretation for $S_{\omega}(k)$ as graphical Stirling number can be extended from Dyck words to arbitrary words. What about the interpretation in terms of some kind of Eulerian digraphs (extension of a) above)?

Galvin [59] gave another interpretation for $S_{\omega}(k)$ in terms of matchings in bipartite graphs. Associated to a Dyck word $\omega$ in $X$ and $D$ we associate a Dyck path in $\mathbb{Z}^{2}$. Recall that a Dyck path is a staircase path (a path that proceeds by taking unit steps, either in the positive $x$ direction or the positive $y$ direction) that starts in $(0,0)$, ends on the line $x=y$, and never goes below this line. By mapping $X$ (resp., $D$ ) to a step in the positive $y$ direction (resp., $x$ direction) we obtain a natural bijection between Dyck words and Dyck paths, see Figure 3 (recall that we read $\omega$ from left to right).
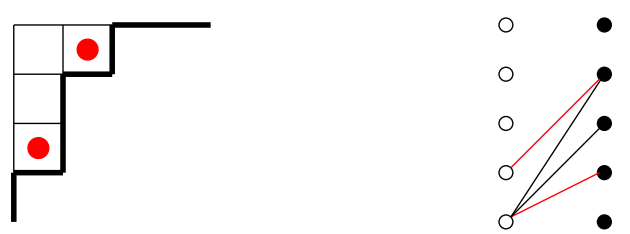

Figure 3: Dyck path and associated Ferrers board $B_{\omega}$ for $\omega=X D X X D X D D$ with a placement of two nonattacking rooks (left). Associated bipartite graph $\Gamma_{\omega}$ with matching of size $4-2=2$ in red (right).

The cells lying above the Dyck path (in the board $[0, n] \times[0, n]$, when there are $n$ letters $X$ and $D$ and we write $[0, n]=\{0,1, \ldots, n\}$ ), is the Ferrers board $B_{\omega}$ associated to $\omega$ in the convention of Varvak [131]. (Note that we obtain the same board when instead mapping $X$ (resp., $D$ ) to a step in the positive $x$ direction (resp., $y$ direction) step and let the board be formed by the cells below the path.) If we denote by $r_{k}\left(B_{\omega}\right)$ the $k$-th rook number of $B_{\omega}$, i.e., the number of ways of placing $k$ non-attacking rooks on $B_{\omega}$, then we have [59, Theorem 4] $S_{\omega}(k)=r_{n-k}\left(B_{\omega}\right)$ which is Varvak's result [131, Theorem 3.1]. Based on this interpretation, Galvin [59] associated to $B_{\omega}$ a bipartite graph $\Gamma_{\omega}$ as follows (see Figure 3 ): Introduce two partition classes $X=\left\{x_{1}, \ldots, x_{n}\right\}$ and $Y=\left\{y_{1}, \ldots, y_{n}\right\}$ and put an edge from $x_{i}$ to $y_{j}$ if and only if the cell with upper right corner $\left(x_{i}, y_{j}\right)$ belongs to $B_{\omega}$. A placement of $k$ non-attacking rooks on $B_{\omega}$ corresponds bijectively to a selection of $k$ independent edges (edges sharing no endvertices) in $\Gamma_{\omega}$, i.e., to a matching of size $k$ in $\Gamma_{\omega}$. Let us denote by $m_{k}\left(\Gamma_{\omega}\right)$ for the number of matchings of size $k$ in $\Gamma_{\omega}$. Then the above observations show the following result.

Theorem 2.5. [59, Theorem 6] For every Dyck word $\omega$ and $k \in \mathbb{N}$ one has $S_{\omega}(k)=m_{n-k}\left(\Gamma_{\omega}\right)$. 
Galvin [59, Theorem 5] gave another interpretation for $S_{\omega}(k)$ in terms of partitions of a particular indifference graph constructed from the cells below the Dyck path and those completely above it.

Before leaving this section, we would like to point out that the generalized Stirling numbers $S_{r, r}(n, k)$ - the normal ordering coefficients of $\left(X^{r} D^{r}\right)^{n}$ - appear in the context of juggling card sequences, see Butler et al. [29], thereby receiving another combinatorial interpretation. Pita-Ruiz [116] introduced generalized Stirling numbers and mentioned that they can be interpreted as normal ordering coefficients of $\left(\alpha_{0}+\alpha_{1} X D+\cdots+\alpha_{r} X^{r} D^{r}\right)^{n}$ (for suitably chosen coefficients $\alpha_{j}$ ), see [116, Eq. (73)], and announced a closer study in future research.

\subsubsection{Interpretations for generalized Stirling numbers of the first kind and Lah numbers}

In the preceding section, we considered generalized Stirling numbers $S_{\omega}(k)$ by normal ordering words in $X, D$. In that fashion, they are generalizations of the conventional Stirling numbers of the second kind, $S(n, k)$. Concerning the Stirling numbers of the first kind, one has the identity

$$
X^{n} D^{n}=\sum_{k=0}^{n} s(n, k)(X D)^{k},
$$

which one can verify by inserting (3) and using the orthogonality relation of the Stirling numbers. It is not a formula for normal ordering, but an example for bringing some word $\omega$ into a particular order. Eu et al. [56, Eq. (4)] introduced for a Dyck word $\omega$ the generalized Stirling numbers of the first kind, $s(\omega, k)$, by the expansion

$$
\omega=\sum_{k=0}^{n} s(\omega, k)(X D)^{k} .
$$

(Note that in [56] the unsigned quantities are defined as generalized Stirling numbers of the first kind.) In analogy to (24), one has

$$
X^{n} D^{n}=\sum_{k=0}^{n}(-1)^{n-k} L(n, k) X D X^{k-1},
$$

where $L(n, k)$ denote again the (unsigned) Lah numbers. Eu et al. [56, Eq. (6)] defined for any word $\omega$ with equal number of $X^{\prime} s$ and $D^{\prime} s$ and starting with an $X$, the generalized Lah numbers to the word $\omega$ by

$$
\omega=\sum_{k=0}^{n}(-1)^{n-k} L(\omega, k) X D X^{k-1} .
$$

Clearly, one can define the analogous $q$-deformed numbers $s_{q}(\omega, k)$ and $L_{q}(\omega, k)$, and this was also done in [56] (the $q$-deformed Lah numbers $L_{q}(n, k)$ resulting from the analogue of (26) seem to be a new variant not considered before). For the undeformed and deformed generalized Stirling and Lah numbers several combinatorial interpretations were given. It was shown that $|s(\omega, k)|$ and $L(\omega, k)$ can be interpreted in terms of graph decompositions into certain decreasing forests ( [56, Theorem 2.2] and [56, Theorem 2.6]). Introducing suitable weights in the forests, an interpretation for $s_{q}(\omega, k)$ and $L_{q}(\omega, k)$ can also be found ( [56, Theorem 3.1] and [56, Theorem $3.8])$.

Varvak [131, Theorem 6.1] gave an interpretation for $S_{\omega}^{q}(k)$ in terms of rook numbers on Ferrers boards, see the preceding section for the undeformed case. As analogy to this, Eu et al. [56] gave an interpretation for $s_{q}(\omega, k)$ and $L_{q}(\omega, k)$ in terms of rook numbers, too. We follow closely [56] and present their models. For a positive integer $n$, consider the square $[0, n] \times[0, n]$ in $\mathbb{Z} \times \mathbb{Z}$ having lower-left corner $(0,0)$ and upper-right corner $(n, n)$. A Dyck word with $n$ letters $X$ and $n$ letters $D_{q}$ in the $q$-deformed Weyl algebra forms a lattice path from $(0,0)$ to $(n, n)$, where $X$ (resp., $\left.D_{q}\right)$ corresponds to a step up (resp., to the right). The Ferrers board $B_{\omega}$ associated to $\omega$ is the region (within the square $[0, n] \times[0, n]$ ) below the path, see Figure 4 . (Note that the board $B_{\omega}$ is formed by the cells below the path while in the preceding section the cells above the path formed the board! In general, there exists a relation between the rook numbers of a board and the rook number of its complementary board, see Alayont and Krzywonos [6].) The rows (resp., columns) of the board are indexed from bottom to top (resp., from left to right), and the $(i, j)$ cell is the intersection of the $i$-th row with the $j$-th column. As above, a $k$-rook placement of $B_{\omega}$ is a way to place $k$ non-attacking rooks on the board $B_{\omega}$ (i.e., no two rooks are in the same row or in the same column). 

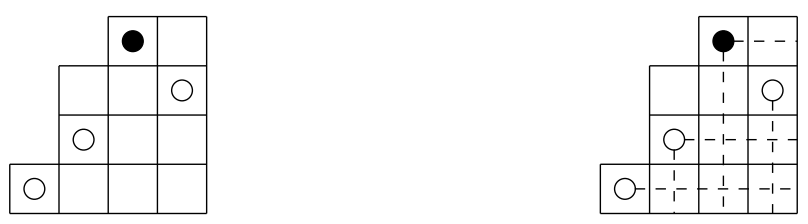

Figure 4: A 4-rook placement $\sigma \in \mathcal{R}(\omega, 3)$ for $\omega=X D_{q} X X D_{q} X D_{q} D_{q}$ with $\operatorname{inv}(\sigma)=2$.

If the Dyck word $\omega$ has $n$ letters $X$ and $n$ letters $D_{q}$, then the board $B_{\omega}$ can always accommodate $n$ nonattacking rooks. Given such an $n$-rook placement on $B_{\omega}$, a rook in cell $(i, j)$ is white if there is no rook placed in all cells $(a, b)$ with $a<i$ and $b>j$, otherwise it is black. Thus, there is no rook placed south-east of a white rook; see left of Figure 4 for an example. Let $\mathcal{R}(\omega, k)$ be the set of all $n$-rook placements on $B_{\omega}$ with $k$ white rooks. For $\sigma \in \mathcal{R}(\omega, k)$ we define the statistic $\operatorname{inv}(\sigma)$ to be the number of cells in $B_{\omega}$ that either do not have a rook above them in the same column or to the left of them in the same row, or have a black rook on them; see the right of Figure 4 for an example. Let $r_{q}(\omega, k)$ be defined as

$$
r_{q}(\omega, k)=\sum_{\sigma \in \mathcal{R}(\omega, k)} q^{-\mathrm{inv}(\sigma)} .
$$

Theorem 2.6. [56, Theorem 4.1] Let $\omega$ be a Dyck word of length $2 n$ in the alphabet $\left\{X, D_{q}\right\}$ where $X, D_{q}$ satisfy the commutation relation $D_{q} X-q X D_{q}=I$ of the $q$-deformed Weyl algebra. Then $\left|s_{q}(\omega, k)\right|=r_{q}(\omega, k)$, i.e.,

$$
\omega=\sum_{k=0}^{n}(-1)^{n-k} r_{q}(\omega, k)\left(X D_{q}\right)^{k} .
$$

Note that setting $q=1$, one finds $|s(\omega, k)|=|\mathcal{R}(\omega, k)|$. Let us turn to the generalized Lah numbers. For any word $\omega$ with $n$ letters $X$ and $n$ letters $D_{q}$ which starts with $X$, the bottom row of $B_{\omega}$ has $n$ cells. Let $B_{\omega}^{*}$ be the board obtained from $B_{\omega}$ by deleting the bottom row. Let $\mathcal{U}(\omega, k)$ be the collection of $k$-rook placements on $B_{\omega}^{*}$, see Figure 5. For $\sigma \in \mathcal{U}(\omega, k)$ we define the statistic inv' $(\sigma)$ to be the number of cells in $B_{\omega}^{*}$ that either have a rook on them, or do not have a rook above them in the same column or to the left of them in the same row, see Figure 5.
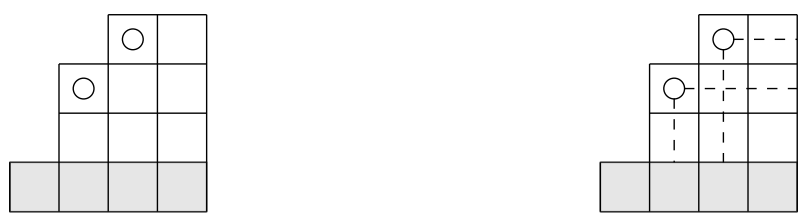

Figure 5: A rook placement $\sigma \in \mathcal{U}(\omega, 2)$ for $\omega=X D_{q} X X D_{q} X D_{q} D_{q}$ with $\operatorname{inv}^{\prime}(\sigma)=3$.

Let $u_{q}(\omega, k)$ be defined as

$$
u_{q}(\omega, k)=\sum_{\sigma \in \mathcal{U}(\omega, k)} q^{-\mathrm{inv}^{\prime}(\sigma)}
$$

Theorem 2.7. [56, Theorem 4.4] Let $\omega$ be a Dyck word of length $2 n$ in the alphabet $\left\{X, D_{q}\right\}$ starting with an $X$ where $X, D_{q}$ satisfy the commutation relation $D_{q} X-q X D_{q}=I$ of the $q$-deformed Weyl algebra. Then $L_{q}(\omega, k)=u_{q}(\omega, n-k)$, i.e.,

$$
\omega=\sum_{k=0}^{n}(-1)^{n-k} u_{q}(\omega, n-k) X D_{q}^{k} X^{k-1}
$$

(Note that there seems to be a typo in the statement of Theorem 4.4 in [56] where $L_{q}(\omega, k)=u_{q}(\omega, k)$ is stated while the proof gives $L_{q}(\omega, k)=u_{q}(\omega, n-k)$.) Setting $q=1$, one obtains $L(\omega, k)=|\mathcal{U}(\omega, n-k)|$. 


\subsection{The $(q, r)$-Whitney numbers as normal ordering coefficients}

Recall that Mező [105] introduced the $r$-Whitney numbers $w_{m, r}(n, k)$ and $W_{m, r}(n, k)$ of the first and second kind as the coefficients in the expressions

$$
m^{n} x^{\underline{n}}=\sum_{k=0}^{n} w_{m, r}(n, k)(m x+r)^{k}, \quad(m x+r)^{n}=\sum_{k=0}^{n} m^{k} W_{m, r}(n, k) x^{\underline{k}},
$$

where $x^{\underline{m}}=x(x-1) \cdots(x-m+1)$ denotes the falling factorial. See Mező [106] for more literature on these numbers and the different disguises in which they appeared before [105]. They constitute a particular subfamily of the generalized Stirling numbers $S(n, k ; \alpha, \beta, r)$ of Hsu and Shiue [65] given in $(13), w_{m, r}(n, k)=$ $S(n, k ; m, 0,-r)$ and $W_{m, r}(n, k)=S(n, k ; 0, m, r)$, as was discussed recently by Maltenfort [91]. For $m=1$ and $r=0$, they reduce to the conventional Stirling numbers of the first and second kind, i.e., $w_{1,0}(n, k)=s(n, k)$ and $W_{1,0}(n, k)=S(n, k)$. For other choices of $m$ and $r$ one recovers the $r$-Stirling numbers and Whitney numbers, see the discussion in Mangontarum and Katriel [95].

Mangontarum and Katriel [95] defined $(q, r)$-Whitney numbers - as a $q$-deformed generalization of the $r$ Whitney numbers - explicitly as normal ordering coefficients. They first showed that one can rewrite (27) in terms of the bosonic annihilation and creation operators $\hat{a}$ and $\hat{a}^{\dagger}$ mentioned above (and satisfying the commutation relation $\hat{a} \hat{a}^{\dagger}-\hat{a}^{\dagger} \hat{a}=I$ ) as

$$
m^{n}\left(\hat{a}^{\dagger}\right)^{n} \hat{a}^{n}=\sum_{k=0}^{n} w_{m, r}(n, k)\left(m \hat{a}^{\dagger} \hat{a}+r\right)^{k}, \quad\left(m \hat{a}^{\dagger} \hat{a}+r\right)^{n}=\sum_{k=0}^{n} m^{k} W_{m, r}(n, k)\left(\hat{a}^{\dagger}\right)^{k} \hat{a}^{k} .
$$

Note that, for $m=1$ and $r=0$, one finds for the right-hand side $\left(\hat{a}^{\dagger} \hat{a}\right)^{n}=\sum_{k=0}^{n} S(n, k)\left(\hat{a}^{\dagger}\right)^{k} \hat{a}^{k}$, the basic result of Katriel [77] mentioned in the Introduction (where we used $W_{1,0}(n, k)=S(n, k)$ ). To turn to the $q$-deformed generalization, the idea of [95] is to substitute in (28) the operators $\hat{a}$ and $\hat{a}^{\dagger}$ by their $q$-deformed version $\hat{a}_{q}, \hat{a}_{q}^{\dagger}$ (encountered in Example 1.3) satisfying the commutation relation (7) of the $q$-deformed Weyl algebra, i.e., $\hat{a}_{q} \hat{a}_{q}^{\dagger}-q \hat{a}_{q}^{\dagger} \hat{a}_{q}=I$. Thus, the $(q, r)$-Whitney numbers of the first and second $k i n d, w_{m, r, q}(n, k)$ and $W_{m, r, q}(n, k)$, were defined (see [95, Definition 2]) as coefficients in the expressions

$$
m^{n}\left(\hat{a}_{q}^{\dagger}\right)^{n} \hat{a}_{q}^{n}=\sum_{k=0}^{n} w_{m, r, q}(n, k)\left(m \hat{a}_{q}^{\dagger} \hat{a}_{q}+r\right)^{k}, \quad\left(m \hat{a}_{q}^{\dagger} \hat{a}_{q}+r\right)^{n}=\sum_{k=0}^{n} m^{k} W_{m, r, q}(n, k)\left(\hat{a}_{q}^{\dagger}\right)^{k} \hat{a}_{q}^{k}
$$

with appropriate initial conditions. Thus, the numbers $W_{m, r, q}(n, k)$ were defined explicitly as normal ordering coefficients. Using the commutation relation and the well-known relations

$$
\left(\hat{a}_{q}^{\dagger}\right)^{n} \hat{a}_{q}^{n}=\sum_{k=0}^{n}(-1)^{n-k} s_{q}(n, k)\left(\hat{a}_{q}^{\dagger} \hat{a}\right)_{q}^{k}, \quad\left(\hat{a}_{q}^{\dagger} \hat{a}_{q}\right)^{n}=\sum_{k=0}^{n} S_{q}(n, k)\left(\hat{a}_{q}^{\dagger}\right)^{k} \hat{a}_{q}^{k},
$$

where $s_{q}(n, k)$ and $S_{q}(n, k)$ denote the $q$-deformed Stirling numbers of the first and second kind, one can show the following results by induction.

Theorem 2.8. [95, Theorem 3] The (q,r)-Whitney numbers satisfy the following identities:

$$
w_{m, r, q}(n, k)=(-1)^{n-k} \sum_{i=k}^{n}\left(\begin{array}{l}
i \\
k
\end{array}\right) r^{i-k} m^{n-i} s_{q}(n, i), \quad W_{m, r, q}(n, k)=\sum_{i=k}^{n}\left(\begin{array}{c}
n \\
i
\end{array}\right) r^{n-i} m^{i-k} S_{q}(i, k) .
$$

Theorem 2.9. [95, Theorem 6] The (q,r)-Whitney numbers satisfy the following recurrence relations:

$$
\begin{aligned}
w_{m, r, q}(n+1, k) & =q^{-n}\left(w_{m, r, q}(n, k-1)-\left(m[n]_{q}+r\right) w_{m, r, q}(n, k)\right), \\
W_{m, r, q}(n+1, k) & =q^{k-1} W_{m, r, q}(n, k-1)+\left(m[k]_{q}+r\right) W_{m, r, q}(n, k) .
\end{aligned}
$$

Many properties of these numbers can be found in [95] (e.g., orthogonality relations, other recurrence relations, the specialization to other well-known numbers). One final point to mention is that an analogue of the Bell polynomials $B(x)=\sum_{k=0}^{n} S(n, k) x^{k}$ (note that $B(1)=B_{n}$, the $n$-th Bell number) was considered in [95]. The $(q, r)$-Dowling polynomials were defined [95, Definition 14] by $D_{m, r, q}(n, x)=\sum_{k=0}^{n} W_{m, r, q}(n, k) x^{k}$ (with $D_{m, r, q}(n, 1)$ the $(q, r)$-Dowling numbers) and many properties were shown, in particular the connection to coherent states and a Dobiński-like formula. The $(q, r)$-Whitney numbers were further studied by Bent-Usman et al. [18] and Mangontarum [92]. A different $q$-deformation of the $r$-Whitney numbers of the second kind was introduced by Corcino et al. [47]. 


\subsection{The Spivey identity revisited}

In 2008, Spivey [128, Eq. (3)] found the following new identity for the conventional Bell numbers,

$$
B_{n+m}=\sum_{k=0}^{n} \sum_{j=0}^{m} j^{n-k}\left(\begin{array}{l}
n \\
k
\end{array}\right) S(m, j) B_{k}
$$

Since then, this identity has been generalized in several directions (e.g., extension to different generalized Bell numbers - including $q$-deformed variants, dual identities), see, e.g., [98, 106]. From this huge amount of papers we would like to point out some which have a relation to normal ordering. Immediately after [128] had appeared, Katriel [79] derived a $q$-deformed variant of (30) by considering the operators $X$ and $D_{q}$ (the Jackson derivative) satisfying the commutation relation $D_{q} X-q X D_{q}=I$ of the $q$-deformed Weyl algebra. In fact,

$$
\left(X D_{q}\right)^{n+m}=\left(X D_{q}\right)^{n} \sum_{j=0}^{m} S_{q}(n, j) X^{j} D_{q}^{j}=\sum_{j=0}^{m} S_{q}(m, j) X^{j}\left([j]_{q}+q^{j}\left(X D_{q}\right)\right)^{n} D_{q}^{j}
$$

where in the first equality the $q$-analogue of (3) was used and in the second $\left(X D_{q}\right) X^{n}=X^{n}\left([n]_{q}+q^{n}\left(X D_{q}\right)\right)$. Expanding the binomial, one has

$$
\left(X D_{q}\right)^{n+m}=\sum_{j=0}^{m} \sum_{k=0}^{n} S_{q}(m, j)[j]_{q}^{n-k}\left(\begin{array}{l}
n \\
k
\end{array}\right) q^{j k} X^{j}\left(X D_{q}\right)^{k} D_{q}^{j} .
$$

Applying this to $e_{q}(x)=\sum_{l=0}^{\infty} \frac{x^{l}}{[l]_{q} !}$ and using $\frac{1}{e_{q}(x)}\left(X D_{q}\right)^{n} e_{q}(x)=B_{n}(q ; x)$, one obtains upon setting $x=1$

$$
B_{n+m}(q)=\sum_{k=0}^{n} \sum_{j=0}^{m}[j]_{q}^{n-k}\left(\begin{array}{l}
n \\
k
\end{array}\right) S_{q}(m, j) q^{j k} B_{k}(q)
$$

the $q$-deformed Spivey identity due to Katriel [79]. Clearly, letting $q=1$, one recovers (30). In the undeformed case, the same proof can be used for $X$ and $D$ satisfying (1). This line of reasoning was pursued further by Mangontarum and Dibagulun [94] who derived as a further generalization of (31) an analogous identity for the $r$-Dowling polynomials (hence, $r$-Dowling numbers). Crucial for their approach using normal ordering was the identity (see [94, Theorem 1])

$$
(m X D+r)^{n}=\sum_{k=0}^{n} m^{k} W_{m, r}(n, k) X^{k} D^{k}
$$

which is equivalent to the second equation of (28). Mangontarum [93] derived shortly later an identity for the $(q, r)$-Dowling polynomials $D_{m, r, q}(n, x)$, see Section 2.2, from which the results of [94] follow by letting $q=1$. Thus, we will directly mention this result. Denoting the falling $q$-factorial by $[x]_{q, j}=[x]_{q}[x-1]_{q} \cdots[x-k+1]_{q}$, we can state the following result [93, Eq. (24)],

$$
D_{m, r, q}(n+\ell, x)=\sum_{k=0}^{n} \sum_{j=0}^{\ell} m^{j}\left(\begin{array}{l}
n \\
k
\end{array}\right) W_{m, r, q}(\ell, j)\left(m[j]_{q}+r\right)^{n-k} q^{j k} D_{m, 0, q}(k, x)[x]_{q, j} .
$$

Setting $x=1, m=1$ and $r=0$, one recovers due to $W_{1,0, q}(\ell, j)=S_{q}(\ell, j)$ identity (31). The proof of (33) follows roughly the argument presented above for (31). For (33), the crucial ingredient is the second equation of (29), and the result follows by comparing the result of two different ways of expanding $\left(m \hat{a}_{q}^{\dagger} \hat{a}_{q}+r\right)^{n+\ell}$ and using ordering results in the $q$-deformed Weyl algebra.

Very recently, Oussi [115] obtained a nice $(p, q)$-deformed version of the Spivey identity. To achieve this, it was - apart from using the $(p, q)$-derivative $D_{p, q}$ - necessary to consider in addition the Fibonacci operator $N_{p}$ and its nontrivial commutation relation with $X$ and $D_{p, q}$. (Cigler [40] had used this operator - with symbol $\varepsilon-$ in his study of $X, D_{q}$ satisfying $D_{q} X-q X D_{q}=I$ where one has $D_{q} X-X D_{q}=N_{q}$.). Thus, let us follow [115] and introduce for two deformation parameters $p, q$ the following operators by their action on functions,

$$
\left(D_{p, q} f\right)(x)=\frac{f(p x)-f(q x)}{x(p-q)}, \quad\left(N_{p} f\right)(x)=f(p x) .
$$


Clearly, for $p=1$, one recovers the Jackson derivative, $D_{1, q}=D_{q}$, see (8), and $N_{1}=I$ is the identity. These operators satisfy the commutation relations [115, Eq. (7) - (9)]

$$
D_{p, q} X-q X D_{p, q}=N_{p}, \quad N_{p} X=p X N_{p}, \quad D_{p, q} N_{p}=p N_{p} D_{p, q} .
$$

Denoting the $(p, q)$-deformed numbers by $[n]_{p, q}=\frac{p^{n}-q^{n}}{p-q}$, one shows $D_{p, q} X^{n}=q^{n} X^{n} D_{p, q}+[n]_{p, q} X^{n-1} N_{p}$ by induction [115, Proposition 1]. This is the main ingredient for the following $(p, q)$-generalization of (3).

Theorem 2.10. [115, Proposition 3] Let $n \in \mathbb{N}$. Then one has

$$
\left(X D_{p, q}\right)^{n}=\sum_{k=0}^{n} S^{p, q}(n, k) X^{k} N_{p}^{n-k} D_{p, q}^{k}
$$

where $S^{p, q}(n, k)$ denotes the $(p, q)$-deformed Stirling numbers of the second kind with $S^{p, q}(0,0)=1$ and satisfying the recurrence relation $S^{p, q}(n, k)=p^{n-k} q^{k-1} S^{p, q}(n-1, k-1)+[k]_{p, q} S^{p, q}(n-1, k)$.

In [98, Section 2.4], one can find a discussion of closely related $(p, q)$-deformed Stirling numbers and also many references. The $(p, q)$-deformed Bell numbers are defined by $B_{n}(p, q)=\sum_{k=0}^{n} S^{p, q}(n, k)$. To derive a $(p, q)$-analogue of (31) in a similar operational fashion, one introduces the $(p, q)$-exponential function $e_{p, q}(x)$ by

$$
e_{p, q}(x)=\sum_{n=0}^{\infty} p^{\left(\begin{array}{c}
n \\
2
\end{array}\right)} \frac{x^{n}}{[n]_{p, q} !}
$$

where $[n]_{p, q} !=[n]_{p, q}[n-1]_{p, q} \cdots[1]_{p, q} !$ with $[0]_{p, q} !=1$, and uses that $D_{p, q}^{n} e_{p, q}(x)=p^{\left(\begin{array}{c}n \\ 2\end{array}\right)} e_{p, q}\left(p^{n} x\right)$. Applying both sides of (35) to $e_{p, q}(x)$ and dividing by $e_{p, q}\left(p^{n} x\right)$, we obtain the identity (a $(p, q)$-generalization of the one mentioned immediately before (31))

$$
\frac{1}{e_{p, q}\left(p^{n} x\right)}\left(X D_{p, q}\right)^{n} e_{p, q}(x)=\sum_{k=0}^{n} \tilde{S}^{p, q}(n, k) x^{k}=\tilde{B}_{n}(p, q ; x)
$$

where $\tilde{S}^{p, q}(n, k)=p^{\left(\begin{array}{c}n \\ 2\end{array}\right)} S^{p, q}(n, k)$ is a new variant of the $(p, q)$-deformed Stirling numbers, with $\tilde{B}_{n}(p, q ; x)$ being the associated $(p, q)$-deformed Bell polynomials. Adapting Katriel's proof given above (i.e., by expanding here $\left(X D_{p, q}\right)^{n+m}$ in two different ways), Oussi [115, Theorem 6] derived the following $(p, q)$-deformation of Spivey's identity,

$$
\tilde{B}_{n+m}(p, q ; 1)=\sum_{k=0}^{m} \sum_{j=0}^{n}\left(\begin{array}{c}
n \\
j
\end{array}\right)[k]_{p, q}^{n-j} \tilde{S}^{p, q}(m, k) q^{j k} \tilde{B}_{j}\left(p, q ; p^{n+m-j}\right) .
$$

Problem 2.3. The $(q, r)$-deformed Whitney numbers of the second kind $W_{m, r, q}(n, k)$ were defined by (29), which can equivalently be written as $\left(m X D_{q}+r\right)^{n}=\sum_{k=0}^{n} m^{k} W_{m, r, q}(n, k) X^{k} D_{q}^{k}$ (and, similarly, for those of the first kind $\left.w_{m, r, q}(n, k)\right)$. For both kinds of $r$-Whitney numbers a $(p, q)$-deformed version has been introduced [96, 118]. In view of (35) and the just mentioned definition of $W_{m, r, q}(n, k)$, it is tempting to introduce by

$$
\left(m X D_{p, q}+r\right)^{n}=\sum_{k=0}^{n} m^{k} W_{m, r, p, q}(n, k) X^{k} N_{p}^{n-k} D_{p, q}^{k}
$$

in an operational fashion $(p, q, r)$-deformed Whitney numbers of the second kind $W_{m, r, p, q}(n, k)$. What is their relation to those discussed in the literature mentioned? Similarly for those of the first kind $w_{m, r, p, q}(n, k)$ ? What about the associated $(p, q, r)$-Dowling polynomials and $(p, q, r)$-Dowling numbers?

\subsection{The coefficients of powers of an arbitrary Lie derivative}

Let $c(x)$ be a sufficiently smooth function (e.g., a polynomial) and consider the operator $c(x) \frac{d}{d x}=c(x) D$. Let us use the convention $c_{k}=D^{k} c$. Then we can introduce the normal ordering coefficients $A_{n, k}=A_{n, k}\left(c, c_{1}, \ldots, c_{n-k}\right)$ by

$$
(c(x) D)^{n}=\sum_{k=1}^{n} A_{n, k} D^{k} .
$$


The first few expansions are given by

$$
(c D)^{2}=\left(c c_{1}\right) D+\left(c^{2}\right) D^{2}, \quad(c D)^{3}=\left(c c_{1}^{2}+c^{2} c_{2}\right) D+\left(3 c^{2} c_{1}\right) D^{2}+\left(c^{3}\right) D^{3} .
$$

By induction, one can show that $A_{n+1,1}=c D A_{n, 1}, A_{n, n}=c^{n}$ and for $2 \leq k \leq n$

$$
A_{n+1, k}=c A_{n, k-1}+c D A_{n, k}
$$

The numbers appearing in $A_{n, k}$ can be found as sequence A139605 in [127]. The struggle to describe the coefficients $A_{n, k}$ explicitly or in a handsome combinatorial fashion began with Scherk in 1823 [120], see in particular the discussion in [22, Appendix A]. For $c(x)=x^{p}, p \geq 1$, and $c(x)=e^{x}$ Scherk found a solution for the coefficients $A_{n, k}$ (namely, what we nowadays identify as generalized Stirling numbers $S_{p, 1}(n, k)$ and the unsigned Stirling numbers of the first kind $|s(n, k)|$, see the Introduction and (20)). Two modern classic papers are Comtet [45] from 1973 who gave an explicit formula for the $A_{n, k}$ for arbitrary $c(x)$, and Bergeron and Reutenauer [19] from 1987 who described the coefficients $A_{n, k}$ in terms of certain increasing trees. This connection to increasing trees was rediscovered several times, in particular for $c(x)=x^{p}$ (see [22] for a thorough discussion and also [98] for references). More recently, new interpretations were given for the coefficients $A_{n, k}$ in the case of arbitrary $c(x)$.

Let us start with the description given by Benkart et al. [16]. Recall that a partition $\lambda=\left(\lambda_{1}, \lambda_{2}, \ldots, \lambda_{\ell}\right)$ is a weakly decreasing sequence of nonnegative integers. Each $\lambda_{k}$ is called a part of $\lambda$. The sum of the parts of a partition $\lambda$ is denoted by $|\lambda|$. If $|\lambda|=n$, then we say that $\lambda$ is a partition of $n$, denoted by $\lambda \vdash n$. The length of $\lambda$, denoted by $\ell(\lambda)$ is the maximum index $j$ such that $\lambda_{j}>0$. We also let $c_{\lambda}=c_{\lambda_{1}} c_{\lambda_{2}} \cdots c_{\lambda_{\ell}}$. The structure of the $A_{n, k}$ can then be described as follows.

Theorem 2.11. [16, Lemma 8.1] Let $A_{n, k}$ be defined by (36). There exist positive integers a $(n, \lambda)$ such that

$$
A_{n, k}=\sum_{\lambda \vdash n-k} a(n, \lambda) c^{n-\ell(\lambda)} c_{\lambda} .
$$

For example, for $A_{3,1}$ there are two partitions $\lambda=(2)$ (with $\ell(\lambda)=1$ ) and $\nu=(1,1)($ with $\ell(\nu)=2)$ of 2 , giving $A_{3,1}=a(3, \lambda) c^{2} c_{2}+a(3, \nu) c^{1} c_{1} c_{1}$, in accordance with (37). From (39), one can also read off that $A_{n, n}=a(n, \emptyset) c^{n}$ (and, in fact, $a(n, \emptyset)=1$ as mentioned above). Note that closely related expressions were given by Mohammad-Noori [108]. Recalling that in the case $c(x)=x^{p}$ the normal ordering coefficients are the generalized Stirling numbers $S_{p, 1}(n, k)$, one finds by comparison (see Briand et al. [26, Eq. (4.6)])

$$
S_{p, 1}(n, k)=\sum_{\lambda \vdash n-k} a(n, \lambda) \prod_{i=1}^{\ell(\lambda)} p \frac{\lambda_{i}}{}
$$

where the falling factorial $p \frac{\lambda_{i}}{=} p(p-1) \cdots\left(p-\lambda_{i}+1\right)$ is used. The above discussion was recently generalized by Briand et al. [26] to the powers of $\left(c(x) D^{d}\right)$, with $d \in \mathbb{N}$. Writing

$$
\left(c(x) D^{d}\right)^{n}=\sum_{k=1}^{n d} B_{n, k} D^{k},
$$

the structure of the coefficients $B_{n, k}$ can then be described as follows.

Theorem 2.12. [26, Proposition 6.8] Let $B_{n, k}$ be defined by (41). There exist positive integers b( $\left.n, \lambda\right)$, where $\lambda$ runs through the set of partitions of size $0 \leq|\lambda| \leq(n-1) d$ with at most $n-1$ parts, such that

$$
B_{n, k}=\sum_{\substack{\lambda \vdash n d-k \\ \ell(\lambda) \leq n-1}} b(n, \lambda) c^{n-\ell(\lambda)} c_{\lambda} .
$$

In [26], one can find also an explicit formula for the coefficients $b(n, \lambda)$ which generalizes Comtet's formula (for the case $d=1$ ). Similar to $d=1$ considered above, the coefficients in the case $c(x)=x^{p}$ are the generalized Stirling numbers $S_{p, d}(n, k)$. For $p \geq d$, one has the following analogue of (40) [26, Proposition 6.18],

$$
S_{p, d}(n, k)=\sum_{\substack{\lambda \vdash n d-k \\ \ell(\lambda) \leq n-1}} b(n, \lambda) \prod_{i=1}^{\ell(\lambda)} p^{\lambda_{i}} .
$$


Let us turn to another recent interpretation for the normal ordering coefficients given recently by Han and Ma [64]. An integer sequence $\mathbf{e}=\left(e_{1}, e_{2}, \ldots, e_{n}\right)$ is called an inversion sequence of length $n$, if $0 \leq e_{i}<i$ for all $1 \leq i \leq n$. Let $I_{n}$ be the set of all inversion sequences of length $n$. For $\mathbf{e} \in I_{n}$, let $|\mathbf{e}|_{j}=\#\left\{i \mid e_{i}=j, 1 \leq i \leq n\right\}$, for $0 \leq j \leq n-1$. Define on $I_{n}$ the map

$$
\psi(\mathbf{e})=c \cdot c_{|\mathbf{e}|_{1}} c_{|\mathbf{e}|_{2}} \cdots c_{|\mathbf{e}|_{n-1}} D^{|\mathbf{e}|_{0}},
$$

(note that in [64] a map $\phi(\mathbf{e})$ is defined which includes the action on $f$, i.e., $\phi(\mathbf{e})=\psi(\mathbf{e}) f)$.

Theorem 2.13. [64, Theorem 5] For $n \in \mathbb{N}$, one has

$$
(c D)^{n}=\sum_{\mathbf{e} \in I_{n}} \psi(\mathbf{e})=\sum_{\mathbf{e} \in I_{n}} c \cdot c_{|\mathbf{e}|_{1}} c_{|\mathbf{e}|_{2}} \cdots c_{|\mathbf{e}|_{n-1}} D^{|\mathbf{e}|_{0}} .
$$

As an example, let us consider $n=3$ (see [64, Example 6]). Here we have the set of inversion sequences $I_{3}=\{(0,0,0),(0,0,1),(0,0,2),(0,1,0),(0,1,1),(0,1,2)\}$. The set of corresponding $\psi(\mathbf{e})$ is given by $\left\{c c c D^{3}, c c_{1} c D^{2}, c c c_{1} D^{2}, c c_{1} c D^{2}, c c_{2} c D, c c_{1} c_{1} D\right\}$. Summing these terms gives $\left(c c_{1}^{2}+c^{2} c_{2}\right) D+\left(3 c^{2} c_{1}\right) D^{2}+\left(c^{3}\right) D^{3}$, in accordance with (37).

Note that already in the example with $n=3$ one groups those terms which produce the same product. In general, let us follow [64] and say that the type of $n$ is a pair $(k, \mu)$, denoted by $(k, \mu) \vdash n$, where $k \in\{0,1, \ldots, n\}$ and $\mu=\left(\mu_{1}, \ldots, \mu_{n-1}\right)$ is a partition of $n-k$. Let $\left(|\mathbf{e}|_{0}, \mu(\mathbf{e})\right)$ be the type of $\mathbf{e} \in I_{n}$, where $\mu(\mathbf{e})$ is the decreasing order of $|\mathbf{e}|_{1}, \ldots,|\mathbf{e}|_{n-1}$. For each type $(k, \mu)$ of $n$, let $p_{k, \mu}$ be the number of inversion sequences of type $(k, \mu)$. We can then write (44) equivalently as a sum over all types $(k, \mu)$ of $n[64$, Eq. (12)],

$$
(c D)^{n}=\sum_{(k, \mu) \vdash n} p_{k, \mu} c c_{\mu} D^{k}=\sum_{(k, \mu) \vdash n} p_{k, \mu} c c_{\mu_{1}} c_{\mu_{2}} \cdots c_{\mu_{n-1}} D^{k} .
$$

Han and Ma [64] introduced for each type $(k, \mu)$ of $n$ a $k$-Young tableau of shape $(k, \mu)$ and introduced a $g$-index $G_{Z}$ for such Young tableaus $Z$. The $p_{k, \mu}$ can then be expressed as $p_{k, \mu}=\sum_{Z} G_{Z}$, where the sum is over all $k$-Young tableau of shape $(k, \mu)$, see [64, Theorem 12] and the discussion there for more details. Applications to Eulerian (and other) polynomials were given in [64].

In another direction, Schreiber [124] developed a unifying framework for dealing with Bell polynomials and related extensions and discussed the expansion $(c D)^{n}$ from the perspective of this framework [124, Section 5.6].

Problem 2.4. Recall the rather straightforward generalization from Theorem 2.11 (for $\left.(c D)^{n}\right)$ to Theorem 2.12 $\left(\right.$ for $\left.\left(c D^{d}\right)^{n}\right)$ when describing the normal ordering coefficients in terms of partitions. What is the analogue of Theorem 2.13 when describing $\left(c D^{d}\right)^{n}$, i.e., what kind of generalization of inversion sequences is needed? When collecting the terms in an analogous fashion to (45), what is the appropriate interpretation for the coefficients $\left(p_{k, \mu}^{(d)}\right.$, say)? What can one say in both cases when one considers the q-deformation, i.e., the Jackson derivative $D_{q}$ instead of $D$ ?

\subsection{A connection to combinatorial Hopf algebras}

In this section, we point out an interesting connection to combinatorial Hopf algebras. We will be brief since to go into this in detail would take us too far afield. Blasiak et al. [21] introduced in 2010 a combinatorial algebra $\mathcal{G}$ of acyclic directed graphs with a surjective morphism to the Weyl algebra $A_{1}$ (generated by $\hat{a}$ and $\hat{a}^{\dagger}$ ). Very roughly, the combinatorial objects in $\mathcal{G}$ are acyclic directed graphs with incoming and outgoing edges (denoted by $\Gamma^{-}$and $\Gamma^{+}$). The partial product of two such graphs $\Gamma_{1}$ and $\Gamma_{2}$ is the composition given by matching some or all of the outgoing edges of $\Gamma_{1}$ with some or all of the incoming edges of $\Gamma_{2}$. Note that if one matches exactly $i$ lines, there are $\left(\begin{array}{c}\left|\Gamma_{2}^{-}\right| \\ i\end{array}\right)\left(\begin{array}{c}\left|\Gamma_{1}^{+}\right| \\ i\end{array}\right) i$ ! possibilities. The product $\Gamma_{1} * \Gamma_{2}$ is the sum over all possible partial products. There is a corresponding coproduct and Hopf algebra structure on $\mathcal{G}$. The map $\psi$ from $\mathcal{G}$ to $A_{1}$ is as follows:

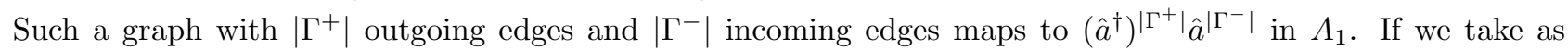
basis the normal ordered products $\left\{\left(\hat{a}^{\dagger}\right)^{k} \hat{a}^{l}\right\}_{k, l \in \mathbb{N}_{0}}$, the product of two basis elements $\left(\hat{a}^{\dagger}\right)^{p} \hat{a}^{q}$ and $\left(\hat{a}^{\dagger}\right)^{k} \hat{a}^{l}$ is determined by normal ordering $\hat{a}^{q}\left(\hat{a}^{\dagger}\right)^{k}$, i.e.,

$$
\left(\hat{a}^{\dagger}\right)^{p} \hat{a}^{q}\left(\hat{a}^{\dagger}\right)^{k} \hat{a}^{l}=\sum_{i=0}^{\min (q, k)}\left(\begin{array}{l}
q \\
i
\end{array}\right)\left(\begin{array}{l}
k \\
i
\end{array}\right) i !\left(\hat{a}^{\dagger}\right)^{p+k-i} \hat{a}^{q+l-i} .
$$


Note that the basic input for (46) is the normal ordering formula

$$
\hat{a}^{q}\left(\hat{a}^{\dagger}\right)^{k}=\sum_{i=0}^{\min (q, k)} r_{i}\left(B_{q, k}\right)\left(\hat{a}^{\dagger}\right)^{k-i} \hat{a}^{q-i}
$$

where $r_{i}\left(B_{q, k}\right)=\left(\begin{array}{c}q \\ i\end{array}\right)\left(\begin{array}{c}k \\ i\end{array}\right) i$ ! is the $i$-th rook number of the rectangular board $B_{q, k}$ with $q$ rows and $k$ columns (or vice versa), see Sections 2.1.4 and 2.1.5.

Thus, if one has in $\mathcal{G}$ the graph $\Gamma_{1}$ (resp., $\Gamma_{2}$ ) with $\left|\Gamma_{1}^{-}\right|$(resp., $\left|\Gamma_{2}^{-}\right|$) incoming and $\left|\Gamma_{1}^{+}\right|$(resp., $\left.\left|\Gamma_{2}^{+}\right|\right)$outgoing edges, then $\psi\left(\Gamma_{1}\right)=\left(\hat{a}^{\dagger}\right)^{\left|\Gamma_{1}^{+}\right|} \hat{a}^{\left|\Gamma_{1}^{-}\right|}$(resp., $\left.\psi\left(\Gamma_{2}\right)=\left(\hat{a}^{\dagger}\right)^{\left|\Gamma_{2}^{+}\right|} \hat{a}^{\left|\Gamma_{2}^{-}\right|}\right)$. The product $\psi\left(\Gamma_{2}\right) \psi\left(\Gamma_{1}\right)$ is given by

$$
\psi\left(\Gamma_{2}\right) \psi\left(\Gamma_{1}\right)=\sum_{i=0}^{\min \left(\left|\Gamma_{1}^{+}\right|,\left|\Gamma_{2}^{-}\right|\right.}\left(\begin{array}{c}
\left|\Gamma_{2}^{-}\right| \\
i
\end{array}\right)\left(\begin{array}{c}
\left|\Gamma_{1}^{+}\right| \\
i
\end{array}\right) i !\left(\hat{a}^{\dagger}\right)^{\left|\Gamma_{1}^{+}\right|+\left|\Gamma_{2}^{+}\right|-i} \hat{a}^{\left|\Gamma_{1}^{-}\right|+\left|\Gamma_{2}^{-}\right|-i}
$$

On the other hand, building the partial product of $\Gamma_{1}$ and $\Gamma_{2}$ where exactly $i$ edges are matched, the resulting graph will have $\left|\Gamma_{1}^{+}\right|+\left|\Gamma_{2}^{+}\right|-i$ outgoing and $\left|\Gamma_{1}^{-}\right|+\left|\Gamma_{2}^{-}\right|-i$ ingoing edges, and will be mapped by $\psi$ to $\left(\hat{a}^{\dagger}\right)^{\left|\Gamma_{1}^{+}\right|+\left|\Gamma_{2}^{+}\right|-i} \hat{a}^{\left|\Gamma_{1}^{-}\right|+\left|\Gamma_{2}^{-}\right|-i}$. Since in $\Gamma_{1} * \Gamma_{2}$ we have to sum over all $i$, and for each $i$ there are $\left(\begin{array}{c}\left|\Gamma_{2}^{-}\right| \\ i\end{array}\right)\left(\begin{array}{c}\left|\Gamma_{1}^{+}\right| \\ i\end{array}\right) i$ ! possibilities, we see that the right-hand side of the formula equals $\psi\left(\Gamma_{1} * \Gamma_{2}\right)$, see [21] for more details. More recently, Bousbaa et al. [23] introduced another Hopf algebra $\mathcal{B}$ of combinatorial objects (" $B$-diagrams") in a slightly different fashion. The $B$-diagrams have the same number of inputs and outputs, but the vertices are labelled. One has an analogue of the map $\psi$ above and the structure constants $\left(\begin{array}{c}q \\ i\end{array}\right)\left(\begin{array}{c}k \\ i\end{array}\right) i$ ! have also a combinatorial interpretation as the number of possible compositions of certain $B$-diagrams. It was shown in [23] that one can recognize two well-known combinatorial Hopf subalgebras of $\mathcal{B}$ : WSym - the algebra of word symmetric functions indexed by set partitions - and BWSym - the algebra of biword symmetric functions indexed by set partitions into lists. These two Hopf algebras were discussed recently also in [1,27].

Let us remark that the considerations of Blasiak et al. [21] were extended by Behr et al. [11] to the case of socalled rule diagram algebras that present graph rewriting rules and their composites. Behr and Sobociński [13] applied this formalism to certain adhesive categories, and Behr et al. [10] applied it to stochastic rewriting systems for graphs.

\section{Normal ordering in the $n$-th Weyl algebra (the multi- mode case)}

In this section, we describe some recent results concerning normal ordering in the multi-mode Weyl algebra $A_{n}$. In Section 3.1, a combinatorial interpretation for the associated normal ordering coefficients is given. In Section 3.2, we mention some other recent work related to normal ordering in $A_{n}$. Since this has mainly an algebraic or analytic (but not combinatoric) flavour we will be brief.

\subsection{Digraphs and normal ordering in the $n$-th Weyl algebra}

In this section, we describe a combinatorial interpretation for normal ordering in the $n$-th Weyl algebra $A_{n}$ given by Dzhumadil'daev and Yeliussizov [50,51]. First, we recall some definitions and notations from [50]. The $n$-th Weyl algebra (over the field $\mathbb{K} \in\{\mathbb{R}, \mathbb{C}\}$ ) is generated by $\left\{X_{i}, D_{i}\right\}_{1 \leq i \leq n}$ satisfying for $1 \leq i, j \leq n$ the commutation relations

$$
D_{i} X_{j}=X_{j} D_{i}+\delta_{i, j}, \quad X_{i} X_{j}=X_{j} X_{i}, \quad D_{i} D_{j}=D_{j} D_{i},
$$

where $\delta_{i, j}$ is the Kronecker symbol. Using multi-index notation, the elements $X^{\alpha} D^{\beta}=X_{1}^{\alpha_{1}} \cdots X_{n}^{\alpha_{n}} D_{1}^{\beta_{1}} \cdots D_{n}^{\beta_{n}}$ with $\alpha=\left(\alpha_{1}, \ldots, \alpha_{n}\right), \beta=\left(\beta_{1}, \ldots, \beta_{n}\right) \in \mathbb{N}_{0}^{n}$ are called monomials. Define the length $\ell\left(X^{\alpha} D^{\beta}\right)$ and weight $\omega\left(X^{\alpha} D^{\beta}\right)$ by

$$
\ell\left(X^{\alpha} D^{\beta}\right)=\sum_{i=1}^{n}\left(\alpha_{i}-\beta_{i}\right), \omega\left(X^{\alpha} D^{\beta}\right)=\left(\alpha_{1}-\beta_{1}, \ldots, \alpha_{n}-\beta_{n}\right) .
$$

We will also write monomials in the form $X_{i_{1}} \cdots X_{i_{r}} D_{j_{1}} \cdots D_{j_{s}}$ with $i_{r}, j_{s} \in[n]$. The monomials form a vector space basis of $A_{n}$. When an element $w \in A_{n}$ is expressed as linear combination

$$
w=\sum_{\alpha, \beta} c_{w}(\alpha, \beta) X^{\alpha} D^{\beta}
$$


we say it is in normal ordered form with normal ordering coefficients $c_{w}(\alpha, \beta)$. The order of $w$ is defined by $\operatorname{ord}(w)=\max _{c_{w}(\alpha, \beta) \neq 0}|\beta|$, where $|\beta|=\beta_{1}+\cdots+\beta_{n}$. Note that $\operatorname{ord}\left(w_{1} w_{2}\right)=\operatorname{ord}\left(w_{1}\right)+\operatorname{ord}\left(w_{2}\right)$. We define the following subspaces of $A_{n}$,

$$
A_{n}^{(0)}=\bigoplus_{i \geq 1} A_{n}^{(i, i)}, \quad A_{n}^{(p, q)}=\left\langle X^{\alpha} D^{\beta}|| \alpha|=p,| \beta \mid=q\right\rangle,
$$

The subspace $A_{n}^{(0)}$ formed by elements of length 0 is in fact a subalgebra. Note that, in principle, normal ordering in $A_{n}$ can be reduced to normal ordering in $A_{1}$ since the elements with different index commute, see (48). In other words, the normal ordering coefficients $c_{w}(\alpha, \beta)$ in (49) can be determined from those in $A_{1}$. In [51, Remark 6.1] where this was mentioned, the example $X_{1} X_{2} D_{1} D_{3} X_{2} X_{3} D_{1} D_{3} X_{1} D_{2}=\left(X_{1} D_{1}^{2} X_{1}\right)\left(X_{2}^{2} D_{2}\right)\left(D_{3} X_{3} D_{3}\right)$ was given for illustration.

Example 3.1. Let $n=3$ and consider the monomials $w_{1}=X_{1} D_{1}, w_{2}=X_{2} D_{3}, w_{3}=X_{2} D_{1}, w_{4}=X_{3} D_{3}, w_{5}=$ $X_{1} D_{2}$ (this is [50, Example 2] with typo in $w_{4}$ corrected). Then one has

$$
w_{1} w_{2} w_{3} w_{4} w_{5}=2 X_{1} X_{2}^{2} D_{1} D_{2} D_{3}+2 X_{1} X_{2}^{2} X_{3} D_{1} D_{2} D_{3}^{2}+X_{1}^{2} X_{2}^{2} D_{1}^{2} D_{2} D_{3}+X_{1}^{2} X_{2}^{2} X_{3} D_{1}^{2} D_{2} D_{3}^{2} .
$$

Now, we want to describe the combinatorial description for the normal ordering coefficients following [50]. Let $G=(V, E)$ be a digraph (with possible multiple edges and cycles). Let us suppose the edges of $G$ are labelled by $m$ indices, $E=\left\{e_{1}, \ldots, e_{m}\right\}$. We consider $k$-decompositions of $G$ with edge-disjoint increasing paths. This means we partition the edge set $E$ with $k$ paths, $E=P_{1} \cup \cdots \cup P_{k}$ such that the edge labels increase along every path $P_{i}=e_{i_{1}} \cdots e_{i_{s}}$, i.e., $i_{1}<\cdots<i_{s}$. Such a $k$-decomposition is also called principal. In the following we suppose that the vertex set of $G$ is given by $[n]$.

Example 3.2. Let $n=3$ and consider the graph $G=\left(V=\{1,2,3\}, E=\left\{e_{1}=(1,1), e_{2}=(2,3), e_{3}=\right.\right.$ $\left.\left.(2,1), e_{4}=(3,3), e_{5}=(1,2)\right\}\right)$, see Figure 6 . It has no $k$-decomposition for $k=1,2$. For $k=5$, it has the trivial 5-decomposition $e_{1} \cup e_{2} \cup e_{3} \cup e_{4} \cup e_{5}$. It has two 3-decompositions $\left(e_{1} e_{5} \cup e_{2} e_{4} \cup e_{3}, e_{1} \cup e_{2} e_{4} \cup e_{3} e_{5}\right)$ and three 2-decompositions $\left(e_{1} \cup e_{2} \cup e_{3} e_{5} \cup e_{4}, e_{1} e_{5} \cup e_{2} \cup e_{3} \cup e_{4}, e_{1} \cup e_{2} e_{4} \cup e_{3} \cup e_{5}\right)$.

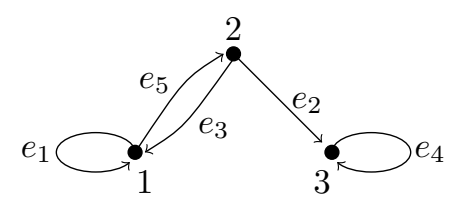

Figure 6: The digraph of Example 3.2.

Example 3.3. Let $G_{m}$ consist of one vertex $(V=\{1\})$ and $m$ labelled loops $(1,1)$. Then a principal decomposition corresponds to a set partition of $[m]$. The number of $k$-decompositions of $G_{m}$ is given by $S(m, k)$.

For a given digraph $G$, let in(i), out(i) $(i \in V)$ denote the numbers of incoming and outgoing edges, respectively. We then introduce the multisets

$$
V_{\text {out }}(G)=\left\{1^{\text {out }(1)}, \ldots, n^{\text {out }(\mathrm{n})}\right\}, \quad V_{\text {in }}(G)=\left\{1^{\text {in }(1)}, \ldots, n^{\text {in(n) }}\right\}, \quad M_{\text {out }}(G)=\left\{I \mid I \subseteq V_{\text {out }}(G)\right\} .
$$

The difference $A-X$ of multisets means that we eliminate from $A$ as many copies of elements as $X$ has. For example, $\left\{1^{3}, 2^{2}, 3,4^{3}\right\}-\left\{1^{2}, 2,4\right\}=\left\{1,2,3,4^{2}\right\} . A \uplus X$ is a merge of multisets, e.g., $\left\{1^{2}, 2,4^{2}\right\} \uplus\left\{1,2^{2}, 3\right\}=$ $\left\{1^{3}, 2^{3}, 3,4^{2}\right\}$. If we have for a given $k$-decomposition $\delta$ the sources $I$, then the corresponding sinks are given by $J(I) \equiv J(\delta, I)=V_{\text {in }}(G) \uplus I-V_{\text {out }}(G)$.

Example 3.4. Consider the graph $G$ from Example 3.2 and the 3-decomposition $e_{1} e_{5} \cup e_{2} e_{4} \cup e_{3}$. The edge $e_{1} e_{5}$ (resp., $e_{2} e_{4}, e_{3}$ ) has source 1 (resp., 2, 2) and sink 2 (resp., 3,1). Thus, $I=\{1,2,2\}$ and $J(I)=\{1,2,3\}$. On the other hand, using $V_{\text {in }}(G)=\left\{1^{2}, 2,3^{2}\right\}, V_{\text {out }}(G)=\left\{1^{2}, 2^{2}, 3\right\}$, we calculate $\left\{1^{2}, 2,3^{2}\right\} \uplus\{1,2,2\}-\left\{1^{2}, 2^{2}, 3\right\}=$ $\{1,2,3\}$, as it should be.

Definition 3.1. [50] The $G$-Stirling function $S_{G}: M_{\text {out }}(G) \rightarrow \mathbb{N}_{0}$ is defined by letting $S_{G}(I)$ be the number of principal decompositions of $G$ with sources $I$. 
For example, if $G_{m}$ is the digraph from Example 3.3, then $S_{G_{m}}(I)=S(m, k)$ if $|I|=k$. Several useful properties of $S_{G}$ were derived in [50], and it was also remarked that $S_{G}(I)$ is different from the graphical Stirling numbers introduced in Definition 2.2. Before returning to the $n$-th Weyl algebra $A_{n}$, we need one further notion. A block (or $p$-block, if $p$ is specified) of a digraph $G$ is a distinguished set of edges $\left\{e_{1}, \ldots, e_{p}\right\}$. If a digraph is built from several (disjoint) blocks, then we require that the edges of each block must lie in distinct paths.

To any monomial $w=X_{i_{1}} \cdots X_{i_{p}} D_{j_{1}} \cdots D_{j_{p}} \in A_{n}^{(0)}$ we associate a $p$-block of a graph in the following way: $\operatorname{block}(w)=\left\{\left(i_{1}, j_{1}\right), \ldots,\left(i_{p}, j_{p}\right)\right\}$. Now, we can state the following result.

Theorem 3.1. [50, Theorem 3.1] Let $w_{1}, \ldots, w_{m} \in A_{n}^{(0)}$. Then one has

$$
w_{1} \cdots w_{m}=\sum_{I \subseteq V_{\text {out }}(G)} S_{G}(I) \prod_{i \in I} X_{i} \prod_{j \in J(I)} D_{j},
$$

where the digraph $G$ with $n$ vertices is built from the $m$ blocks block $\left(w_{1}\right), \ldots, \operatorname{block}\left(w_{m}\right)$ so that the indices of edges increase with respect to the order of blocks, and $J(I)=V_{\mathrm{in}}(G) \uplus I-V_{\text {out }}(G)$.

Example 3.5. Let us return to Example 3.1. To the monomials $w_{1}, \ldots, w_{5}$ we associate the 1 -blocks block $\left(w_{1}\right)=$ $\{(1,1)\}, \operatorname{block}\left(w_{2}\right)=\{(2,3)\}, \operatorname{block}\left(w_{3}\right)=\{(2,1)\}, \operatorname{block}\left(w_{4}\right)=\{(3,3)\}, \operatorname{block}\left(w_{5}\right)=\{(1,2)\}$, and we recognize the resulting digraph $G$ as the one from Example 3.2, see Figure 6. In Table 1, all principal decompositions are given. According to Theorem 3.1, the first line of Table 1 gives a summand $2 X_{1} X_{2}^{2} D_{1} D_{2} D_{3}$, the second line $2 X_{1} X_{2}^{2} X_{3} D_{1} D_{2} D_{3}^{2}$, and so on. In total, we obtain from Theorem 3.1 for $w_{1} \cdots w_{5}$ the expression

$$
2 X_{1} X_{2}^{2} D_{1} D_{2} D_{3}+2 X_{1} X_{2}^{2} X_{3} D_{1} D_{2} D_{3}^{2}+X_{1}^{2} X_{2}^{2} D_{1}^{2} D_{2} D_{3}+X_{1}^{2} X_{2}^{2} X_{3} D_{1}^{2} D_{2} D_{3}^{2},
$$

which is exactly the result obtained in Example 3.1 by hand.

\begin{tabular}{llll}
$I$ & $J(I)$ & Principal decompositions & $S_{G}(I)$ \\
\hline$\{1,2,2\}$ & $\{1,2,3\}$ & $e_{1} e_{5} \cup e_{2} e_{4} \cup e_{3}, e_{1} \cup e_{2} e_{4} \cup e_{3} e_{5}$ & 2 \\
$\{1,2,2,3\}$ & $\{1,2,3,3\}$ & $e_{1} \cup e_{2} \cup e_{3} e_{5} \cup e_{4}, e_{1} e_{5} \cup e_{2} \cup e_{3} \cup e_{4}$ & 2 \\
$\{1,1,2,2\}$ & $\{1,1,2,3\}$ & $e_{1} \cup e_{2} e_{4} \cup e_{3} \cup e_{5}$ & 1 \\
$\{1,1,2,2,3\}$ & $\{1,1,2,3,3\}$ & $e_{1} \cup e_{2} \cup e_{3} \cup e_{4} \cup e_{5}$ & 1
\end{tabular}

Table 1: All principal decompositions of the digraph from Figure 6.

For $n=1$, one has $w=X D$, so to $w^{m}=(X D)^{m}$ corresponds the digraph $G_{m}$ from Example 3.3 according to Theorem 3.1, and one recovers the expression $(X D)^{m}=\sum_{k=1}^{m} S(m, k) X^{k} D^{k}$ [50, Corollary 3.4].

Problem 3.1. The G-Stirling function is a generalization of the Stirling numbers of the second kind to graphs. As mentioned above, it is different from the graphical Stirling number $S(G, k)$ introduced in Definition 2.2 (which is also a generalization of the Stirling numbers of the second kind). For $S(G, k)$, a q-analogue has been introduced by Kereskényiné Balogh and Schlosser [82]. Furthermore, Barghi [7] has extended the Stirling numbers of the first kind to graphs (see also Eu et al. [56], for a brief description see Section 2.1.5, and Galvin and Pacurar [60]). Thus, one should find in a similar fashion a q-analogue of the G-Stirling function and an analogue extending the Stirling numbers of the first kind.

Remark 3.1. As discussed by Dzhumadil'daev and Yeliussizov [51, Section 6.1], the assumption $w_{i} \in A_{n}^{(0)}$ in Theorem 3.1 is not too restrictive since we can always add new variables to satisfy it. For $n=1$, the element $X^{r} D^{s}$ is not an element in $A_{1}^{(0)}$ if $r \neq s$. If $r>s$ (resp., $s>r$ ), we add $r-s$ (resp., $\left.s-r\right)$ new variables $D_{2}$ (resp., $X_{2}$ ) and the resulting monomial belongs to $A_{2}^{(0)}$. For example, $X^{3} D^{7}$ is transformed to $X_{2}^{4} X_{1}^{3} D_{1}^{7}$. The new variables commute with all other variables, so can be freely moved in the normal ordering expansion. The same argument can be used in the case of arbitrary $n$.

Dzhumadil'daev and Yeliussizov [51, Section 6] introduced for $n=1$ certain $\lambda$-Stirling numbers by considering an analogue of the graph $G_{m}$ considered in Example 3.3. For this, consider the graph with $n=1$ vertex and 
suppose it is built from blocks of loops $(1,1)$ of $\lambda_{1}, \lambda_{2}, \ldots$ edges. The principal decompositions for this model require that the edges within one block cannot lie on the same walk. Thus, this setting corresponds to a partition of the set $[m]$ ( $m$ is the total number of edges) where the first $\lambda_{1}$ elements are in distinct subsets, the next $\lambda_{2}$ elements are also in distinct subsets, and so on. The coefficients $S_{G}(I)$ - also called $\lambda$-Stirling numbers $S_{\lambda}(m, k)$ in [51] - are a generalization of $S(n, k)$ taking into account the restrictions on the partitions. As the authors mentioned, these $\lambda$-Stirling numbers are exactly the $\left(r_{1}, \ldots, r_{p}\right)$-Stirling numbers of the second kind introduced by Mihoubi and Maamra [107].

\subsection{Some other developments concerning the multi-mode case}

In this section, some other developments concerning normal ordering in the multi-mode case are mentioned, mostly as a guide to the literature.

1. Accardi and Boukas [3] considered the multi-mode (or, multidimensional) Schrödinger algebra and their aim was to split the exponential of an arbitrary sum of the generators into a product of normal ordered exponentials. A very simple and well-known example is the following: Let $U$ and $V$ satisfy the relation $U V=V U+I$ of the Weyl algebra $A_{1}$. Then one has the disentanglement formula (or splitting formula) $e^{\lambda V+\mu U}=e^{\lambda \mu / 2} e^{\lambda V} e^{\mu U}$ (see, e.g., the discussion in [98]). Using the more physical notation $U \mapsto \hat{a}, V \mapsto \hat{a}^{\dagger}$, one has $\hat{a} \hat{a}^{\dagger}-\hat{a}^{\dagger} \hat{a}=1$, and the splitting formula reads

$$
e^{\lambda \hat{a}^{\dagger}+\mu \hat{a}}=e^{\lambda \mu / 2} e^{\lambda \hat{a}^{\dagger}} e^{\mu \hat{a}} .
$$

To follow the notation of [3], the 1-mode Heisenberg algebra heir(1) is the 3-dimensionsal *-Lie algebra with generators $\left\{1, \hat{a}, \hat{a}^{\dagger}\right\}$, where 1 is a central element and the only nonvanishing commutator is given by $\hat{a} \hat{a}^{\dagger}-\hat{a}^{\dagger} \hat{a}=1$, together with $\left(\hat{a}^{\dagger}\right)^{*}=\hat{a}$ and $1^{*}=1$. The universal enveloping algebra of $\mathfrak{h e i s}(1)$ is denoted by $\mathcal{P}\left(\hat{a}^{\dagger}, \hat{a}\right)$ and can be defined with the polynomial algebra in $\hat{a}^{\dagger}, \hat{a}$. One can choose as generators $B_{k}^{n}=\left(\hat{a}^{\dagger}\right)^{n} \hat{a}^{k}+\delta_{n, k} \frac{1}{2}$, satisfying $\left(B_{k}^{n}\right)^{*}=B_{n}^{k}$. The 1-mode Schrödinger algebra $\mathfrak{S c h r o d}(1)$ is the $*$-Lie sub-algebra of $\mathcal{P}\left(\hat{a}^{\dagger}, \hat{a}\right)$ generated by $\left\{1, \hat{a}, \hat{a}^{2}, \hat{a}^{\dagger},\left(\hat{a}^{\dagger}\right)^{2}, \hat{a}^{\dagger} \hat{a}+\frac{1}{2}\right\}$, or, using the just introduced notation, $\left\{B_{0}^{0}, B_{1}^{0}, B_{2}^{0}, B_{0}^{1}, B_{0}^{2}, B_{1}^{1}\right\}$. One has the commutation relation

$$
B_{k}^{n} B_{K}^{N}-B_{K}^{N} B_{k}^{n}=(k N-n K) B_{k+K-1}^{n+N-1},
$$

where $n, k, N, K \in\{0,1,2\}$ with $n+k \leq 2$ and $N+K \leq 2$. (Unfortunately, there is a small typo in the corresponding [3, Eq. (2.2)] which is repeated at several places. Thus, one should consult the former paper by Accardi and Boukas [2] for this.) A linear combination of the nontrivial generators of $\mathfrak{S c h r o d}(1)$ can then be written as $\mathcal{B}=c_{2,0} B_{0}^{2}+c_{1,0} B_{0}^{1}+c_{1,1} B_{1}^{1}+c_{0,1} B_{1}^{0}+c_{0,2} B_{2}^{0}$ where $c_{n, k} \in \mathbb{C}$. The aim now is to split the group element $e^{\mathcal{B}}$ - in analogy to (50) - as a product of normal ordered exponentials, i.e., in a form like $e^{c_{2,0} B_{0}^{2}+c_{1,0} B_{0}^{1}} e^{c_{1,1} B_{1}^{1}} e^{c_{0,1} B_{1}^{0}+c_{0,2} B_{2}^{0}}$. In this 1-dimensional case, it was derived by Accardi and Boukas [2, Lemma 3.3] (see also [3, Corollary 3.7]). In the recent paper [3], these considerations were extended to the multi-mode case $\mathfrak{S c h r o d}(d)$. Here we have $d$ pairs of elements $\hat{a}_{i}^{\dagger}, \hat{a}_{i}, i=1, \ldots, d$, together with the central element 1 , satisfying

$$
\hat{a}_{i} \hat{a}_{j}^{\dagger}-\hat{a}_{j}^{\dagger} \hat{a}_{i}=\delta_{i, j} 1, \quad \hat{a}_{i}^{\dagger} \hat{a}_{j}^{\dagger}=\hat{a}_{j}^{\dagger} \hat{a}_{i}^{\dagger}, \quad \hat{a}_{i} \hat{a}_{j}=\hat{a}_{j} \hat{a}_{i},
$$

compare (48). The $d$-mode Schrödinger algebra $\mathfrak{S c h r o d}(d)$ is the $*$-Lie algebra generated by the elements $\left\{1, \hat{a}_{h}, \hat{a}_{i} \hat{a}_{j}, \hat{a}_{k}^{\dagger}, \hat{a}_{i}^{\dagger} \hat{a}_{j}^{\dagger}, \hat{a}_{h}^{\dagger} \hat{a}_{k}+\frac{1}{2} \mid h, i, j, k=1, \ldots, d, i \leq j\right\}$. One has a commutation relation similar to (51), and the disentanglement problem faces up in a fashion similar to the case $d=1$, see [3, Theorem 4.3] for a solution of this hard problem. Let us note that Chebotarev et al. [36,37] also considered this problem. Closely related problems were treated by Teretenkov in a series of papers, see his review [130] where also some useful commutator formulas and many references can be found.

2. Let us consider the $n$-mode boson where we have $n$ pairs of generators $\hat{a}_{i}^{\dagger}, \hat{a}_{i}, i=1, \ldots, n$, satisfying the canonical commutation relations (52). Following the notation of Chebotarev et al. [36,37], we can write a Hamiltonian $\mathcal{H}$ which is at most quadratic in the generators as

$$
\mathcal{H}=\frac{i}{2}\left(\hat{a}^{\dagger}, A \hat{a}^{\dagger}\right)-\frac{i}{2}(\hat{a}, \bar{A} \hat{a})+\left(\hat{a}^{\dagger}, B \hat{a}\right)+i\left(\hat{a}^{\dagger}, h\right)-i(\hat{a}, \bar{h}),
$$


where $A=A^{t} \in \mathbb{C}^{n, n}$ is a symmetric matrix, $B=B^{*}=\bar{B}^{t} \in \mathbb{C}^{n, n}$ is a Hermitian matrix, $h \in \mathbb{C}^{n}$, and, by a slight abuse of notation, $\hat{a}^{\dagger}=\left(\hat{a}_{1}^{\dagger}, \ldots, \hat{a}_{n}^{\dagger}\right)$ and $\hat{a}=\left(\hat{a}_{1}, \ldots, \hat{a}_{n}\right)$. Note that for $n=1, \mathcal{H}=\mathcal{B}$ from the last point. Of interest in all references mentioned there is an explicit expression - or a normal ordered form - for the unitary group $U_{t}=e^{i \mathcal{H} t}$. Brádler [24] suggested recently another method to calculate the unitary evolution operator. He assumed that the Hamiltonian is a Hermitian operator which can be written as $\mathcal{H}=\nu \mathcal{A}^{\dagger}+\bar{\nu} \mathcal{A}$ where $\nu \in \mathbb{C}$ and the operator $\mathcal{A}$ is a function of one or more boson creation and annihilation operators, $\hat{a}_{i}^{\dagger}$ and $\hat{a}_{i}$ satisfying (52). Letting $\nu=s+i r$, he restricted to $V=e^{r\left(\mathcal{A}^{\dagger}-\mathcal{A}\right)}$, and, by arguing that in many cases one makes a Taylor expansion around $r=0$, focussed on the powers $\left(\mathcal{A}^{\dagger} \pm \mathcal{A}\right)^{k}$. The main idea of [24] is to consider the expansion in a weak sense, i.e., when acting on a "state of interest" $\psi,\left(\mathcal{A}^{\dagger} \pm \mathcal{A}\right)^{k} \psi$ (and to avoid calculating $\left(\mathcal{A}^{\dagger} \pm \mathcal{A}\right)^{k}$ itself). For the following, it is assumed that $A \psi=0$. To avoid sign complications, we consider $\left(\mathcal{A}^{\dagger}+\mathcal{A}\right)^{k}$ and write [24, Eq. (9)]

$$
\left(\mathcal{A}^{\dagger}+\mathcal{A}\right)^{k}=\sum_{m=0}^{k} \sum_{|S|=1}^{\left(\begin{array}{c}
k \\
m
\end{array}\right)}(\mathcal{A})^{m_{1}}\left(\mathcal{A}^{\dagger}\right)^{m_{2}} \cdots(\mathcal{A})^{m_{2 J-1}}\left(\mathcal{A}^{\dagger}\right)^{m_{2 J}},
$$

where $S=\left\{m_{1}, \ldots, m_{2 J} ; m_{j} \geq 0, \sum_{j=1}^{2 J} m_{k}=k, \sum_{j=1}^{J} m_{2 J}=m\right\}$, compare with (62). This sum contains $2^{k}$ summands, but at least half of all the products vanish upon acting on $\psi$ because there are more operators $\mathcal{A}$ than $\mathcal{A}^{\dagger}$ in it. But more is true: From the way a word $\omega=(\mathcal{A})^{m_{1}}\left(\mathcal{A}^{\dagger}\right)^{m_{2}} \cdots(\mathcal{A})^{m_{2 J-1}}\left(\mathcal{A}^{\dagger}\right)^{m_{2 J}}$ acts on $\psi$, we see that it vanishes if $m_{2 J-1}>m_{2 J}, m_{2 J-3}>m_{2 J-2}+m_{2 J}-m_{2 J-1}$, and so on. Following [24], we can represent such a word as a path in $\mathbb{Z}^{2}$ by associating to the operators the steps

$$
\mathcal{A} \longleftrightarrow D=(1,-1), \quad \mathcal{A}^{\dagger} \longleftrightarrow U=(1,1)
$$

A word $\omega$ is read from right to left, i.e., $\omega \longleftrightarrow \Gamma_{\omega}=U^{m_{2 J}} D^{m_{2 J-1}} \cdots U^{m_{2}} D^{m_{1}}$ (but the steps of the path are read as usual from left to right, i.e., starting with $\left.U^{m_{2 J}}\right)$. Now, the above condition that the action of $\omega$ on $\psi$ does not vanish translates into the condition that the associated path $\Gamma_{\omega}$ never goes below the $x$-axis. Following [24, Section 2], we let $\mathfrak{D}\left(k, \delta_{1}, \delta_{2}\right)$ be the set of generalized Dyck paths starting at $\left(0, \delta_{1}\right)$, ending at $\left(k, \delta_{2}\right)$, having steps $U=(1,1)$ and $D=(1,-1)$ and always staying in the positive quadrant including the $x$-axis. Note that $\mathfrak{D}(k, 0,0)$ (with $k$ even) is the set of conventional Dyck paths. Thus, the summands in (53) which do not vanish upon acting on $\psi$ correspond to generalized Dyck paths $\mathfrak{D}\left(k, 0, \delta_{2}\right)$. This association was discussed from several points of view by Brádler [24]. Before concluding this point, let us recall that we already used a similar correspondence at other places (see, e.g., Section 2.1.4 where we used an equivalent description for Dyck paths).

3. Finkelshtein et al. [57] considered recently an "infinite dimensional" generalization of the Stirling numbers in terms of Stirling operators in spatial combinatorics. Unfortunately, it would lead too far away to introduce the precise setting, but let us mention that the basis for all considerations is a locally compact Polish space $X$, and that when $X$ consists of one point, the conventional objects (e.g., Stirling numbers and Bell numbers) and formulas (e.g., (3) or the Dobiński formula) are recovered. Furthermore, certain normal ordering problems in the context of spatial combinatorics were considered in [57].

\section{Normal ordering in new algebras}

In this section, recent research on normal ordering symbols $U$ and $V$ satisfying some new commutation relation is described. Many of these commutation relations are modifications of the commutation relation $U V=q V U$ of the quantum plane (Example 1.2) or of $U V-q V U=I$, the commutation relation of the $q$-deformed Weyl algebra (Example 1.3). The two examples presented in Sections 4.7 and 4.8 have three or more generators.

\subsection{Algebras defined by a linearly twisted commutation relation}

Cantuba [31] considered normal ordering in an algebra defined by a linearly twisted commutation relation. Let $\mathbb{K}\langle U, V\rangle$ be the unital associative algebra over a field $\mathbb{K}$ generated by two elements $U, V$. The multiplicative 
product is given by concatenation, and we also use $U^{0}=V^{0}=I$. Given a function $\sigma: \mathbb{K}\langle U, V\rangle \rightarrow \mathbb{K}\langle U, V\rangle$, denote by $\mathcal{I}_{\sigma}$ the two sided ideal of $\mathbb{K}\langle U, V\rangle$ generated by $U V-\sigma(V U)$. The defining relation $U V=\sigma(V U)$ of the quotient algebra $\mathbb{K}\langle U, V\rangle / \mathcal{I}_{\sigma}$ is called $\sigma$-twisted commutation relation. If there exist $m, b \in \mathbb{K}$ with $q \neq 0$ such that $\sigma(X)=q X+b I$, then the commutation relation $U V=\sigma(V U)$ is called linearly twisted. The associated algebra $\mathbb{K}\langle U, V\rangle / \mathcal{I}_{\sigma}$ is then a variant of the $q$-deformed Weyl algebra over $\mathbb{K}$, see Example 1.3. To study its properties, Cantuba [31] switched to an isomorphic algebra in three generators by adding the commutator. More precisely, consider $\mathbb{K}\langle U, V, C\rangle$ to be the associative unital algebra over a field $\mathbb{K}$ generated by the elements $U, V, C$ and denote by $\mathcal{J}_{\sigma}$ the ideal generated by $U V-\sigma(V U)$ and $C-U V+V U$. Then there exists an algebra isomorphism $\mathbb{K}\langle U, V\rangle / \mathcal{I}_{\sigma} \rightarrow \mathbb{K}\langle U, V, C\rangle / \mathcal{J}_{\sigma}$ such that $U \mapsto U, V \mapsto V, U V-V U \mapsto C$. In these two algebras several reordering formulas were derived [31].

\subsection{A slight generalization of the $q$-deformed Weyl algebra}

Cantuba and Merciales [32] continued the study of Section 4.1 in the following way. The $q$-deformed Weyl algebra $A_{1}(q)$ over a field $\mathbb{K}$ with a fixed $q \in \mathbb{K}$ is the unital associative algebra over $\mathbb{K}$ with generators $U, V$ and a relation which asserts that $U V-q V U$ is the multiplicative identity in $A_{1}(q)$, see Example 1.3. In [32], $A_{1}(q)$ is extended into an algebra $\mathcal{R}(q)$ defined by generators $U, V$ and a relation which asserts that $U V-q V U$ is central in $\mathcal{R}(q)$. Thus, $U(U V-q V U)=(U V-q V U) U$ as well as $V(U V-q V U)=(U V-q V U) V$. Introducing for the central $q$-commutator a new letter, $\gamma=U V-q V U$, one can show [32, Lemma 3.1] that $\mathcal{R}(q)$ has a presentation by generators $U, V, \gamma$ with relations

$$
\gamma=U V-q V U, \quad U \gamma=\gamma U, \quad V \gamma=\gamma V .
$$

Furthermore, the elements $\gamma^{h} V^{m} U^{n}\left(h, m, n \in \mathbb{N}_{0}\right)$ form a basis for $\mathcal{R}(q)$ [32, Theorem 3.2]. We say an element in $\mathcal{R}(q)$ is in normal ordered form if it is a (finite) sum of such basis elements (with coefficients in $\mathbb{K}$ ). Many reordering formulas were derived in [32], resembling those of the $q$-deformed Weyl algebra $A_{1}(q)$ (the main difference is that here one has to keep track of the powers of the central element $\gamma$ ).

\subsection{A two-parameter family of generalized q-deformed Weyl algebras}

In Example 1.4, the commutation relation $U V-V U=V^{s}$ was briefly mentioned, see (9). Varvak [131] gave a combinatorial interpretation for the normal ordering coefficients of a word $\omega$ in $U, V$ in terms of the $s$-rook numbers introduced by Goldman and Haglund [61]. At the same time, Burde [28] considered matrices obeying the same commutation relation and derived reordering results. Mansour and Schork [97] considered the algebra $\mathcal{A}_{s ; h \mid q}$ generated by $U$ and $V$ satisfying $U V-q V U=h V^{s}$, where $h \in \mathbb{C} \backslash\{0\}$ and $s \in \mathbb{N}_{0}$. For example, one has the basic normal ordering result [97, Corollary 4.11]

$$
U^{m} V^{n}=\sum_{j=0}^{m}\left(q^{(m-j) n} h^{j}\left(\begin{array}{c}
m \\
j
\end{array}\right)_{q^{s-1}} \prod_{i=0}^{j-1}[n+(s-1) i]_{q}\right) V^{n+j(s-1)} U^{m-j} .
$$

For $q=1$, an equivalent result was shown by Burde [28, Proposition 5.2]. Generalized Stirling numbers $\mathfrak{S}_{s ; h}(n, k \mid q)$ were introduced by Mansour et al. $[100,101]$ as normal ordering coefficients of $(V U)^{n}$. For $q=1$, they are a particular subfamily of the generalized Stirling numbers $S(n, k ; \alpha, \beta, r)$ of Hsu and Shiue [65], see (13). More precisely, one has $\mathfrak{S}_{s ; h}(n, k)=S(n, k ;-h s, h(1-s), 0)$ [101, Theorem 2]. From their definition as normal ordering coefficients one obtains a recurrence relation for the $\mathfrak{S}_{s ; h}(n, k \mid q)$ which allows to consider $s \in \mathbb{R}$. Many properties of these generalized Stirling numbers - as well as those of the associated generalized Bell numbers $\mathfrak{B}_{s ; h}(n \mid q)$ - are collected in [98]. More recently, Celeste et al. [35] introduced a $q$-analogue of the $s$-rook numbers and interpreted the normal ordering coefficients of a word $\omega$ in variables $U, V$ satisfying $U V-q V U=h V^{s}$ in terms of these $q$-deformed $s$-rook numbers (here $s \in \mathbb{N}_{0}$ ). They also gave a new proof of (54) using this combinatorial interpretation [35, Theorem 1]. Furthermore, another combinatorial interpretation was given in [35] in terms of generalized symmetric functions. In a subsequent paper [46], the same authors provided a modification of the rook model of Goldman and Haglund [61] by introducing certain pre-weights for the cells of a board which allows to consider arbitrary $s \in \mathbb{R}$. They also showed an extension of the Spivey 
identity (30) to the generalized Bell numbers $\mathfrak{B}_{s ; h}(n \mid q)$ [46, Corollary 6] (which recovers for $q=1$ a result of Mansour et al. [100, Theorem 5.3]).

Let us consider the two operators $V=X^{\alpha}$ (with $\alpha \in \mathbb{R}$ ) and $U=D=\frac{d}{d x}$ acting on smooth functions $f$. One has $(U V-V U) f=\left(D X^{\alpha}-X^{\alpha} D\right) f=\alpha X^{\alpha-1} f=\alpha V^{\frac{\alpha-1}{\alpha}} f$, or,

$$
U V-V U=\alpha V^{\frac{\alpha-1}{\alpha}} \text {. }
$$

Let us specialize to $\alpha=\frac{1}{\ell}$, with $\ell \in \mathbb{N}$. Here we find $U V-V U=(1 / \ell) V^{1-\ell}$. Thus, normal ordering $(V U)^{n}$ should be closely related to the generalized Stirling numbers $\mathfrak{S}_{1-\ell ; 1 / \ell}(n, k)$. Recall that in Section 2.1.1 we considered Stirling numbers of fractional order $S^{\frac{1}{r}}(n, k), r \in \mathbb{N}$, introduced by Katugampola [77], see Definition 2.1 and the following. Comparing with above, we see that they should be closely related to the generalized Stirling numbers considered here, i.e., $S^{\frac{1}{r}}(n, k) \sim \mathfrak{S}_{1-r ; 1 / r}(n, k)$, and using $\mathfrak{S}_{1-r ; 1 / r}(n, k)=S(n, k ;(r-1) / r, 1,0)$, one expects $S^{\frac{1}{r}}(n, k) \sim S(n, k ;(r-1) / r, 1,0)$.

Problem 4.1. Make these suggested connections between $S^{\frac{1}{r}}(n, k)$ and $\mathfrak{S}_{1-r ; 1 / r}(n, k)$ (or between $S^{\frac{1}{r}}(n, k)$ and $S(n, k ;(r-1) / r, 1,0))$ precise. Use the modified s-rook model with pre-weights [46] to give a combinatorial interpretation for $S^{\frac{1}{r}}(n, k)$.

\subsection{A new $q$-deformed generalized Heisenberg algebra}

Chung [39] introduced a $q$-deformation of a particular family of generalized Heisenberg algebras. We follow the notation of [39] and denote by $\hat{a}^{\dagger}$ and $\hat{a}$ the creation and annihilation operator. The involution of taking adjoints is denoted by $\dagger$, such that $(\hat{a})^{\dagger}=\hat{a}^{\dagger}$ and $\left(\hat{a}^{\dagger}\right)^{\dagger}=\hat{a}$. Furthermore, we assume a self-adjoint Hamiltonian $\hat{H}$ is given as well as an analytic function $f$, the characteristic function. The q-deformed generalized Heisenberg algebra is then defined by the relations

$$
\hat{a} \hat{a}^{\dagger}-q \hat{a}^{\dagger} \hat{a}=f(\hat{H})-q \hat{H}, \quad \hat{H} \hat{a}^{\dagger}=\hat{a}^{\dagger} f(\hat{H}) .
$$

Taking the adjoint of the second relation yields $\hat{a} \hat{H}=f(\hat{H}) \hat{a}$. For example, if $f(\hat{H})=1+q \hat{H}$, the first relation reduces to the commutation relation of the $q$-deformed Weyl algebra $A_{1}(q)$. Several normal ordering results were derived in [39] for the $q$-deformed generalized Heisenberg algebra (and also for special choices of $f$ ). In particular, the $f$-Stirling operator of the second kind $S_{f}(n, k, \hat{H})$ was introduced as normal ordering coefficient for $\left(\hat{a}^{\dagger} \hat{a}\right)^{n}$, i.e., $\left(\hat{a}^{\dagger} \hat{a}\right)^{n}=\sum_{k=0}^{n} \hat{a}^{\dagger} S_{f}(n, k, \hat{H}) \hat{a}$ and several of its properties were derived.

\subsection{The excedance algebra revisited}

Ehrenborg et al. [52, Definition 2.1] introduced the Box polynomials for $m, n \in \mathbb{N}$ by

$$
B_{m, n}(x)=\sum_{\lambda \subseteq m \times n} \prod_{i=1}^{m}\left(x+\lambda_{i}\right),
$$

where the sum is over all partitions $\lambda=\left(n \geq \lambda_{1} \geq \lambda_{2} \geq \cdots \geq \lambda_{m} \geq 0\right)$, i.e., all partitions with $m$ nonnegative parts, each at most $n$. (In other words, the sum is over all partitions $\lambda$ that fit in the $m \times n$ box, hence the name. Later, this set will also be denoted by $\mathcal{P}_{m, n}$ ) If we denote by $\Delta$ the forward difference operator $(\Delta f)(x)=f(x+1)-f(x)$, one has $B_{m, n}(x)=\Delta^{n}\left(x^{m+n}\right) / n$ ! [52, Theorem 2.6]. Writing the difference operator as $\Delta=E-\mathrm{Id}$, where $(E f)(x)=f(x+1)$ is the shift operator, and expanding the binomial $\Delta^{n}=(E-\mathrm{Id})^{n}$ when acting on $x^{m+n}$, one finds the alternative expression $B_{m, n}(x)=\sum_{j=0}^{m}\left(\begin{array}{c}m+n \\ j\end{array}\right) S(m+n-j, n) x^{j}$ [52, Proposition 2.8]. Another interpretation can be given in terms of the excedance algebra defined by Clark and Ehrenborg [41, Definition 1.1]. It is the algebra generated by $U$ and $V$ satisfying the commutation relation

$$
U V-V U=U+V .
$$

(A $q$-deformed version where $U V-q V U=U+V$ was considered in a combinatorial fashion by Corteel et al. [48] and Josuat-Vergès [72] with the Matrix Ansatz. These works were motivated by physical PASEP models where evaluating $(U+V)^{n}$ plays a major role). If $\omega$ is a word in $U, V$ satisfying (55) with $m$ copies of $V$ and $n$ copies of 
$U$, we can write $\omega=\sum_{i, j} c_{i, j}(\omega) V^{i} U^{j}$ with the normal ordering coefficients $c_{i, j}(\omega)$, for $0 \leq i \leq m$ and $0 \leq j \leq n$. An interpretation for $c_{i, j}(\omega)$ in terms of placements of arrows in Ferrers diagrams was given in [41, Theorem 2.1]. Recall that one has in the Weyl algebra $(V U)^{n}=\sum_{k} S(n, k) V^{k} U^{k}$. Writing $(V U)^{n}=V(U V)^{n-1} U$, normal ordering $(V U)^{n}$ is equivalent to normal ordering $(U V)^{n-1}$. If $U, V$ satisfy (55), then the normal ordering coefficients of $(U V)^{m}$ are related to Ghandi polynomials and Genocchi numbers [41]. More generally, let us follow [52] and denote by $E(m, n)$ the sum of all words in $U, V$ with exactly $m$ copies $V$ and $n$ copies $U$. It can be written as $E(m, n)=\sum_{i, j} c_{i, j}^{m, n} V^{i} U^{j}$ with uniquely determined normal ordering coefficients $c_{i, j}^{m, n}$. The box polynomial is then given by $B_{m, n}(x)=\sum_{j=0}^{m} c_{j, n}^{m, n} x^{j}$ [52, Proposition 8.4]. Comparing this to the above expression, one finds $c_{j, n}^{m, n}=\left(\begin{array}{c}m+n \\ j\end{array}\right) S(m+n-j, n)$ [52, Corollary 8.9]. More surprising is the following result: If we let the excedance matrix $M(m, n)$ be the $(m+1) \times(n+1)$ matrix whose $(i, j)$ entry is $c_{i, j}^{m, n}$, then the sum over all entries of $M(m, n)$ is the Eulerian number $A(m+n+1, n+1)$, A008292 in [127], [52, Proposition 8.5]. Let us conclude this section by mentioning that at the end of [52] many open problems were collected.

\subsection{A monomial generalization of the quantum plane}

Recall from Example 1.2 that the quantum plane is characterized by two variables $U, V$ satisfying $U V=q V U$. In the undeformed case, the relations $U V=V^{s} U$ with $s \in \mathbb{N}$ had also been considered, see, e.g., [114]. Mansour and Schork [99] considered the common generalization where $U V=q V^{s} U$ and determined several normal ordering results. For example, for $m, n \in \mathbb{N}_{0}$, one has the following normal ordering result [99, Proposition 2.1],

$$
U^{m} V^{n}=q^{n\left(1+s+\cdots+s^{m-1}\right)} V^{n s^{m}} U^{m},
$$

which one can write compactly as $U^{m} V^{n}=q^{n[m]_{s}} V^{n s^{m}} U^{m}$, using basic numbers $[m]_{s}=1+s+\cdots+s^{m-1}$. This result can be extended to arbitrary words in $U$ and $V$ as follows [99, Theorem 2.4]. Let $k \geq 1$. Then one has, for all $r_{k}, t_{k}, \ldots, r_{1}, t_{1} \in \mathbb{N}_{0}$, the normal ordering result,

$$
U^{r_{k}} V^{t_{k}} \cdots U^{r_{1}} V^{t_{1}}=q^{\sum_{i=1}^{k} \sum_{j=i}^{k} t_{i}\left(1+s+\cdots+s^{r_{j}-1}\right) s^{r_{i}+\cdots+r_{j-1}}} V^{\sum_{i=1}^{k} t_{i} s^{r_{i}+\cdots+r_{k}}} U^{r_{1}+\cdots+r_{k}} .
$$

Problem 4.2. Recall from Example 1.2 that in the case $s=1$ of the quantum plane the exponent of $q$ counts the number of inversions. It would be interesting to find a similar interpretation for the exponent of $q$ in the case of arbitrary s given above. See also Problem 5.1 for a related problem.

\subsection{A polynomial multivariate generalization of the quantum plane}

In the preceding section, a generalized quantum plane was considered. It was defined by the unital associative algebra (over $\mathbb{C}$ ) generated by $U$ and $V$ satisfying $U V=q V^{s} U$. A first step to generalize this situation is to consider instead of a monomial $q V^{s}$ a polynomial $\sigma(V)$ in $V$, i.e., to consider $U V=\sigma(V) U$. Musonda et al. [110] (see also Musonda et al. [111] for more details and proofs) generalized this situation by considering the unital associative algebra $\mathcal{A}_{\sigma_{j}}$ generated by $U_{1}, \ldots, U_{n}, V(n \in \mathbb{N})$ satisfying, for $j=1, \ldots, n$, the commutation relation

$$
U_{j} V=\sigma_{j}(V) U_{j}
$$

where $\sigma_{j}$ are polynomials (and $U_{i} U_{j}=U_{j} U_{i}$ ). Clearly, for $n=1$ and $\sigma(V)=q V^{s}$, one recovers the situation considered in Section 4.6. Choosing instead a polynomial, $\sigma(V)=a_{0}+a_{1} V+\cdots+a_{\ell} V^{\ell}$, one obtains a polynomial generalization of the quantum plane where $U V=\left(\sum_{j=0}^{\ell} a_{j} V^{j}\right) U$. For arbitrary $n$, Musonda et al. [110] determined a host of reordering formulas. For example, if $F$ denotes a polynomial, then one has [110, Theorem 2.1] $U_{j}^{k} F(V)=F\left(\sigma_{j}^{\circ k}(V)\right) U_{j}^{k}$, where $\sigma^{\circ k}$ denotes the $k$-fold composition of the function $\sigma$ with itself. Let us turn to the case $n=2$. Here we have three generators $U_{1}, U_{2}, V$ satisfying $U_{1} V=\sigma(V) U_{1}$ and $U_{2} V=\tau(V) U_{2}$ with polynomials $\sigma, \tau$. It is straightforward to show, for example, the following relations [110, Example 2.4]: $U_{1} V^{l}=(\sigma(V))^{l} U_{1}$ and $U_{2} V^{l}=(\tau(V))^{l} U_{2}, U_{1} F(V)=(F \circ \sigma)(V) U_{1}$ and $U_{2} F(V)=(F \circ \tau)(V) U_{2}$. Let us specialize even further and consider $\sigma(V)=q_{1} V^{s_{1}}$ and $\tau(V)=q_{2} V^{s_{2}}$, i.e.,

$$
U_{1} V=q_{1} V^{s_{1}} U_{1}, \quad U_{2} V=q_{2} V^{s_{2}} U_{2}, \quad U_{1} U_{2}=U_{2} U_{1} .
$$


Applying twice the identity $U^{m} V^{n}=q^{n[m]_{s}} V^{n s^{m}} U^{m}$ from Section 4.6 (commuting $U_{2}$ with $V$ and commuting $U_{1}$ with $V$ ), one obtains [110, Corollary 4.4]

$$
U_{1}^{m_{1}} U_{2}^{m_{2}} V^{n}=q_{1}^{n\left[m_{1}\right]_{s_{1}} s_{2}^{m_{2}}} q_{2}^{n\left[m_{2}\right]_{s_{2}}} V^{n s_{1}^{m_{1}} s_{2}^{m_{2}}} U_{1}^{m_{1}} U_{2}^{m_{2}} .
$$

Note that most examples considered up to now were essentially "one dimensional", i.e., have only one nontrivial commutation relation. The algebra $\mathcal{A}_{\sigma_{j}}$ has $n$ nontrivial commutation relations, but they are, in a certain sense, independent from another. Thus, one should find a graphical representation which shows this fact. To give an idea, we introduce as a first step a commutation graph associated to a set of generators and commutation relations as follows: To each generator we associate a vertex of the graph, and two vertices of the graph are connected by an edge if and only if the two generators do not commute.

Example 4.1. Let us use the notations introduced in Section 2.1.4: $K_{m}$ denotes the complete graph with $m$ vertices ( $K_{1}$ being an isolated vertex), and $n K_{m}$ denotes the disjoint union of $n$ graphs $K_{m}$. Then the commutation graph is given for (see Figure 7):

1. The ring of polynomials in two (resp., $n$ ) commuting variables by $2 K_{1}$ (resp., $n K_{1}$ ).

2. The quantum plane (or its generalization in Section 4.6) by $K_{2}$.

3. The first Weyl algebra $A_{1}$ (or its q-deformation $A_{1}(q)$, or the excedance algebra in Section 4.5) by $K_{2}$.

4. The $n$-th Weyl algebra $A_{n}$ by $n K_{2}$.

5. The algebra $\mathcal{A}_{\sigma_{j}}$ by the star graph $S_{n}$ (the internal node corresponds to $V$, the $n$ leaves to $U_{1}, \ldots, U_{n}$ ).

$$
2 K_{1}
$$
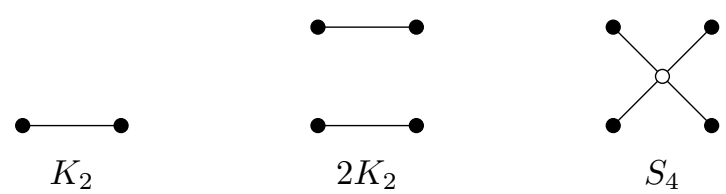

$S_{4}$

Figure 7: Commutation graphs of Example 4.1.

Problem 4.3. Although it captures some aspects of the complexity, the commutation graph can only be a first step: An edge is drawn whenever the associated generators do not commute (implying that all algebras with two non-commuting generators have commutation graph $K_{2}$ ). There is no indication "how far" from being commutative the generators are - and no discrimination whether other generators are involved. Thus, one should refine the construction (e.g., to hypergraphs?) and find good discriminators (e.g., Betti numbers?) for the "commutation complexity". See also Remarks 4.1 and 6.1 for further examples of commutation graphs.

\subsection{An algebra with 3 generators}

In the preceding section, we considered algebras $\mathcal{A}_{\sigma_{j}}$ with generators $U_{1}, \ldots, U_{n}, V$ and commutation relations (56). Musonda et al. [109, Eq. (21.1)] considered a unital associative algebra $\mathcal{A}_{R, J, Q}$ (over $\mathbb{C}$ ) generated by three elements $R, J$ and $Q$ satisfying

$$
Q R-R Q=J, \quad Q J-J Q=-R, \quad R J-J R=0 .
$$

A concrete representation mentioned in [109] is

$$
R(f)(x)=\frac{f(x+i)+f(x-i)}{2}, \quad J(f)(x)=\frac{f(x+i)-f(x-i)}{2 i}, \quad Q(f)(x)=x f(x),
$$

acting on complex functions $f$ (see also Musonda et al. [112] for further aspects of representing $R, J$ and $Q)$. By using the commutation relations, any word in $R, J, Q$ can be brought in normal ordered form, $\omega=$ $\sum_{j, k, l} A_{j, k, l}(\omega) R^{j} J^{k} Q^{l}$ for some normal ordering coefficients $A_{j, k, l}(\omega)$. Since $R$ and $J$ commute, this can be written as $\omega=\sum_{k \geq 0} p_{k}(R, J) Q^{k}$ where the coefficients $p_{k}(R, J)$ are polynomials in $R, J$. In [109] several 
interesting reordering formulas were derived. The first equation of (57) can be written as $[Q, R]=J$, and a small induction shows $\left[Q, R^{m}\right]=m R^{m-1} J, m \in \mathbb{N}[109$, Lemma 21.1]. In a similar fashion, one finds $\left[Q, J^{n}\right]=-n R J^{n-1}, n \in \mathbb{N}[109$, Lemma 21.2]. These two results can be combined to show that for any polynomial $p(R, J)$ in $R$ and $J$ one has [109, Proposition 21.1]

$$
[Q, p(R, J)]=J \frac{\partial p(R, J)}{\partial R}-R \frac{\partial p(R, J)}{\partial J} .
$$

The commutator expressions $\left[Q^{n}, J\right]$ and $\left[Q^{n}, R\right]$ are more cumbersome to determine. Let $S=R+i J$ and $T=R-i J$. Then one has the following observation [109, Proposition 21.8]: The elements $S, T$ and $Q$ satisfy the commutation relations

$$
[Q, S]=-i S, \quad[Q, T]=i T, \quad[S, T]=0,
$$

if and only if $R, J$ and $Q$ satisfy (57). Thus, by this linear transformation of generators one has, in a sense, decoupled the three-dimensional problem $\{R, J, Q\}$ into the two-dimensional problems $\{S, Q\}$ and $\{T, Q\}$. By induction, one infers from the above commutation relations easily $\left[Q, S^{m} T^{n}\right]=(n-m) i S^{m} T^{n}[109$, Proposition 21.9]. This equals $Q S^{m} T^{n}=S^{m} T^{n}(Q+(n-m) i)$. By a further induction, one can show for $k \in \mathbb{N}$ that $Q^{k} S^{m} T^{n}=S^{m} T^{n}(Q+(n-m) i)^{k}[109$, Proposition 21.10].

Remark 4.1. According to Section 4.7, we can associate to $\mathcal{A}_{R, J, Q}$ its commutation graph, the path graph $P_{3}$ on 3 vertices: There are two edges connecting $Q$ with $R$ and $J$, but no edge connecting $R$ and $J$, see (57).

\section{The non-commutative binomial formula}

The binomial formula for non-commuting objects has been considered many times. We mentioned above the $q$-binomial formula (4) for variables $U, V$ satisfying $V U=q U V$ as well as the binomial formula (10) for variables $U, V$ satisfying the commutation relation of the Jordan plane, i.e., $V U-U V=h V^{2}$. For the $q$-deformation of the Jordan plane as well as for the $q$-deformation of the Weyl algebra (and, of course, the Weyl algebra itself) as well as several relatives, like, e.g., the one in [119], analogous binomial formulas are known, see [98]. As was mentioned briefly in Sections 3.2 and 4.5, such binomial formulas often appear in applications. Recently, the study of the binomial formula was considered again and one may, roughly speaking, distinguish two lines of research: A more abstract approach considers rather general situations and discusses possible properties, the other approach considers objects satisfying a particular commutation relation.

\subsection{Some general observations}

Let us start with some general observations. Wyss [133] considered in 1980 the situation where $\mathfrak{B}$ is a $*$-algebra with unit 1 . For elements $A, B, X \in \mathfrak{B}$ one lets

$$
A(X)=A X, \quad d_{B}(X)=[B, X]=B X-X B,
$$

i.e., $A$ acts by left multiplication and $d_{B}$ is a derivation. Both are elements in $\mathcal{L}(\mathfrak{B})$, the set of linear transformations from $\mathfrak{B}$ to $\mathfrak{B}$. Any power $\left(A+d_{B}\right)^{n}, n \in \mathbb{N}$, is also an element in $\mathcal{L}(\mathfrak{B})$ and one has the following binomial formula (which can be shown by induction).

Theorem 5.1. [133, Corollary 1] For $n \in \mathbb{N}$, one has

$$
(A+B)^{n}=\sum_{k=0}^{n}\left(\begin{array}{l}
n \\
k
\end{array}\right)\left\{\left[A+d_{B}\right]^{k} 1\right\} B^{n-k} .
$$

Dividing by $n$ ! and summing over $n$, one obtains $e^{A+B}=\left\{e^{A+d_{B}} 1\right\} e^{B}[133$, Corollary 2]. Recently, Wyss [134] returned to the subject and derived some further expansions. Following [134], we write $\left[A+d_{B}\right]^{n} 1=$ $A^{n}+D_{n}(B, A)$. For a commutative algebra all $D_{n}(B, A)$ are identically zero, so the $D_{n}(B, A)$ were called the essential non-commutative part. It satisfies the recurrence relation $D_{n+1}(B, A)=d_{B} A^{n}+\left(A+d_{B}\right) D_{n}(B, A)$ 
with $D_{0}(B, A)=0$, see [134, Eq. (11)]. If $M_{n}(A, B)=\sum_{k=0}^{n}\left(\begin{array}{c}n \\ k\end{array}\right) A^{k} B^{n-k}$, then we can write (58) equivalently as $[134$, Corollary 1$]$

$$
(A+B)^{n}=M_{n}(A, B)+\sum_{k=0}^{n}\left(\begin{array}{l}
n \\
k
\end{array}\right) D_{k}(B, A) B^{n-k} .
$$

As an example, [134] considered the case where $d_{B}(A)=h A^{2}$ (note that this means explicitly $B A-A B=h A^{2}$, i.e., the commutation relation of the Jordan plane, see Example 1.4). By introducing $\gamma_{k}(h)=\prod_{j=0}^{k-1}(1+j h)$ (note $\gamma_{k}(h)=(1 \mid-h)_{k}$ from Example 1.4), Wyss observed $d_{B} A^{k}=k h A^{k+1}$ and $D_{k}(B, A)=\left\{\gamma_{k}(h)-1\right\} A^{k}$. Using this in (59) yields $(A+B)^{n}=\sum_{k=0}^{n}\left(\begin{array}{l}n \\ k\end{array}\right) \gamma_{k}(h) A^{k} B^{n-k}[134$, Eq. (20)], thereby recovering Benaoum's result (10). In the same paper, one can also find an expansion $(A+B)^{n}=M_{n}(A, B)+\sum_{k=0}^{n-2}(A+B)^{k} d_{B} M_{n-1-k}(A, B)$ [134, Theorem 2], which we will not discuss further. These considerations were continued by Hosseini and Mohammadzadeh Karizaki [68]. Recall that the inner derivation $d_{B}$ acts by $d_{B}(A)=B A-A B$. Given two elements $B_{1}$ and $B_{2}$, a generalized inner derivation $\delta_{B_{1}, B_{2}}$ may be introduced by its action $\delta_{B_{1}, B_{2}}(A)=$ $B_{1} A-A B_{2}$. By induction, one finds $\delta_{B_{1}, B_{2}}^{n}(A)=\sum_{k=0}^{n}(-1)^{k}\left(\begin{array}{l}n \\ k\end{array}\right) B_{1}^{n-k} A B_{2}^{k}[68$, Lemma 1]. This is the main ingredient for the following result.

Theorem 5.2. [68, Theorem 1] For $n \in \mathbb{N}$, one has

$$
A^{n}=\sum_{k=0}^{n}\left(\begin{array}{l}
n \\
k
\end{array}\right) \delta_{A, B}^{n-k}(1) B^{k}
$$

Switching $B$ to $C$ and $A$ to $A+B$, one obtains the binomial formula $(A+B)^{n}=\sum_{k=0}^{n}\left(\begin{array}{l}n \\ k\end{array}\right) \delta_{A+B, C}^{n-k}(1) C^{k}$ for any $A, B, C \in \mathfrak{B}[68$, Corollary 1]. The authors also derived a binomial formula for non-unital algebras (by embedding it into a unital algebra) considered also the case of negative powers.

Before turning to some concrete new results, we describe a nice variant of the binomial formula due to Sills [125]. Recall from Section 2.4 the notations for partitions $\lambda=\left(\lambda_{1}, \lambda_{2}, \ldots, \lambda_{k}\right)$. For a partition $\lambda$, the Young diagram $Y_{\lambda}$ of shape $\lambda$ is a left-justified diagram of $|\lambda|$ boxes with $\lambda_{i}$ black boxes in the $i$-th row. We denote the set of all Young diagrams that are contained in a $k \times(n-k)$ box by $\mathcal{T}_{k, n-k}$, for $1 \leq k \leq n$, with $\mathcal{T}_{0, n}=\left\{n^{0}\right\}$. Equivalently, $\mathcal{T}_{k, n-k}$ can be considered as the set $\mathcal{P}_{k, n-k}$ of partitions $\lambda$ having at most $k$ parts with length at most $n-k$. Note that $\mathcal{P}_{k, n-k}$ contains $\left(\begin{array}{l}n \\ k\end{array}\right)$ elements. If we let $\mathcal{P}_{n}=\cup_{k=0}^{n} \mathcal{P}_{k, n-k}$, then we have the following result.

Theorem 5.3. [99, Theorem 3.2] There exists a bijection between the set $\mathcal{P}_{k, n-k}$ and the binary words over the alphabet $\{U, V\}$ of length $n$ with $k$ occurrences of $V$ and $n-k$ occurrences of $U$.

The main idea is the following. Let $\pi$ be any word over the alphabet $\{U, V\}$ of length $n$ with $k$ occurrences of the letter $V$ in $\pi$ (hence, the letter $U$ occurs $n-k$ times in $\pi$ ). Define the numbers $\lambda_{j}$, for $1 \leq j \leq k$, by (note that in [99] the indices of the partitions are given by $\left.\left(\lambda_{k}, \lambda_{k-1}, \ldots, \lambda_{1}\right) !\right)$

$$
\pi=U^{n-k-\lambda_{1}} V U^{\lambda_{1}-\lambda_{2}} \cdots V U^{\lambda_{k-1}-\lambda_{k}} V U^{\lambda_{k}} .
$$

Then $\lambda=\left(\lambda_{1}, \ldots, \lambda_{k}\right) \in \mathcal{P}_{k, n-k}$. On the other hand, for $\lambda \in \mathcal{P}_{k, n-k}$ we define a word $\pi_{\lambda}$ by (61). Thus, $\pi_{\lambda}$ is a binary word over the alphabet $\{U, V\}$ of length $n$ with exactly $k$ letters of $V$ and exactly $n-k$ letters of $U$. Thus, as mentioned in [99], we can write

$$
(U+V)^{n}=\sum_{k=0}^{n} \sum_{\lambda \in \mathcal{P}_{k, n-k}} U^{n-k-\lambda_{1}} V U^{\lambda_{1}-\lambda_{2}} \cdots V U^{\lambda_{k-1}-\lambda_{k}} V U^{\lambda_{k}} .
$$

Each $U^{n-k-\lambda_{1}} V U^{\lambda_{1}-\lambda_{2}} \ldots V U^{\lambda_{k-1}-\lambda_{k}} V U^{\lambda_{k}}$ has $k$ letters of $V$ and $n-k$ letters of $U$. The idea of Sills [125] is to define for each $\lambda \in \mathcal{P}_{k, n-k}$ an operator $Q^{\lambda}$ such that $Q^{\lambda}\left(U^{n-k} V^{k}\right)=U^{n-k-\lambda_{1}} V U^{\lambda_{1}-\lambda_{2}} \cdots V U^{\lambda_{k-1}-\lambda_{k}} V U^{\lambda_{k}}$.

Theorem 5.4. [125, Theorem 1] For $n \in \mathbb{N}$, one has the non-commutative binomial theorem

$$
(U+V)^{n}=\sum_{k=0}^{n} \sum_{\lambda \in \mathcal{P}_{k, n-k}} Q^{\lambda}\left(U^{n-k} V^{k}\right) .
$$


For $\lambda=\left(\lambda_{1}, \ldots, \lambda_{k}\right) \in \mathcal{P}_{k, n-k}$ the operator $Q^{\lambda}$ is defined as a product of transpositions

$$
Q^{\lambda}=\left(n, n-\lambda_{k}\right) \cdots\left(n-k+j, n-k+j-\lambda_{j}\right) \cdots\left(n-k+1, n-k+1-\lambda_{1}\right) .
$$

The transposition $(i, j)$ applied to $U^{n-k} V^{k}$ means that the $i$-th and $j$-th factor in $U^{n-k} V^{k}$ are swapped. The order of factors is not commutative, and the notation above means that $\left(n-k+1, n-k+1-\lambda_{1}\right)$ is applied first, then $\left(n-k+2, n-k+2-\lambda_{2}\right)$, and so on. As an example, we choose the one considered in [125]. Let $n=8$ and $k=3$, so we consider $U^{5} V^{3}$. For $\lambda=(3,1,0)$ we should get $Q^{\lambda}\left(U^{5} V^{3}\right)=U^{2} V U^{2} V U V$, according to above. The definition of $Q^{\lambda}$ gives

$$
\begin{aligned}
Q^{(3,1,0)}\left(U^{5} V^{3}\right) & =(8,8)(7,6)(6,3)(U U U U U V V V)=(8,8)(7,6)(U U V U U U V V)=(8,8)(U U V U U V U V) \\
& =U U V U U V U V=U^{2} V U^{2} V U V,
\end{aligned}
$$

as it should be. If $U$ and $V$ commute, then $Q^{\lambda}\left(U^{n-k} V^{k}\right)=U^{n-k} V^{k}$ and since $\left|\mathcal{P}_{k, n-k}\right|=\left(\begin{array}{l}n \\ k\end{array}\right)$, one recovers the conventional binomial theorem. For $U V=q V U$, one has $U^{n-k-\lambda_{1}} V U^{\lambda_{1}-\lambda_{2}} \cdots V U^{\lambda_{k-1}-\lambda_{k}} V U^{\lambda_{k}}=$ $q^{k(n-k)-|\lambda|} V^{k} U^{n-k}$, so (62) gives immediately

$$
(U+V)^{n}=\sum_{k=0}^{n}\left(\sum_{\lambda \in \mathcal{P}_{k, n-k}} q^{k(n-k)-|\lambda|}\right) V^{k} U^{n-k} .
$$

Using that $\sum_{\lambda \in \mathcal{P}_{k, n-k}} q^{k(n-k)-|\lambda|}=\sum_{\lambda \in \mathcal{P}_{k, n-k}} q^{|\lambda|}$ and the well-known relation $\sum_{\lambda \in \mathcal{P}_{k, n-k}} q^{|\lambda|}=\left(\begin{array}{l}n \\ k\end{array}\right)_{q}$, one recovers (4), the $q$-binomial theorem.

\section{$5.2 \quad$ A few recent concrete cases}

In the preceding section, we discussed the binomial theorem in several variants for non-commuting variables where no commutation relation was assumed. So, to obtain more concrete results, we have to assume some commutation relation for the variables $U$ and $V$ to be able to simplify the right-hand side further. In this section, we point out a few recent developments in this direction.

\subsubsection{The quantum plane revisited}

We start by recalling (4), where the variables $U$ and $V$ are assumed to $q$-commute. A new interpretation for the $q$-binomial coefficients $\left(\begin{array}{l}n \\ k\end{array}\right)_{q}$ was given recently by Przytycki [117] who considered the tree $T_{b, a}$ of two long branches of length $b$ and $a$, respectively, originating from a common root. The conventional binomial coefficient $\left(\begin{array}{c}a+b \\ a\end{array}\right)$ then counts the number of how many different ways there are to "pluck" the tree $T_{b, a}$ one leaf at a time. Introducing a weight $q^{a}$ when plucking a leaf from the " $a$-branch", one gets instead the plucking polynomial $Q\left(T_{b, a}\right)$, which is given by $\left(\begin{array}{c}a+b \\ a, b\end{array}\right)_{q}=\frac{[a+b]_{q} !}{[a]_{q} ![b]_{q} !}$. In a similar fashion, $q$-multinomial coefficients for $k$ variables were interpreted as plucking polynomials for a tree with $k$ long branches. Finally, this was generalized by introducing for plane rooted trees a plucking polynomial [117, Definition 2.1] in a recursive fashion. Several properties of the plucking polynomial were shown in [117], and it as been studied further in subsequent papers.

\subsubsection{The monomial generalization of the quantum plane}

Recall that variables $U$ and $V$ satisfying the commutation relation $U V=q V^{s} U$ were considered in Section 4.6. A binomial formula for these variables was discussed in [99]. To state this result, we introduce another convenient notation for partitions in the following way. We write each partition $\lambda \in \mathcal{P}_{k, n-k}$ equivalently as $\nu(\lambda)=$ $\nu_{d}^{\ell_{d}} \nu_{d-1}^{\ell_{d-1}} \cdots \nu_{1}^{\ell_{1}}, \nu_{0}=0$ and $\nu_{d+1}=n-k$, where $\nu_{d+1}=n-k \geq \nu_{d}>\nu_{d-1}>\cdots>\nu_{1} \geq \nu_{0}=0$ and $\ell_{1}, \ldots, \ell_{d} \geq 1$. Thus, the $\nu_{j}$ describe the lengths of the rows of the associated Young diagram (starting from the top), and $\ell_{j}$ denotes how often the row of this length occurs. For example, if $\lambda=44222111 \in \mathcal{P}_{8,4}$, then $\nu(\lambda)=4^{2} 3^{0} 2^{3} 1^{3} 0^{0}=4^{2} 2^{3} 1^{3}$. We also introduce the notation $\mathcal{R}_{k, n-k}=\left\{\nu(\lambda) \mid \lambda \in \mathcal{P}_{k, n-k}\right\}$. For instance, $\mathcal{R}_{0,4}=\left\{4^{0}\right\}, \mathcal{R}_{1,3}=\{0,1,2,3\}, \mathcal{R}_{2,2}=\left\{0^{2}, 10,20,1^{2}, 21,2^{2}\right\}, \mathcal{R}_{3,1}=\left\{0^{3}, 10^{2}, 1^{2} 0,1^{3}\right\}$ and $\mathcal{R}_{4,0}=\left\{0^{4}\right\}$. 
Theorem 5.5. [99, Theorem 3.4] Let $U$ and $V$ satisfy $U V=q V^{s} U$ with $s \in \mathbb{N}$. Then one has, for all $n \in \mathbb{N}$, the following binomial formula,

$$
(U+V)^{n}=\sum_{k=0}^{n}\left\{\sum_{\nu \in \mathcal{R}_{k, n-k}} q^{\sum_{i=1}^{d} \sum_{j=i}^{d} \ell_{i}\left[\nu_{j+1}-\nu_{j}\right]_{s} s^{\nu_{j}-\nu_{i}}} V^{\sum_{i=1}^{d} \ell_{i} s^{n-k-\nu_{i}}}\right\} U^{n-k} .
$$

Problem 5.1. It would be interesting to find a combinatorial interpretation for the coefficients appearing in (63). See also Problem 4.2 for a related problem.

\subsubsection{Weight-dependent commutation relations}

In Section 5.2.1, we mentioned an interpretation for the $q$-binomial coefficients (appearing in the binomial formula for the variables $U$ and $V$ satisfying $U V=q V U$ ) as plucking polynomial for simple rooted trees. It is also possible to interpret it in terms of weighted lattice paths, see Schlosser and Yoo $[122,123]$. The algebraic expression " $V U$ " would refer to a path going one step east, then one step north, while " $U V$ " would refer to a path going one step north, then one step east (in this section, the steps of a path will be read from left to right). Keeping track of the area under the paths and assigning the weight of a path $P$ to be $q^{a}$ when the area under $P$ is $a$, we see that the weight of the path $U V$ is $q$, while that of $V U$ is 1 . Thus, the commutation relation $U V=q V U$ describes the change of weights when the two steps are interchanged.

Schlosser [121] has generalized this as follows. For a doubly-indexed sequence of indeterminates $(w(s, t))_{s, t \in \mathbb{N}}$ let $\mathbb{C}_{w}[U, V]$ be the associative unital algebra over $\mathbb{C}$ generated by $U, V$ and the $w(s, t)$, with $s, t \in \mathbb{N}$ satisfying the following relations,

$$
U V=w(1,1) V U, \quad V w(s, t)=w(s+1, t) V, \quad U w(s, t)=w(s, t+1) U .
$$

The $w(s, t)$ (and products of them) are referred to as weights. If $w(s, t)=q$ for all $s, t \in \mathbb{N}$, then one recovers the quantum plane, see Example 1.2. Let us introduce $W(s, t)=w(s, 1) w(s, 2) \cdots w(s, t)$. The weight-dependent binomial coefficients are defined by $\left[\begin{array}{l}0 \\ 0\end{array}\right]_{w}=1$ and $\left[\begin{array}{l}n \\ k\end{array}\right]_{w}=0$, for $n \in \mathbb{N}_{0}$ and $k>n$, as well as the recurrence

$$
\left[\begin{array}{c}
n+1 \\
k
\end{array}\right]_{w}=\left[\begin{array}{l}
n \\
k
\end{array}\right]_{w}+\left[\begin{array}{c}
n \\
k-1
\end{array}\right]_{w} W(k, n+1-k)
$$

for $n, k \in \mathbb{N}_{0}$. By an induction, the following weight-dependent binomial theorem is shown.

Theorem 5.6. [121, Theorem 1] For $n \in \mathbb{N}$, one has the following identity in $\mathbb{C}_{w}[U, V]$,

$$
(V+U)^{n}=\sum_{k=0}^{n}\left[\begin{array}{l}
n \\
k
\end{array}\right]_{w} V^{k} U^{n-k}
$$

The first relation of (64) can be generalized by induction to $U^{k} V^{l}=\left(\prod_{i=1}^{l} W(i, k)\right) V^{l} U^{k}[121$, Lemma 1]. Let us turn to the interpretation in terms of weighted lattice paths. For this, assign to each horizontal step $(s-1, t) \rightarrow(s, t)$ the weight $W(s, t)$, and to each vertical step $(s, t-1) \rightarrow(s, t)$ the weight 1 . The weight of a path $P, \omega(P)$, is defined to be the product of the weights of all steps. For example, in Figure 8 a path $P_{0}$ from $(0,0)$ to $(7,4)$ is shown. It has weight

$$
\omega\left(P_{0}\right)=1 \cdot 1 \cdot 1 \cdot W(2,2) \cdot W(3,2) \cdot W(4,2) \cdot 1 \cdot W(5,3) \cdot 1 \cdot W(6,4) \cdot W(7,4),
$$

where we used $W(1,0)=1$.

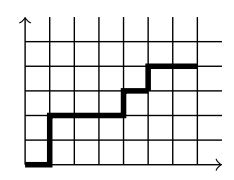

Figure 8: The lattice path $P_{0}$ from $(0,0)$ to $(7,4)$. 
Given $A, \Omega \in \mathbb{N}_{0}^{2}$, let $\mathcal{P}(A \rightarrow \Omega)$ be the set of all paths from $A$ to $\Omega$. Further, let $\omega(\mathcal{P}(A \rightarrow \Omega))=$ $\sum_{P \in \mathcal{P}(A \rightarrow \Omega)} \omega(P)$. Then one has [121, Eq. (2.5)]

$$
\left[\begin{array}{l}
n \\
k
\end{array}\right]_{w}=\omega(\mathcal{P}((0,0) \rightarrow(k, n-k)))
$$

As discussed in [121], the identification of words in $\mathbb{C}_{w}[U, V]$ and lattice paths in $\mathbb{Z}^{2}$ works locally (step by step) as follows: $U$ (resp., $V$ ) corresponds to a vertical (resp., horizontal) step. Under this correspondence, " $V U$ " means that a horizontal step is followed by a vertical step, while " $U V$ " means that a vertical step is followed by a horizontal step. The commutation relation (64) take into account the changes of the weights when consecutive horizontal and vertical steps are exchanged. For example, the path $P_{0}$ considered above corresponds to the algebraic expression (here the relations (64) have to be used repeatedly)

$$
V U U V V V U V U V V=W(2,2) W(3,2) W(4,2) W(5,3) W(6,4) W(7,4) V^{7} U^{4}=\omega\left(P_{0}\right) V^{7} U^{4} .
$$

Remark 5.1. We can make contact with (62) since there exists a bijection between the words of length $n$ with exactly $k$ appearances of $V$ and a lattice path of length $n$ with $k$ horizontal steps. If we assume that the commutation relations (64) hold true, then we can write

$$
U^{n-k-\lambda_{1}} V U^{\lambda_{1}-\lambda_{2}} \cdots V U^{\lambda_{k-1}-\lambda_{k}} V U^{\lambda_{k}}=\left(\prod_{j=1}^{k} W\left(j, n-k-\lambda_{j}\right)\right) V^{k} U^{n-k},
$$

where we have commuted successively the powers of $U$ to the right, using $U^{m} V=W(1, m) V U^{m}$ as well as $V^{r} W(s, t)=W(s+r, t) V^{r}$ following from (64). For example, the expression $V U U V V V U V U V V$ corresponding to the path $P_{0}$ from Figure 8 is associated to the partition $\lambda=(4,2,2,2,1,0,0,0)$, and the factor in front of the normal ordered term in $(69)$ is precisely the weight $\omega\left(P_{0}\right)$ of the path $P_{0}$ given in (67).

Schlosser [121] considered several specializations of (64), in particular elliptic commuting variables, and derived the corresponding binomial theorems for these cases. This line of study was continued by Schlosser and Yoo [122,123], where an elliptic analogue of rook numbers was defined, and with their help elliptic Stirling numbers (recall that the Stirling numbers of the second kind have a nice interpretation in terms of rook numbers of the staircase board $\left.J_{n}, S(n, k)=r_{n-k}\left(J_{n}\right)\right)$.

Schlosser [121, Appendix A] generalized the above considerations by introducing also a weight for vertical steps. To be precise, the step from $(s, t-1) \rightarrow(s, t)$ has weight $v(s, t)$ (instead of the constant value 1 above). For example, the path $P_{0}$ from Figure 8 has now weight

$$
\tilde{\omega}\left(P_{0}\right)=v(1,1) \cdot v(1,2) \cdot W(2,2) \cdot W(3,2) \cdot W(4,2) \cdot v(3,4) \cdot W(5,3) \cdot v(5,4) \cdot W(6,4) \cdot W(7,4),
$$

instead of $(67)$. In analogy to $\mathbb{C}_{w}[U, V]$, one can introduce for the two doubly-indexed sequences $(v(s, t))_{s, t \in \mathbb{N}}$ and $(w(s, t))_{s, t \in \mathbb{N}}$ the associative unital algebra $\mathbb{C}_{v, w}[U, V]$ generated by the $U, V$ and the $v(s, t)$ and $w(s, t)$, $s, t \in \mathbb{N}$, satisfying (64) and in addition $U v(s, t)=v(s, t+1) U$ and $V v(s, t)=v(s+1, t) V$. The corresponding double weight-dependent binomial coefficients are defined in analogy to above and satisfy the recurrence relation

$$
\left[\begin{array}{c}
n+1 \\
k
\end{array}\right]_{v, w}=\left[\begin{array}{l}
n \\
k
\end{array}\right]_{v, w} v(k, n+1-k)+\left[\begin{array}{c}
n \\
k-1
\end{array}\right]_{v, w} W(k, n+1-k) .
$$

They again have a combinatorial meaning in terms of weighted lattice paths, $\left[\begin{array}{l}n \\ k\end{array}\right]_{v, w}=\tilde{\omega}(\mathcal{P}((0,0) \rightarrow(k, n-k)))$, generalizing (68). Generalizing Theorem 5.6, one has the following double weight-dependent binomial theorem.

Theorem 5.7. [121, Theorem 3] For $n \in \mathbb{N}$, one has the following identity in $\mathbb{C}_{v, w}[U, V]$,

$$
(V+v(0,1) U)^{n}=\sum_{k=0}^{n}\left[\begin{array}{l}
n \\
k
\end{array}\right]_{v, w} V^{k} U^{n-k} .
$$

As mentioned in [121], by choosing $w(s, t)=1$, for $s, t \in \mathbb{N}$, and $v(s, t)=[s]_{q}$, for $s, t \in \mathbb{N}$, (71) shows that in that case $\left[\begin{array}{l}n \\ k\end{array}\right]_{v, w}=S_{q}(n, k)$, the $q$-deformed Stirling numbers of the second kind. 
Recall that (64) constitutes a generalization of the commutation relation $U V=q V U$ of the quantum plane. Schlosser and Yoo [123] considered the slighty modified system

$$
U V=w(1,1) V U+1, \quad V w(s, t)=w(s+1, t) V, \quad U w(s, t)=w(s, t+1) U,
$$

which constitutes a generalizaton of the $q$-deformed Weyl algebra where $U V=q V U+1$, see Example 1.3. They showed [123, Theorem 4.1] that one can write a word $\alpha$ consisting of $n$ letters $U$ and $m$ letters $V$ in the normal ordered form $\alpha=\sum_{k} r_{k}\left(w, B_{\alpha}\right) V^{m-k} U^{n-k}$ where the normal ordering coefficients $r_{k}\left(w, B_{\alpha}\right)$ can be interpreted in terms of weighted rook numbers of the board $B_{\alpha}$ associated to $\alpha$. In a similar fashion, they considered the system where the first relation in (73) is modified to $U V=w(1,1) V U+U$ and showed [123, Theorem 4.4] the analogous normal ordering result $\alpha=\sum_{k} f_{k}\left(w, B_{\alpha}\right) V^{m-k} U^{n}$ where the normal ordering coefficients $f_{k}\left(w, B_{\alpha}\right)$ can be interpreted in terms of weighted file numbers of the board $B_{\alpha}$ associated to $\alpha$. (Recall that a file placement of $k$ rooks on a board $B$ is a placement of $k$ rooks on $B$ such that no two rooks lie in the same column. The $k$-th file number $f_{k}(B)$ of a board $B$ is the number of possible file placements of $k$ rooks.)

Problem 5.2. a) Recall from Section 4.3 that the normal ordering coefficients for a word in letters $U$ and $V$ satisfying $U V=q V U+V^{s}, s \in \mathbb{N}$, can be interpreted in terms of s-rook numbers. The above described cases correspond to $s=0$ and $s=1$. Consider the system

$$
U V=w(1,1) V U+U^{s}, \quad V w(s, t)=w(s+1, t) V, \quad U w(s, t)=w(s, t+1) U,
$$

Give a combinatorial interpretation for the normal ordering coefficients of words in $U$ and $V$. The case $s=2$ constitutes a generalization of the Jordan plane (see Example 1.4) which has many nice properties. What about the generalization of the binomial formula? At least in the cases $s=0,1,2$ one should have an interesting interpretation for the corresponding coefficients. Finally, consider in these cases the double weighted situation by introducing an additional weight sequence $v(s, t)$ (similar to above). b) Consider the system where the first relation in (73) is modified to $U V=w(1,1) V U+U+V$. Note that this would constitute a generalization of the $q$-deformed excedance algebra, see Section 4.5.

\subsubsection{Curious binomial-type identities}

Roughly 30 years ago, Kuchment and Lvin $[84,85]$ worked on the theory of medical imaging and found a remarkable identity. In [86], this identity was discussed in more detail and more context was given. We follow the presentation in [86] and let $A$ be a unital commutative algebra (over a field $\mathbb{K}$ ) with cancellation property and derivation $D$ (i.e., $D(v w)=D(v) w+v(D(w))$.

Theorem 5.8. [84, Lemma] Any solution $u$ of the first-order equation $D u=\lambda u, \lambda \in \mathbb{K}$, satisfies for any $n \in \mathbb{N}$ the following identity:

$$
\sum_{k=0}^{n}\left(\begin{array}{l}
n \\
k
\end{array}\right)(D-u) \circ(D-u+\lambda) \circ \ldots \circ(D-u+(k-1) \lambda) u^{n-k}=0 .
$$

Any solution $u$ of the second-order equation $D^{2} u=\lambda^{2} u, \lambda \in \mathbb{K}$, satisfies for any odd $n \in \mathbb{N}$ identity (74).

In the above identity, the expressions like $(D-u-j \lambda)$ are considered as operators on the algebra $A$, acting to the elements on the right. The symbol o means composition of the operators from right to left. The term for $k=0$ is just $u^{n}$. For example, using $u^{0}=1,(74)$ reduces for $n=1$ to the trivial identity $u+(D-u) 1=0$. For $n=2$, one obtains for the left-hand side $u^{2}+2(D-u) u+(D-u) \circ(D-u+\lambda) 1$ which reduces to $D u-\lambda u$. This vanishes by the assumption on $u$.

For the proof of Theorem 5.8 we refer to [86]. There one can also find discussions on pitfalls and similarities to the commutative situation. Note that for $A=C^{\infty}(\mathbb{R})$ over $\mathbb{K}=\mathbb{C}$ with $\lambda=i$ (with $i^{2}=-1$ ) the derivation $D$ is just the usual derivative, and $u(x)=\sin (x)$ satisfies $D^{2} u=-u$. Thus, (74) gives an identity for $\sin (x)$ (for $n$ odd) and was the one used in $[84,85]$. Choosing $\lambda=1$ and $u=e^{x},(74)$ gives the identity

$$
\sum_{k=0}^{n}\left(\begin{array}{l}
n \\
k
\end{array}\right)\left(\frac{d}{d x}-e^{x}\right) \circ\left(\frac{d}{d x}-e^{x}+1\right) \circ \ldots \circ\left(\frac{d}{d x}-e^{x}+(k-1)\right) e^{(n-k) x}=0
$$


observed in [86]. Let us mention the following observation [86]: If we let $P_{n}(D, X)=\sum_{k=0}^{n}\left(\begin{array}{c}n \\ k\end{array}\right) D^{k} X^{n-k}$, then one has the recurrence relation $P_{n+2}(D, X)=P_{n+1}(D, X)(D+X)+(n+1) P_{n}(D, X)(X$ denotes the operator of multiplication with $x$ ).

More recently, Kuchment and Lviv [87] returned to identity (74) and generalized it in the following situation. Let $D$ and $u$ be elements of an associative algebra $A$ over a field $\mathbb{K}$ with unit $I$. The left-hand side of (74) will be denoted by $B(n, \lambda, u, D)$ (where $\lambda \in \mathbb{K}$ and $j \lambda$ means $j \lambda I$ ). Depending on the assumption on the commutator $[D, u]=D u-u D$, analogous results to Theorem 5.8 were shown in [87]. For example, if $[D, u]=\lambda u$ (the shift algebra $)$, then $B(n, \lambda, u, D)$ does not depend on $u, B(n, \lambda, u, D)=B(n, \lambda, 0, D)=D(D+\lambda) \cdots(D+(n-1) \lambda)$. As a concrete example, one can consider $A$ to be the algebra of differential operators on $C^{\infty}(\mathbb{R})$ with $D=\frac{d}{d x}$ and $u=e^{\lambda x}$ (as multiplication operator). One has $\left[D, e^{\lambda x}\right]=\lambda e^{\lambda x}$ and since $e^{-j \lambda x}$ belongs to the kernel of $(D+j \lambda)$ one has $B\left(n, \lambda, e^{\lambda x}, D\right) e^{-j \lambda x}=0$ (for all $n>0$ and $0 \leq j \leq n-1$ ) as analogue of (74). In a similar fashion, the second-order equation from Theorem 5.8 gets replaced in the present situation by a condition on the second-order commutators. For example, one has [87, Theorem 1.5]: If $[D,[D, u]]=\lambda^{2} u$ and $[u,[D, u]]=0$, then $\left.B(n, \lambda, u, D)\right|_{A_{0}}=0$ for all odd $n \in \mathbb{N}$ (where $A_{0}=\{V \in A \mid D V=0\}$ ). Several further results can be found in [87].

Problem 5.3. It would be interesting to find a combinatorial proof of Theorem 5.8. Also, as Kuchment and Lvin mentioned themselves [86], it would be natural to consider what happens to solutions of higher-order differential equations, $D^{m} u=\lambda^{m} u$. Can one find analogous identities? As it seems, a natural analogue of Theorem 5.8 does not exist, see the remarks in [86,87].

\section{Miscellaneous research related to normal ordering}

In this section, we point out some recent research where the ordering of non-commuting obects plays a role for the investigation of the main objective.

- Briand et al. [25] studied the commutation relations and normal ordering for certain families of operators on symmetric functions. These operators can be naturally defined by the operations of multiplication, Kronecker product, and their adjoints. In total, four families of operators $U_{\lambda}, D_{\lambda}, K_{\lambda}$ and $\bar{K}_{\lambda}$ associated to a partition $\lambda$ were studied and the commutation relations were derived [25, Theorem 1.1]. Also, the question "Given a word involving these operators, how can we put it in a normal form?" was discussed.

- Méndez and Ramírez [104] considered the $r$-Whitney numbers of the first and second kind (see Section 2.2) and gave new combinatorial interpretations for many properties by using combinatorial differential operators in the form discussed by Méndez [103] (see also Labelle and Lamathe [88]). Among the many relations shown - or recovered - by their new approach they derived [104, Theorem 5] the Spivey-like relation (33) (for $q=1$ ).

- Škoda [126] considered symmetric ordering in the $n$-th Weyl algebra $A_{n}$. (Recall that symmetric ordering a monomial in the generators means to sum over all possible arrangements in the generators, see [98] and the references therein).

- Cantuba [30] began the study of the Lie polynomials of the $q$-deformed Weyl algebra when $q$ is not a root of unity, see Example 1.3. If we let $U, V$ be the generators satisfying $U V-q V U=I$, then the set of Lie polynomials is the Lie algebra $\mathcal{L}(q)$ generated by $U$ and $V$. For $q \neq 1$, the relation $U V-q V U=I$ cannot be expressed in terms of Lie algebra operations, yet it has consequences for $\mathcal{L}(q)$. Ordering results were needed in the study of $\mathcal{L}(q)$, and the limit $q=0$ was also considered in [30]. Cantuba and Silvestrov [33] continued this study by considering the Weyl algebra when $q$ is a root of unity. Cantuba [31] considered the same problem for the algebra defined by a linearly twisted commutation relation, $U V=\sigma(V U)$ (see Section 4.1), and, together with Merciales [32] for the extension $\mathcal{R}(q)$ of the $q$-deformed Weyl algebra described in Section 4.2. A survey of these results was given by Cantuba and Silvestrov [34].

- Josuat-Vergès [73] considered the derivative polynomials of tangens and secans introduced by Knuth and Buckholtz [83] by $\frac{d^{n}}{d x^{n}} \tan (x)=P_{n}(\tan (x))$ and $\frac{d^{n}}{d x^{n}} \sec (x)=Q_{n}(\tan (x)) \sec (x)$. By letting $\frac{d^{n}}{d x^{n}} \sec ^{a}(x)=$ 
$Q_{n}^{(a)}(\tan (x)) \sec ^{a}(x)$ for $a \in \mathbb{N}$, one generalizes $Q_{n} \equiv Q_{n}^{(1)}$. Let $R_{n} \equiv Q_{n}^{(2)}$. It follows that $P_{n+1}(t)=$ $\left(1+t^{2}\right) R_{n}(t)$. For these two familes of polynomials $Q_{n}, R_{n}$ certain $q$-generalizations were introduced by letting [73, Definition 2.2] (here $1=t^{0}$ )

$$
Q_{n}(t, q)=\left(D_{q}+U D_{q} U\right)^{n} 1, \quad R_{n}(t, q)=\left(D_{q}+D_{q} U U\right)^{n} 1,
$$

where $D_{q} t^{n}=[n]_{q} t^{n-1}$ is the Jackson derivative (8) and $U t^{n}=t^{n+1}$ is the multiplication operator satisfying $D_{q} U=q U D_{q}+1$, the commutation relation of the $q$-deformed Weyl algebra, see Example 1.3. One has $Q_{n}(t, 1)=Q_{n}(t)$ and $R_{n}(t, 1)=R_{n}(t)$ [73, Proposition 2.4]. As observed in [73], using the normal ordered form $\left(D_{q}+U D_{q} U\right)^{n}=\sum_{i, j \geq 0} c_{i, j} U^{i} D_{q}^{j}$ (where the normal ordering coefficients $c_{i, j}$ have to be determined), one finds $Q_{n}(t, q)=\sum_{i, j \geq 0} c_{i, j} U^{i} D_{q}^{j} 1=\sum_{i \geq 0} c_{i, 0} t^{i}$ (since $D_{q}^{j} 1=0$ for $j \geq 1$ ). Several combinatorial interpretations for $Q_{n}(t, q)$ and $R_{n}(t, q)$ (and, in particular, for $q=1$ ) were discussed in [73]. One of these follows by using a general result on normal ordering [22, Theorem 1], see [73, Section 4.2]. Let us mention that the tangent numbers (A000182 in [127]) and secant numbers (or also Euler numbers, A000364 in [127]) are the coefficients in the formal power series for tan and sec and that [83] aimed at an efficient method for computing them. They can be determined by evaluating the polynomials $P_{n}(t), Q_{n}(t), R_{n}(t)$ at $t=1$, see [73]. A completely different approach for computing these numbers using 3 non-commuting operators $R, L, S$ was given by Hodges and Sukumar [67,129]. These operators satisfy the commutation relations

$$
R L-L R=S, \quad R S-S R=2 R, \quad S L-L S=2 L,
$$

and can be considered as generators of the Lie algebra $s u(1,1)$. The tangent and secant numbers can then determined from the powers $(R+L)^{m}$, see the discussion in [67,129]. See also Hetyei [66] for further ramifications, in particular the connection to Viennot's combinatorial theory of orthogonal polynomials.

Remark 6.1. According to Section 4.7, we can associate to the algebra with generators $R, L, S$ satisfying (75) its commutation graph $K_{3}$, the triangle graph. Note that from all commutation graphs mentioned in this paper, this is the only one containing a nontrivial cycle.

- Behr et al. [12] studied single-species chemical reactions by either combinatorial normal ordering techniques, or, for binary reactions, by means of Sobolev-Jacobi orthogonal polynomials. Note that the singlespecies case corresponds to the Weyl algebra $A_{1}$, while the multi-species case - also considered in [12] corresponds to the $n$-th Weyl algebra $A_{n}$ (see Section 3.1) where the generators $\left\{\hat{a}_{i}, \hat{a}_{i}^{\dagger}\right\}_{1 \leq i \leq n}$ satisfy (52). Using the following convenient multi-index notation,

$$
\underline{m} !=\prod_{i} m_{i} !, \quad\left(\frac{m}{\underline{k}}\right)=\prod_{i}\left(\begin{array}{c}
m_{i} \\
k_{i}
\end{array}\right), \quad \underline{x} \underline{y}=\prod_{i}\left(x_{i}^{y_{i}}\right),
$$

one has in $A_{n}$ the following normal ordering result [12, Eq. (46)],

$$
\left(\underline{\hat{a}}^{\dagger}\right)^{\underline{p}} \underline{\hat{a}}^{\underline{q}}\left(\underline{\hat{a}}^{\dagger}\right)^{\underline{k}} \underline{\hat{a}}^{\underline{l}}=\sum_{\underline{i} \geq \underline{0}}\left(\frac{q}{\underline{i}}\right)\left(\begin{array}{l}
\underline{k} \\
\underline{i}
\end{array}\right) \underline{i} !\left(\underline{\hat{a}}^{\dagger}\right) \underline{p}^{\underline{p}}+\underline{k}-\underline{i} \underline{\hat{a}}^{\underline{q}+\underline{l}-\underline{i}},
$$

extending the case $n=1$ from (46). Furthermore, for real vectors $\underline{\alpha}, \underline{\beta} \in \mathbb{R}^{n}$ and for all Taylor expandable functions $f$ one has the reordering result [12, Proposition 1]

$$
e^{\underline{\alpha} \cdot \underline{\hat{a}}} f\left(\underline{\hat{a}}^{\dagger}, \underline{\hat{a}}\right) e^{\underline{\beta} \cdot \underline{a}^{\dagger}}=e^{\underline{\alpha} \cdot \underline{\beta}} e^{\underline{\beta} \cdot \underline{a}^{\dagger}} f\left(\underline{\hat{a}}^{\dagger}+\underline{\alpha}, \underline{\hat{a}}+\underline{\beta}\right) e^{\underline{\alpha} \cdot \underline{\hat{a}}} .
$$

Problem 6.1. Similar to the case $n=1$ - where (47) is the main input for (46) - the main input for (76) in the case $n=2$ can be written explicitly

$$
\hat{a}_{1}^{m_{1}} \hat{a}_{2}^{m_{2}}\left(\hat{a}_{1}^{\dagger}\right)^{m_{3}}\left(\hat{a}_{2}^{\dagger}\right)^{m_{4}}=\sum_{i_{1} \geq 0} \sum_{i_{2} \geq 0}\left(\begin{array}{c}
m_{1} \\
i_{1}
\end{array}\right)\left(\begin{array}{c}
m_{3} \\
i_{1}
\end{array}\right)\left(\begin{array}{c}
m_{2} \\
i_{2}
\end{array}\right)\left(\begin{array}{c}
m_{4} \\
i_{2}
\end{array}\right) i_{1} ! i_{2} !\left(\hat{a}_{1}^{\dagger}\right)^{m_{3}-i_{1}}\left(\hat{a}_{2}^{\dagger}\right)^{m_{4}-i_{2}} \hat{a}_{1}^{m_{1}-i_{1}} \hat{a}_{2}^{m_{2}-i_{2}},
$$

where we used indices adapted to a result we want to quote. For $n=1$, the coefficient $\left(\begin{array}{c}m_{1} \\ i\end{array}\right)\left(\begin{array}{c}m_{3} \\ i\end{array}\right)$ i is the $i$-th rook number of the full $\left[m_{1}\right] \times\left[m_{3}\right]$ board $B_{m_{1}, m_{3}}$, i.e., $r_{i}\left(B_{m_{1}, m_{3}}\right)=\left(\begin{array}{c}m_{1} \\ i\end{array}\right)\left(\begin{array}{c}m_{3} \\ i\end{array}\right) i$ ! Thus,

$$
\hat{a}_{1}^{m_{1}} \hat{a}_{2}^{m_{2}}\left(\hat{a}_{1}^{\dagger}\right)^{m_{3}}\left(\hat{a}_{2}^{\dagger}\right)^{m_{4}}=\sum_{i_{1} \geq 0} \sum_{i_{2} \geq 0} r_{i_{1}}\left(B_{m_{1}, m_{3}}\right) r_{i_{2}}\left(B_{m_{2}, m_{4}}\right)\left(\hat{a}_{1}^{\dagger}\right)^{m_{3}-i_{1}}\left(\hat{a}_{2}^{\dagger}\right)^{m_{4}-i_{2}} \hat{a}_{1}^{m_{1}-i_{1}} \hat{a}_{2}^{m_{2}-i_{2}},
$$


and it is clear that an analogous factorization holds for arbitrary $n \geq 2$. On the other hand, one may ask for an interpretation in terms of rook numbers of higher dimensional boards, as discussed by Alayont and Krzywonos [6]. For example, if one considers the full $\left[m_{1}\right] \times\left[m_{2}\right] \times \cdots \times\left[m_{2 n}\right]$ "board" $B_{m_{1}, \ldots, m_{2 n}}$ in $2 n$ dimensions, its $i$-th rook number is given by $\left(\begin{array}{c}m_{1} \\ i\end{array}\right)\left(\begin{array}{c}m_{2} \\ i\end{array}\right) \cdots\left(\begin{array}{c}m_{2 n} \\ i\end{array}\right)(i \text { ! })^{2 n-1}$, see [6]. (If a rook is placed in cell $\left(j_{1}, \ldots, j_{2 n}\right)$, then another rook may not be placed in any other cell sharing a coordinate with this cell.) If we compare this with (77), we see that the diagonal coefficients with $i_{2}=i_{1}$ can be described as $\frac{1}{i_{1} !} r_{i_{1}}\left(B_{m_{1}, \ldots, m_{4}}\right)$, but other summands contribute as well. Therefore, it would be interesting to give an interpretation for the normal ordering coefficients of arbitrary words in $A_{n}$ by rook numbers or other combinatorial constructions on $2 n$-dimensional boards, giving an alternative interpretation to the one of digraphs considered in Section 3.1.

- The Sobolev-Jacobi polynomials mentioned in the previous point were studied by Behr et al. [8] using operational - and, in particular, normal ordering - methods. Let us mention the following nice observation [8, Lemma 2]: For any entire function $f$ in the Euler operator $\mathcal{E}=X D$ (see Section 2.1.1) and any $p, q \in \mathbb{N}$ one has

$$
f(\mathcal{E}) X^{p} D^{q}=X^{p} D^{q} f(\mathcal{E}+p-q), \quad X^{p} D^{q} f(\mathcal{E})=f(\mathcal{E}-p+q) X^{p} D^{q}
$$

- Blasiak et al. [20] gave a combinatorial derivation of the Glaisher-Crofton identity,

$$
\exp \left(\alpha \frac{d^{2}}{d x^{2}}\right) \exp \left(-x^{2}\right)=\frac{1}{\sqrt{1+4 \alpha}} \exp \left(-\frac{x^{2}}{1+4 \alpha}\right)
$$

Although not directly related to normal ordering, the authors of [20] mentioned that the combinatorial approach to derivatives can provide insights into different operator identities, e.g., those connected to the normal ordering problem.

- Behr et al. [9] considered so-called pseudo-evolutionary differential equations which are defined as equations of Cauchy type, $D_{t} F(x, t)=\alpha O_{x} F(x, t), F(x, 0)=f(x)$. Here, $D_{t}$ is an operator playing the role of the derivative with respect to $t, \alpha$ is a constant, $O_{x}$ is an operator of differential or pseudo-differential nature. In the concrete examples considered, $O_{x}$ is a sum of two non-commuting operators (e.g., $O_{x}=-\left(\alpha x-\beta \partial_{x}\right)$ or $\left.O_{x}=-\left(\alpha x+\beta \partial_{x}^{2}\right)\right)$, and the exponential of $O_{x}$ is found using operational and operator ordering techniques (like, e.g., the Zassenhaus formula, or the Crofton-identity $\left.e^{\lambda \partial_{x}^{m}} f(x)=f\left(x+m \lambda \partial_{x}^{m-1}\right) e^{\lambda \partial_{x}^{m}}\right)$. If one considers for $O_{x}$ a differential operator of at most first order, $O_{x}=q(x) \partial_{x}+v(x)$, the integration of the associated one-parameter group $\left(U_{\lambda} f\right)(x)=\left(e^{\lambda\left(q(x) \partial_{x}+v(x)\right)} f\right)(x)$ has been considered many times in the literature, see the survey of Goodenough and Lavault [63] (where also the connection to Riordan arrays is discussed).

\section{References}

[1] A. Aboud, J.-P. Bultel, A. Chouria, J.-G. Luque and O. Mallet, Word Bell polynomials, Sém. Lothar. Combin. 75 (2017), Article B75h.

[2] L. Accardi and A. Boukas, On the characteristic function of random variables associated with boson Lie algebras, Commun. Stoch. Anal. 4 (2010), 493-504.

[3] L. Accardi and A. Boukas, Normally ordered disentanglement of multi-dimensional Schrödinger algebra exponentials, Commun. Stoch. Anal. 12 (2018), 283-328.

[4] J. Agapito, On symmetric polynomials with only real zeros and nonnegative $\gamma$-vectors, Linear Algebra Appl. 451 (2014), 260-289.

[5] J. Agapito, P. Petrullo, D. Senato and M.M. Torres, Eulerian polynomials via the Weyl algebra action, J. Algebraic Combin., to appear.

[6] F. Alayont and N. Krzywonos, Rook polynomials in three and higher dimensions, Involve 6 (2013), 35-52.

[7] A. Barghi, Stirling numbers of the first kind for graphs, Australas. J. Combin. 70 (2018), 253-268. 
[8] N. Behr, G. Dattoli and G.H.E. Duchamp, S. Licciardi and K.A. Penson, Operational methods in the study of Sobolev-Jacobi polynomials, Mathematics 7 (2019), Article 124.

[9] N. Behr, G. Dattoli and A. Lattanzi, Operator ordering and solution of pseudo-evolutionary equations, Axioms 8 (2019), Article 35.

[10] N. Behr, V. Danos and I. Garnier, Combinatorial conversion and moment bisimulation for stochastic rewriting systems, Log. Methods Comput. Sci. 16 (2020), 3:1-3:45.

[11] N. Behr, V. Danos, I. Garnier and T. Heindel, The algebras of graph rewriting, arXiv:1612.06240v1 [math$\mathrm{ph}], 2016$.

[12] N. Behr, G.H.E. Duchamp and K.A. Penson, Combinatorics of chemical reaction systems, arXiv:1712.06575v2 [math-ph], 2017.

[13] N. Behr and P. Sobociński, Rule algebras for adhesive categories, Log. Methods Comput. Sci. 16 (2020), $2: 1-2: 38$.

[14] H.B. Benaoum, $h$-Analogue of Newton's binomial formula, J. Phys. A. 31 (1998), L751-L754.

[15] H.B. Benaoum, (q,h)-Analogue of Newton's binomial formula, J. Phys. A. 32 (1999), 2037-2040.

[16] G. Benkart, S.A. Lopes and M. Ondrus, A parametric family of subalgebras of the Weyl algebra. II: Irreducible modules, Recent developments in algebraic and combinatorial aspects of representation theory. Contemp. Math. 602, Amer. Math. Soc., Providence, RI (2013), 73-98.

[17] G. Benkart, S.A. Lopes and M. Ondrus, A parametric family of subalgebras of the Weyl algebra. I: Structure and automorphisms, Trans. Am. Math. Soc. 367 (2015), 1993-2021.

[18] W.M. Bent-Usman, A.M. Dibagulun, M.M. Mangontarum and C.B. Montero, An alternative q-analogue of the Rucinski-Voigt numbers, Commun. Korean Math. Soc. 33 (2018), 1055-1073.

[19] F. Bergeron and C. Reutenauer, Une interprétation combinatoire des puissances d'un opérateur différentiel linéaire, Ann. Sci. Math. Québec 11 (1987), 269-278.

[20] P. Blasiak, G.H.E. Duchamp and K.A. Penson, Combinatorics of second derivative: Graphical proof of Glaisher-Crofton identity, Adv. Math. Phys. (2018), Article 9575626.

[21] P. Blasiak, G.H.E. Duchamp, A.I. Solomon, A. Horzela and K.A. Penson, Combinatorial algebra for second-quantized quantum theory, Adv. Theor. Math. Phys. 14 (2010), 1209-1243.

[22] P. Blasiak and P. Flajolet, Combinatorial models of creation-annihilation, Sém. Lothar. Combin. 65 (2011), Article B65c.

[23] I.E. Bousbaa, A. Chouria and J.-G. Luque, A combinatorial Hopf algebra for the boson normal ordering problem, Ann. Inst. Henri Poincaré D 5 (2018), 61-102.

[24] K. Brádler, Hiking a generalized Dyck path: A tractable way of calculating multimode boson evolution operators, Comput. Phys. Commun. 196 (2015), 80-91.

[25] E. Briand, P.R.W. McNamara, R. Orellana and M. Rosas, Commutation and normal ordering for operators on symmetric functions, Sém. Lothar. Combin. 80 (2019), Article B80d.

[26] E. Briand, S.A. Lopes and M. Rosas, Normally ordered forms of powers of differential operators and their combinatorics, J. Pure Appl. Algebra 224 (2020), Article 106312.

[27] J.-P. Bultel, A Faà di Bruno subalgebra of the graded dual of word symmetric functions, Adv. in Appl. Math. 96 (2018), 334-359.

[28] D. Burde, On the matrix equation $X A-A X=X^{p}$, Linear Algebra Appl. 404 (2005), 147-165.

[29] S. Butler, F. Chung, J. Cummings and R. Graham, Juggling card sequences, J. Comb. 8 (2017), 507-539.

[30] R.R.S. Cantuba, Lie polynomials in q-deformed Heisenberg algebras, J. Algebra 522 (2019), 101-123.

[31] R.R.S. Cantuba, Lie polynomials in an algebra defined by a linearly twisted commutation relation, arXiv:1811.03843v1 [math.RA], 2018.

[32] R.R.S. Cantuba and M.A.C. Merciales, An extension of a q-deformed Heisenberg algebra and its Lie polynomials, Expo. Math., to appear. 
[33] R.R.S. Cantuba and S. Silvestrov, Torsion-type q-deformed Heisenberg algebra and its Lie polynomials, Silvestrov, Sergei (ed.) et al., Algebraic Structures and Applications. SPAS2017, Västerås and Stockholm, Sweden, October 4-6, 2017. Cham: Springer. Springer Proc. Math. Stat. 317 (2020), 575-592.

[34] R.R.S. Cantuba and S. Silvestrov, Lie polynomial characterization problems, Silvestrov, Sergei (ed.) et al., Algebraic Structures and Applications. SPAS2017, Västerås and Stockholm, Sweden, October 4-6, 2017. Cham: Springer. Springer Proc. Math. Stat. 317 (202), 593-601.

[35] R.O. Celeste, R.B. Corcino and K.J.M. Gonzales, Two approaches to normal order coefficients, J. Integer Seq. 20 (2017), Article 17.3.5.

[36] A.M. Chebotarev and T.V. Tlyachev, Normal forms, inner products, and Maslov indices of general multimode squeezings, Math. Notes 95 (2014), 721-737.

[37] A.M. Chebotarev, T.V. Tlyachev and A.A. Radionov, Generalized squeezed states and multimode factorization formula, Math. Notes 92 (2012), 700-713.

[38] P. Cheung and V. Kac, Quantum calculus, Springer, New York, 2002.

[39] W.S. Chung, The normal ordering procedure and coherent state of the Q-deformed generalized Heisenberg algebra, J. Gen. Lie Theory Appl. 8 (2014), Article 1000213.

[40] J. Cigler, Operatormethoden für q-Identitäten, Monatsh. Math. 88 (1979), 87-105.

[41] E. Clark and R. Ehrenborg, The excedance algebra, Discrete Math. 313 (2013), 1429-1435.

[42] P. Codara and O.M. D'Antona, Dyck-Eulerian digraphs, Electron. Notes Discrete Math. 68 (2018), 239244.

[43] P. Codara, O.M. D'Antona and P. Hell, A simple combinatorial interpretation of certain generalized Bell and Stirling numbers, Discrete Math. 318 (2014), 53-57.

[44] L. Cohen, The Weyl operator and its generalization, Birkhäuser/Springer Basel AG, Basel, 2013.

[45] L. Comtet, Une formule explicite pour les puissances successives de l'opérateur de dérivation de Lie, C. R. Acad. Sci. Paris Sér. A-B 276 (1973), A165-A168.

[46] R.B. Corcino, R.O. Celeste and K.J.M. Gonzales, Rook theoretic proofs of some identities related to Spivey's Bell number formula, Ars Combin. 132 (2017), 11-26.

[47] R.B. Corcino, J.M. Ontolan, J. Cañete and M.J.R. Latayada, A q-analogue of $r$-Whitney numbers of the second kind and its Hankel transform, J. Math. Computer Sci. 21 (2020), 258-272.

[48] S. Corteel, M. Josuat-Vergès and L.K. Williams, The Matrix Ansatz, orthogonal polynomials, and permutations, Adv. in Appl. Math. 46 (2011), 209-225.

[49] B. Duncan and R. Peele, Bell and Stirling numbers for graphs, J. Integer Seq. 12 (2009), Article 09.7.1.

[50] A. Dzhumadil'daev and D. Yeliussizov, Path decompositions of digraphs and their applications to Weyl algebra, Adv. in Appl. Math. 67 (2015), 36-54.

[51] A. Dzhumadil'daev and D. Yeliussizov, Walks, partitions, and normal ordering, Electron. J. Combin. 22 (2015), Article 4.10.

[52] R. Ehrenborg, A. Happ, D. Hedmark and C. Hettle, Box polynomials and the excedance matrix, arXiv:1708.09804v1 [math.CO], 2017.

[53] B.S. El-Desouky, Generalized Stirling numbers of the first kind: Modified approach, J. Pure Appl. Math. Adv. Appl. 5 (2011), 43-59.

[54] B.S. El-Desouky, N.A. El-Bedwehy, A. Mustafa, N.A. El-Desouky and F. M. Abdel Moneim, A new generalized family of numbers and some applications, Preprint, 2019.

[55] J. Engbers, D. Galvin and J. Hilyard, Combinatorially interpreting generalized Stirling numbers, European J. Combin. 43 (2015), 32-54.

[56] S.-P. Eu, T.-S. Fu, Y.-C. Liang and T.-L. Wong, On xD-generalizations of Stirling numbers and Lah numbers via graphs and rooks, Electron. J. Combin. 24 (2017), Article 2.9.

[57] D. Finkelshtein, Y. Kondratiev, E. Lytvynov and M.J. Oliveira, Stirling operators in spatial combinatorics, arXiv:2007.01175v4 [math.CO], 2020. 
[58] U. Frisch and R. Bourret, Parastochastics, J. Mathematical Phys. 11 (1970), 364-390.

[59] D. Galvin, Asymptotic normality of some graph sequences, Graphs Combin. 32 (2016), 639-647.

[60] D. Galvin and A. Pacurar, Total non-negativity of some combinatorial matrices, J. Combin. Theory Ser. A 172 (2020), Article 105179.

[61] J. Goldman and J. Haglund, Generalized rook polynomials, J. Combin. Theory Ser. A 91 (2000), 509-530.

[62] M. de Gosson, Born-Jordan quantization, Springer, Cham, 2016.

[63] S. Goodenough and C. Lavault, Overview of the Heisenberg-Weyl algebra and subsets of Riordan subgroups, Electron. J. Combin. 22 (2015), Article 4.16.

[64] G.-N. Han and S.-M. Ma, Derivatives, Eulerian polynomials and the g-indexes of Young tableaux, arXiv:2006.14064v1 [math.CO], 2020.

[65] L. Hellström and S. Silvestrov, Commuting elements in q-deformed Heisenberg algebras, World Scientific, River Edge, NJ, 2000.

[66] G. Hetyei, Meixner polynomials of the second kind and quantum algebras representing $\mathrm{su}(1,1)$, Proc. R. Soc. Lond., Ser. A., Math. Phys. Eng. Sci. 466 (2010), 1409-1428.

[67] A. Hodges and C.V. Sukumar, Bernoulli, Euler, permutations and quantum algebras, Proc. R. Soc. Lond., Ser. A, Math. Phys. Eng. Sci. 463 (2007), 2401-2414.

[68] A. Hosseini and M. Mohammadzadeh Karizaki, The non-commutative Newton's binomial formula in nonunital algebras and with a negative power, Boll. Unione Mat. Ital. 13 (2020), 39-48.

[69] L.C. Hsu and P.J.-S. Shiue, A unified approach to generalized Stirling numbers, Adv. Appl. Math. 20 (1998), 366-384.

[70] F.H. Jackson, A q-generalization of Abel's series, Rend. Circ. Mat. Palermo 29 (1910), 340-346.

[71] W.P. Johnson, q-Extensions of identities of Abel-Rothe type, Discrete Math. 159 (1996), 161-177.

[72] M. Josuat-Vergès, Rook placements in Young diagrams and permutation enumeration, Adv. in Appl. Math. 47 (2011), 1-22.

[73] M. Josuat-Vergès, Enumeration of snakes and cycle-alternating permutations, Australas. J. Combin. 60 (2014), 279-305.

[74] L. Kargin and M. Can, Harmonic number identities via polynomials with $r$-Lah coefficients, C. R. Math. Acad. Sci. Paris 358 (2020), 535-550.

[75] L. Kargin and B. Çekim, Higher order generalized geometric polynomials, Turkish J. Math. 42 (2018), $887-903$.

[76] L. Kargin and R.B. Corcino, Generalization of Mellin derivative and its applications, Integral Transforms Spec. Funct. 27 (2016), 620-631.

[77] J. Katriel, Combinatorial aspects of boson algebra, Lett. Nuovo Cimento 10 (1974), 565-567.

[78] J. Katriel, Bell numbers and coherent states, Phys. Lett. A 273 (2000), 159-161.

[79] J. Katriel, On a generalized recurrence for Bell numbers, J. Integer Seq. 11 (2008), Article 08.3.8.

[80] J. Katriel and M. Kibler, Normal ordering for deformed boson operators and operator-valued deformed Stirling numbers, J. Phys. A 25 (1992), 2683-2691.

[81] U.N. Katugampola, Mellin transforms of generalized fractional integrals and derivatives, Appl. Math. Comput. 257 (2015), 566-580.

[82] Z.R. Kereskényiné Balogh and M.J. Schlosser, q-Stirling numbers of the second kind and q-Bell numbers for graphs, Electron. Notes Discrete Math. 54 (2016), 361-366.

[83] D.E. Knuth and T.J. Buckholtz, Computation of tangent, Euler, and Bernoulli numbers, Math. Comp. 21 (1967), 663-688.

[84] P. Kuchment and S. Lvin, Paley-Wiener theorem for exponential Radon transform, Acta Appl. Math. 18 (1990), 251-260.

[85] P. Kuchment and S. Lvin, The range of the exponential Radon transform, Soviet Math. Dokl. 42 (1991), $183-184$. 
[86] P. Kuchment and S. Lvin, Identities for $\sin x$ that came from medical imaging, Amer. Math. Monthly 120 (2013), 609-621.

[87] P. Kuchment and S. Lvin, Some binomial formulas for non-commuting operators, Contemp. Math. 733 (2019), 197-207.

[88] G. Labelle and C. Lamathe, General combinatorial differential operators, Sém. Lothar. Combin. 61 (2009), Article B61Ag.

[89] M.A. Lohe and A. Thilagam, Weyl-ordered polynomials in fractional-dimensional quantum mechanics, J. Phys. A 38 (2005), 461-483.

[90] W.H. Louisell, Radiation and Noise in Quantum Electronics, McGraw-Hill Inc., US, 1964.

[91] M. Maltenfort, New definitions of the generalized Stirling numbers, Aequationes Math. 94 (2020), 169-200.

[92] M.M. Mangontarum, Some theorems and applications of the $(q, r)$-Whitney numbers, J. Integer Seq. 20 (2017), Article 17.2.5.

[93] M.M. Mangontarum, Spivey's Bell number formula revisited, J. Integer Seq. 21 (2018), Article 18.1.1.

[94] M.M. Mangontarum and A.M. Dibagulun, Some generalizations of Spivey's Bell number formula, Matimyás Mat. 40 (2017), 1-11.

[95] M.M. Mangontarum and J. Katriel, On q-boson operators and q-analogues of the $r$-Whitney and $r$-Dowling numbers, J. Integer Seq. 18 (2015), Article 15.9.8.

[96] T. Mansour, J.L. Ramírez and M. Shattuck, A generalization of the $r$-Whitney numbers of the second kind, J. Comb. 8 (2017), 29-55.

[97] T. Mansour and M. Schork, The commutation relation $x y=q y x+h f(y)$ and Newton's binomial formula, Ramanujan J. 25 (2011), 405-445.

[98] T. Mansour and M. Schork, Commutation relations, normal ordering, and Stirling numbers, CRC Press, Boca Raton, FL, 2016.

[99] T. Mansour and M. Schork, On a close relative of the quantum plane, Mediterr. J. Math. 15 (2018), Article 124.

[100] T. Mansour, M. Schork and M. Shattuck, On a new family of generalized Stirling and Bell numbers, Electron. J. Combin. 18 (2011), Article 77.

[101] T. Mansour, M. Schork and M. Shattuck, The generalized Stirling and Bell numbers revisited, J. Integer Seq. 15 (2012), Article 12.8.3.

[102] V.P. Maslov, Operational methods, Mir Publishers, Moscow, 1976.

[103] M.A. Méndez, Combinatorial differential operators in: Fà̀ di Bruno formula, enumeration of ballot paths, enriched rooted trees and increasing rooted trees, arXiv:1610.03602v1 [math.CO], 2016.

[104] M.A. Méndez and J.L. Ramírez, A new approach to the $r$-Whitney numbers by using combinatorial differential calculus, Acta Univ. Sapientiae Math. 11 (2019), 387-418.

[105] I. Mezö, A new formula for the Bernoulli polynomials, Results. Math. 58 (2010), 329-335.

[106] I. Mezö, Combinatorics and number theory of counting sequences, CRC Press, Boca Raton, FL, 2020.

[107] M. Mihoubi and M.S. Maamra, The $\left(r_{1}, \ldots, r_{p}\right)$-Stirling numbers of the second kind, Integers 12 (2012), Article A35.

[108] M. Mohammad-Noori, Some remarks about the derivation operator and generalized Stirling numbers, Ars Combin. 100 (2011), 177-192.

[109] J. Musonda, S. Kaijser and S. Silvestrov, Reordering, centralizers and centers in an algebra with three generators and Lie type relations, Silvestrov, Sergei (ed.) et al., Algebraic Structures and Applications. SPAS2017, Västerås and Stockholm, Sweden, October 4-6, 2017. Cham: Springer. Springer Proc. Math. Stat. 317 (2020) 491-508.

[110] J. Musonda, J. Richter and S. Silvestrov, Reordering in a multi-parametric family of algebras, J. Phys.: Conf. Ser. 1194 (2019), Article 012078. 
[111] J. Musonda, J. Richter and S. Silvestrov, Reordering in noncommutative algebras associated with iterated function systems, Silvestrov, Sergei (ed.) et al., Algebraic Structures and Applications. SPAS2017, Västerås and Stockholm, Sweden, October 4-6, 2017. Cham: Springer. Springer Proc. Math. Stat. 317 (2020), 509-552.

[112] J. Musonda, J. Richter and S. Silvestrov, Twisted difference operator representations of deformed Lie type commutation relations, Silvestrov, Sergei (ed.) et al., Algebraic Structures and Applications. SPAS2017, Västerås and Stockholm, Sweden, October 4-6, 2017. Cham: Springer. Springer Proc. Math. Stat. 317 (2020), 553-573.

[113] A. Navon, Combinatorics and fermion algebra, Nuovo Cimento B 16 (1973), 324-330.

[114] V.E. Nazaikinskii, V.E. Shatalov and B.Yu. Sternin, Methods of Noncommutative Analysis, Walter de Gruyter, Berlin, 1995.

[115] L. Oussi, $A(p, q)$-deformed recurrence for the Bell numbers, J. Integer Seq. 23 (2020), Art. 20.5.2.

[116] C. Pita-Ruiz, Generalized Stirling numbers I, arXiv:1803.05953v1 [math.CO], 2018.

[117] J.H. Przytycki, q-Polynomial invariant of rooted trees, Arnold Math. J. 2 (2016), 449-461.

[118] J.L. Ramírez and M. Shattuck, Generalized $r$-Whitney numbers of the first kind, Ann. Math. Inform. 46 (2016), 175-193.

[119] H. Rosengren, A non-commutative binomial formula, J. Geom. Phys. 32 (2000), 349-363.

[120] H. Scherk, De evolvenda functione $(y d \cdot y d \cdot y d \ldots y d X) / d x^{n}$ disquisitiones nonnullae analyticae, (Ph.D. Thesis), University of Berlin, 1823.

[121] M.J. Schlosser, A noncommutative weight-dependent generalization of the binomial theorem, Sém. Lothar. Combin. 81 (2020), Art. B81j.

[122] M.J. Schlosser and M. Yoo, Some combinatorial identities involving noncommuting variables (Proceedings of FPSAC 2015), Discrete Math. Theor. Comput. Sci. Proc. (2015), 961-972.

[123] M.J. Schlosser and M. Yoo, Weight-dependent commutation relations and combinatorial identities, Discrete Math. 341 (2018), 2308-2325.

[124] A. Schreiber, Inverse relations and reciprocity laws involving partial Bell polynomials and related extensions, Enumer. Combin. Appl. 1 (2021), Art. S2R3.

[125] A.V. Sills, A q-analog of the binomial distribution, arXiv:2009.12641v2 [math.PR], 2020.

[126] Z. Škoda, A note on symmetric orderings, Acta Mathematica Spalatensia 1 (2020), 53-60.

[127] N.J.A. Sloane, The On-line Encyclopedia of Integer Sequences, http://oeis.org/

[128] M.Z. Spivey, A generalized recurrence for Bell numbers, J. Integer Seq. 11 (2008), Art. 08.2.5.

[129] C.V. Sukumar and A. Hodges, Quantum algebras and parity-dependent spectra, Proc. R. Soc. Lond., Ser. A., Math. Phys. Eng. Sci. 463 (2007), 2415-2427.

[130] A.E. Teretenkov, Irreversible quantum evolution with quadratic generator: Review, Infin. Dimens. Anal. Quantum Probab. Relat. Top. 22 (2019), Art. 1930001.

[131] A. Varvak, Rook numbers and the normal ordering problem, J. Combin. Theory Ser. A 112 (2005), 292-307.

[132] O.V. Viskov, An approach to ordering, Dokl. Math. 57 (1998), 69-71.

[133] W. Wyss, Quasi-multiplication and $*$-algebras, Rep. Math. Phys. 18 (1980), 87-95.

[134] W. Wyss, Two non-commutative binomial theorems, arXiv:1707.03861v2 [math.RA], 2017.

[135] J. Xiong and Q. Fang, Differential operators and Narayana numbers, Open J. Discrete Appl. Math. 3 (2020), 37-40. 